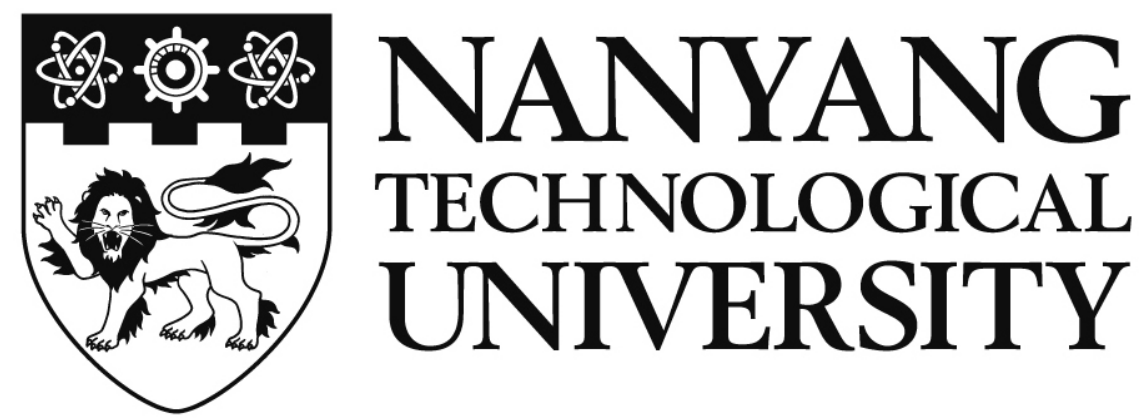

DISCOVERING CLASS-SPECIFIC VISUAL PATTERNS

FOR VISUAL RECOGNITION

WENG CHAOQUN

SCHOOL OF ELECTRICAL \& ELECTRONIC

ENGINEERING

2016 



\section{DISCOVERING CLASS-SPECIFIC VISUAL PATTERNS FOR VISUAL RECOGNITION}

\section{WENG CHAOQUN}

School of Electrical \& Electronic Engineering

A thesis submitted to the Nanyang Technological University in partial fulfillment of the requirement for the degree of Doctor of Philosophy

2016 

To my family 


\section{Acknowledgements}

First and foremost, I would like to express my sincerely gratitude to my supervisor, Professor Junsong Yuan, who has directed me into such a beautiful and fantastic research realm of computer vision and machine learning, and supported me with well-equipped environment and active research atmosphere. Prof. Yuan has supported me throughout my Ph.D. study with his patience and knowledge, and encouraged me to explore my own way of research. During these years of studying under his supervision, I have learned a lot. I have been greatly impressed by his professional attitude towards research and teaching.

I also wish to thank all the members in our research group, Chunluan Zhou, Du Tran, Gangqiang Zhao, Gang Yu, Hongxing Wang, Hui Liang, Jianfeng Ren, Jingjing Meng, Kang Dang, Yang Cong, Yang Xiao, Ye Luo, Yuning Jiang, and Zhou Ren, among many other kind people who have helped me and taught me a lot during group meetings and daily life.

Finally, my gratitude goes to my dear family and all the friends in my life! 



\section{Table of Contents}

Acknowledgements $\quad$ i

Table of Contents iii

$\begin{array}{lll}\text { Abstract } & \text { vii }\end{array}$

List of Figures $\quad$ ix

List of Tables $\quad$ xi

1 Introduction 1

1.1 Motivations and Objectives . . . . . . . . . . . . . . . . . . . 1

1.2 Main Contributions of the Thesis . . . . . . . . . . . . . . 5

1.2.1 Spatial and Feature Context Aware Pattern Discovery . . . . . . . . . 5

1.2.2 Spatial Layout Pattern Discovery ............. . . . 5

1.2.3 AND/OR Co-occurrence Pattern Mining . . . . . . . . . . . . 6

1.3 Organization of the Thesis . . . . . . . . . . . . . . 7

2 Discovering Spatial Context Aware and Multi-feature Fused Visual Phrase Patterns 9

2.1 Introduction . . . . . . . . . . . . . . . . . 9

2.2 Related Work . . . . . . . . . . . . . . . . . . . . 11

2.3 Proposed Method . . . . . . . . . . . . . . . . . . . 13

2.3.1 Learning Spatial Context Aware Visual Phrases . . . . . . . . . . . . . . . . 13

2.3.1.1 Visual Primitive Sparse Coding . . . . . . . . . . . . . . 13

2.3.1.2 Visual Primitive Spatial Pooling . . . . . . . . . . . . . . . 15

2.3.1.3 Visual Phrase Sparse Coding . . . . . . . . . . . . . . 16

2.3.1.4 Visual Phrase Codebook Learning . . . . . . . . . . . . 17

2.3.1.5 Back Propagation . . . . . . . . . . . . . . 17

2.3.1.6 Visual Primitive Codebook Learning . . . . . . . . . . . . . 18

2.3.1.7 Summarization . . . . . . . . . . . . . . . . . . . 19

2.3.2 Learning Multi-feature Fused Visual Phrases . . . . . . . . . . . . . . . 20

2.3.3 Learning Discriminative Visual Phrases . . . . . . . . . . . . . . . 21

2.4 Experiments . . . . . . . . . . . . . . . . . . 25

2.4.1 Texture Pattern Discovery . . . . . . . . . . . . . . 25

2.4 .2 Scene Clustering . . . . . . . . . . . . . . . 26 
2.4.2.1 Comparing Multi-feature Fusion Results . . . . . . . . . . . 28

2.4.2.2 Comparing Visual Phrase Learning Results . . . . . . . . . . 29

2.4.3 Scene Categorization on Scene-15 Dataset . . . . . . . . . . . . . . 31

2.5 Conclusion and Future Work . . . . . . . . . . . . . . . . . . 32

3 Discovering Class-specific Spatial Layout Patterns for Scene Recognition 35

3.1 Introduction . . . . . . . . . . . . . . . . 35

3.2 Proposed Method . . . . . . . . . . . . . . . . 38

3.2 .1 Feature Extraction . . . . . . . . . . . . . . . 38

3.2 .2 Proposed Method . . . . . . . . . . . . . . . . . 39

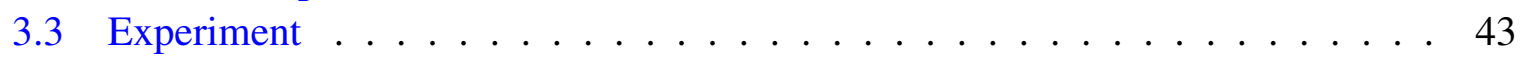

3.3.1 Scene-15 Dataset . . . . . . . . . . . . . . . . 43

3.3.2 Landuse-21 Dataset . . . . . . . . . . . . . . . . . . . . 45

3.3.3 MIT Indoor-67 Dataset . . . . . . . . . . . . . . . . . . . 46

3.4 Conclusion and Future Work . . . . . . . . . . . . . . . . . . 48

4 Efficient Discovery of AND/OR Co-occurrence Patterns for Visual Recognition 51

4.1 Introduction . . . . . . . . . . . . . . . . . . . 51

4.2 Related Work . . . . . . . . . . . . . . . . . . . 53

4.3 Proposed Method . . . . . . . . . . . . . . . . . 55

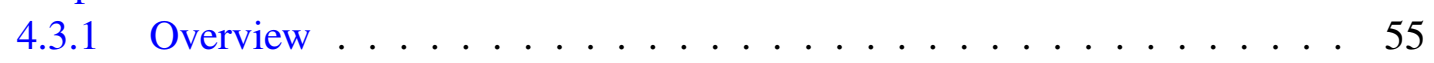

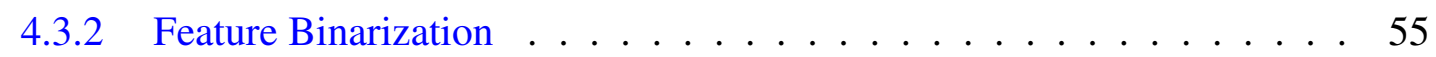

4.3 .3 Induced Transaction $\ldots \ldots \ldots \ldots 57$

4.3.4 Mining Optimal AND/OR Patterns . . . . . . . . . . . . . . 57

4.3.5 Efficiently Mining Optimal AND/OR Patterns . . . . . . . . . . . . . . 59

4.3.5.1 Pattern Frequency Constraints . . . . . . . . . . . . . . 59

4.3.5.2 Branch-and-Bound Search . . . . . . . . . . . . . 61

4.3.6 Boosting with Mined Optimal AND/OR Patterns . . . . . . . . . . . . 64

4.4 Experiments . . . . . . . . . . . . . . . . . 65

4.4.1 Benchmark Datasets . . . . . . . . . . . . . 67

4.4.1.1 Effectiveness Test . . . . . . . . . . . . 67

4.4.1.2 Efficiency Test . . . . . . . . . . . . . . . 68

4.4 .2 Scene Recognition Dataset . . . . . . . . . . . . . . . . . . . 69

4.4.3 Action Recognition Dataset . . . . . . . . . . . . . . . . 75

4.5 Conclusion and Future Work . . . . . . . . . . . . . . . . . 77

5 Learning Attentional AND/OR Skeleton Patterns for 3D Action Recognition 79

5.1 Introduction . . . . . . . . . . . . . . . . . 79

5.2 Related Work . . . . . . . . . . . . . . . . . . . . . . 82

5.3 Proposed Method . . . . . . . . . . . . . . . . . 84

5.3.1 Skeleton Features . . . . . . . . . . . . . . . . . . 84

$5.3 .2 \quad$ AND/OR Pattern Mining . . . . . . . . . . . . . . . 85

5.3.2.1 Pattern Mining Overview . . . . . . . . . . . 85

5.3.2.2 Information Gain for Pattern Mining . . . . . . . . . . . 87

5.3.2.3 Information Gain Upper Bound . . . . . . . . . . . . . 89 
5.3.2.4 Branch and Bound Search . . . . . . . . . . . . . . . 90

5.3.3 Attention Model for Skeleton Joints and Skeleton Patterns . . . . . . . 90

5.3.3.1 Temporal Modeling with LSTM . . . . . . . . . . . . 91

5.3.3.2 Attention LSTM . . . . . . . . . . . . . . . . 92

5.4 Experiments . . . . . . . . . . . . . . . . . . . . 94

5.4.1 MSR Action 3D Dataset . . . . . . . . . . . . . . . . . . . . . 94

5.4 .2 MSR Daily Activity 3D Dataset . . . . . . . . . . . . . . . 96

5.4 .3 SBU Interaction Dataset . . . . . . . . . . . . . . . . . 97

5.4 NTU RGBD Dataset . . . . . . . . . . . . . . . . . . . . . . . . . . . . . . . . . . . . . . . . . .

5.4.5 Conclusion and Future Work . . . . . . . . . . . . . . 100

6 Conclusion and Future Work $\quad 103$

6.1 Conclusion . . . . . . . . . . . . . . . . . . 103

6.2 Future Work . . . . . . . . . . . . . . . . . . . . . 104

6.2.1 Discovering Visual Patterns from Convolution Neural Networks . . . . 104

6.2.2 Discovering Class-specific Temporal Layout Patterns for Action Recognition . . . . . . . . . . . . . . . . . 105

6.2.3 Learning Attention LSTM Model with Tree Structure . . . . . . . . . . 105

$\begin{array}{ll}\text { Author's Publications } & 107\end{array}$

$\begin{array}{lr}\text { Bibliography } & 109\end{array}$ 



\section{Abstract}

Similar to frequent patterns in data mining, visual pattern refers to a recurring composition of visual contents in images or videos, such as repetitive texture regions, common objects among images, or similar actions among videos. Such visual patterns capture the recurrence nature of visual data and can represent the essence of the visual data. Finding such visual patterns is critical to image and video data analysis.

In spite of the recent successes of unsupervised mining of representative visual patterns in unlabeled visual data, for visual recognition tasks, the unsupervised mined visual patterns are often not discriminative enough to distinguish among different classes. One natural way to overcome this limitation is to leverage supervised learning and discover class-specific visual patterns, which is the focus of this thesis. Particularly, we target at discovering the following visual patterns of different structures: (1) class-specific local spatial patterns, e.g., local texture structure that can help differentiate different object images; (2) class-specific spatial layout patterns, e.g., spatial grid patterns that can help differentiate different scene images; (3) classspecific visual pattern of compositional structures, e.g., conjunction (AND) and disjunction (OR) forms of individual visual features that can help differentiate different scene images and action videos.

To discover the above-mentioned class-specific visual patterns, this thesis is composed by the following technical works. In the first work, we propose to mine mid-level visual phrases from low-level visual primitives, e.g., local image patches or regions, by leveraging local spatial context of visual primitives, multi-feature fusion of visual primitives, and also the weaklysupervised image label information. In the second work, we propose to discover class-specific spatial layouts for each scene category by casting a $\ell_{1}$-regularized max-margin optimization problem. In the third work, we propose a novel branch-and-bound based co-occurrence pattern mining algorithm that can directly mine both optimal conjunctions (AND) and disjunctions (OR) of individual features at arbitrary orders simultaneously with minimum classification error 
for boosting algorithm. Similar to the third work, in the fourth work we aim to discover highorder AND/OR patterns of skeleton features from depth camera for action recognition. We also propose to integrate the discovered AND/OR patterns in an attention LSTM model for temporal modeling to improve action recognition performance.

Compared with unsupervised visual pattern discovery, which usually separates the step of pattern discovery and classification, our method can provide a joint learning of visual pattern discovery and visual recognition. Also, different from conventional visual recognition which emphasize purely on the classification performance, our class-specific visual patterns target more on capturing the essence of difference visual classes, such that we not only can recognize the visual classes, but also can explain and understand why they are different visual classes, thanks to the discovered class-specific visual patterns. 


\section{List of Figures}

1.1 Samples of versatile visual patterns. Top left shows an LV bag image with visual patterns of repetitive texture logos. Top right shows the different global spatial layout patterns of "street" and "coast" scene images. Bottom left sketches the "cat" objects with visual patterns of two ears, two eyes, one nose, one mouth, two claws and so on. Bottom right shows the "playing tennis" action with visual patterns of a person, a tennis racket and the human body posture to

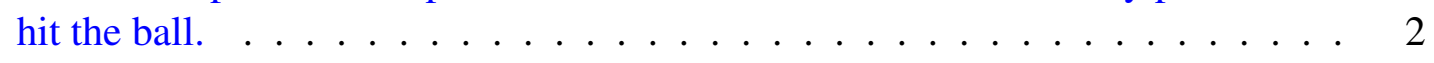

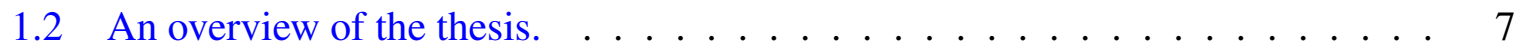

2.1 The proposed discriminative visual phrase learning algorithm via spatial context aware multi-feature fusion sparse coding, in which the main variables to be optimized are the classifier weights matrix $W$, the visual phrase codebook $U$ and sparse codes $V$, and the visual primitive codebook $B$ and sparse codes $C$. 14

2.2 Illustration of spatial pooling and multi-feature fusion. . . . . . . . . . . 16

2.3 Illustration of an LV bag image. In the left we show the original image; In the middle we show the visual patterns contained in the original image; In the right we show the colors used for the visual primitives (e.g., SIFT points detected by [1]) located at different visual patterns. . . . . . . . . . . . . . . . . . . 28

2.4 Sample images from MSRC-V2 dataset [2] . . . . . . . . . . . . . . . . 30

2.5 Illustration of different types of features used to distinguish different region

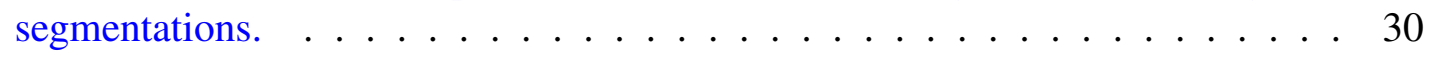

3.1 Illustration of the spatial layouts for street and coast scene categories. Comparing the $1^{\text {st }}$ row to the $2^{\text {nd }}$ and $3^{\text {rd }}$ rows, we can see that street and coast categories have different spatial layouts due to large inter-class variations. From the $2^{\text {nd }}$ and $3^{\text {rd }}$ rows, we can see that within even the same category, images can also exhibit different spatial layouts due to large intra-class variations. . . . . . . . . 36

3.2 Illustration of the feature extraction pipeline for the proposed method. (a) shows the input image. (b) shows the 2D feature maps of the convolution and pooling layers of the convnets. (c) shows the multiple random partitions to for$\mathrm{m}$ different spatial layouts. (d) shows the extracted features using the fc layer for different partitions. (e) shows the stacked data matrix for the input image. From the figure we can see that, we insert a random partitioning layer between the convolution/pooling layers and the fc layers of the convnets, and finally output a stacked data matrix as the data representation for each image. . . . . . 40

3.3 Confusion matrix for Scene-15 dataset. . . . . . . . . . . . . . . . . . 45

3.4 Random partition coefficients for Scene-15 dataset. . . . . . . . . . . . . . 46 
3.5 Confusion matrix for Landuse-21 dataset. . . . . . . . . . . . . . . . . . . . 47

4.1 Overview of the proposed efficiently mining optimal AND/OR patterns for boosting method. ...................... 56

4.2 Illustration of the induced transaction using random projected decision stumps on $2 \mathrm{D}$ points. . . . . . . . . . . . . . . . . . . 58

4.3 Learning curve comparisons between boosting with decision stumps and our boosting with AND/OR patterns. . . . . . . . . . . . . . . 72

4.4 Running time comparisons between the two-round two-stage pattern mining method [3] and our branch-and-bound method. . . . . . . . . . . . 73

4.5 The confusion matrix of the Scene-15 dataset. . . . . . . . . . . . . . . . 75

4.6 The confusion matrix of MSR Daily Activity 3D dataset. . . . . . . . . . 78

5.1 Illustration of high order patterns of skeleton joints in spatial domain and moving visual attentions in temporal domain for action category "high arm waving". In the figure, we can see two spatial co-occurrence patterns, one formed by left shoulder and left elbow showed in light blue circle, the other containing left wrist and left hand showed in deep blue circle, moving in time dimension. Since the other skeleton joints are almost stationary, it is clear that visual attentions in temporal dimension will follow the movements of the two spatial patterns for action category "high arm waving". . . . . . . . . . . . . 81

5.2 Illustration of the AND/OR pattern mining pipeline. . . . . . . . . . . . 87

5.3 Illustration of attention LSTM model with joint features and high order AND/OR pattern features. In the figure, $X_{i}$ and $X^{\prime}{ }_{i}$ are the skeleton joint and skeleton pattern features for the $i^{\text {th }}$ frame respectively, $\hat{y}_{i}$ is the predicted action label, $\ell_{i}$ and $\ell_{i}^{\prime}$ are the predicted attention of skeleton joint and skeleton pattern respectively. . . . . . . . . . . . . . . . . .

5.4 Running time comparisons between proposed branch and bound search and exhaustive search of high order AND/OR patterns. . . . . . . . . . .

5.5 Illustration of discovered high order skeleton patterns (in conjunction form) composed by skeleton joints. In the figure, red dots represent raw skeleton joints and green dots represent the selected skeleton joints that form the high order skeleton patterns. (1) and (2) are from action "drink", (3) and (4) are from action "eat", (5)(6) are from action "walk". . . . . . . . . . .

5.6 Illustration of selected visual attentions of high order skeleton patterns for action "cheer up" in 5 frames. We select two skeleton patterns for each frame, based on top two highest coefficients as used in Eq. 5.33, and plot them in green circles. We can see the selected visual attentions over time are quite stable, although there is a change from frame (2) to frame (3). . . . . . . . 


\section{List of Tables}

2.1 Clustering results on the LV bag image by (a) sparse coding algorithm in Eq. 2.1; (b) our proposed VPL-SC method. . . . . . . . . . . . . . . . . 27

2.2 Clustering results on the MSRC-V2 dataset. . . . . . . . . . . . . . . . 30

2.3 Accuracy results on the Scene-15 dataset. . . . . . . . . . . . . . . . . 32

3.1 Accuracy results on the Scene-15 dataset. . . . . . . . . . . . . . . . 44

3.2 Accuracy results on the Landuse-21 dataset. . . . . . . . . . . . . . . . . . . . . . . . . . . . 47

3.3 Accuracy results on the MIT Indoor-67 dataset. . . . . . . . . . . . . . . 48

4.1 Benchmark datasets information. . . . . . . . . . . . . . 70

4.2 Accuracy results of the benchmark datasets. . . . . . . . . . . . . . 70

4.3 Accuracy results of the Scene-15 dataset. . . . . . . . . . . . . . . . . . 74

4.4 The number of discovered patterns of different orders on the Scene-15 dataset. . 75

4.5 The number of discovered patterns of different orders on the MSR Daily Activity $3 \mathrm{D}$ dataset. . . . . . . . . . . . . . . . . 76

4.6 Accuracy results of the MSR Daily Activity 3D dataset. . . . . . . . . . . . . 77

5.1 Accuracy results on MSR Action 3D dataset. . . . . . . . . . . . . . . . . . 96

5.2 Accuracy results of MSR Daily Activity 3D dataset. . . . . . . . . . . . . . . . . . . . . . . . . . . . . .

5.3 Accuracy results on SBU Interaction dataset. . . . . . . . . . . . . . . 99

5.4 Accuracy results on NTU-RGBD dataset. . . . . . . . . . . . . . . . 100 



\section{Chapter 1}

\section{Introduction}

\subsection{Motivations and Objectives}

Similar to frequent patterns in data mining, visual pattern refers to a recurring composition of visual contents in images or videos, such as repetitive texture regions, common objects among images, or similar actions among videos. Such visual patterns capture the recurrence nature of visual data and can represent the essence of the visual data. Finding such visual patterns is critical to image and video data analysis. For example, as shown in Fig. 1.1, visual patterns can be (a) repetitive image textons, e.g., the texture logos from an LV bag image; (b) compositional object models with multiple distinguished visual parts, e.g., the "cat" objects with two ears, two eyes, one nose, one mouth, two claws and so on; (c) spatial scene layout patterns from different scene categories; (d) sequences of frames capturing human actions, e.g., the "playing tennis" actions including one person, one tennis racket and the human posture to hit the ball. Mining representative and semantic visual patterns from large amounts of image and video data can help us understand the data well and thus plays a critical role for effective visual recognition.

The idea of visual pattern discovery from unlabeled dataset has been exploited in different domains such as image matching [4], common pattern discovery [5-7], object recognition [8], action recognition [9-11]. For visual recognition tasks, the unsupervised mined visual patterns 

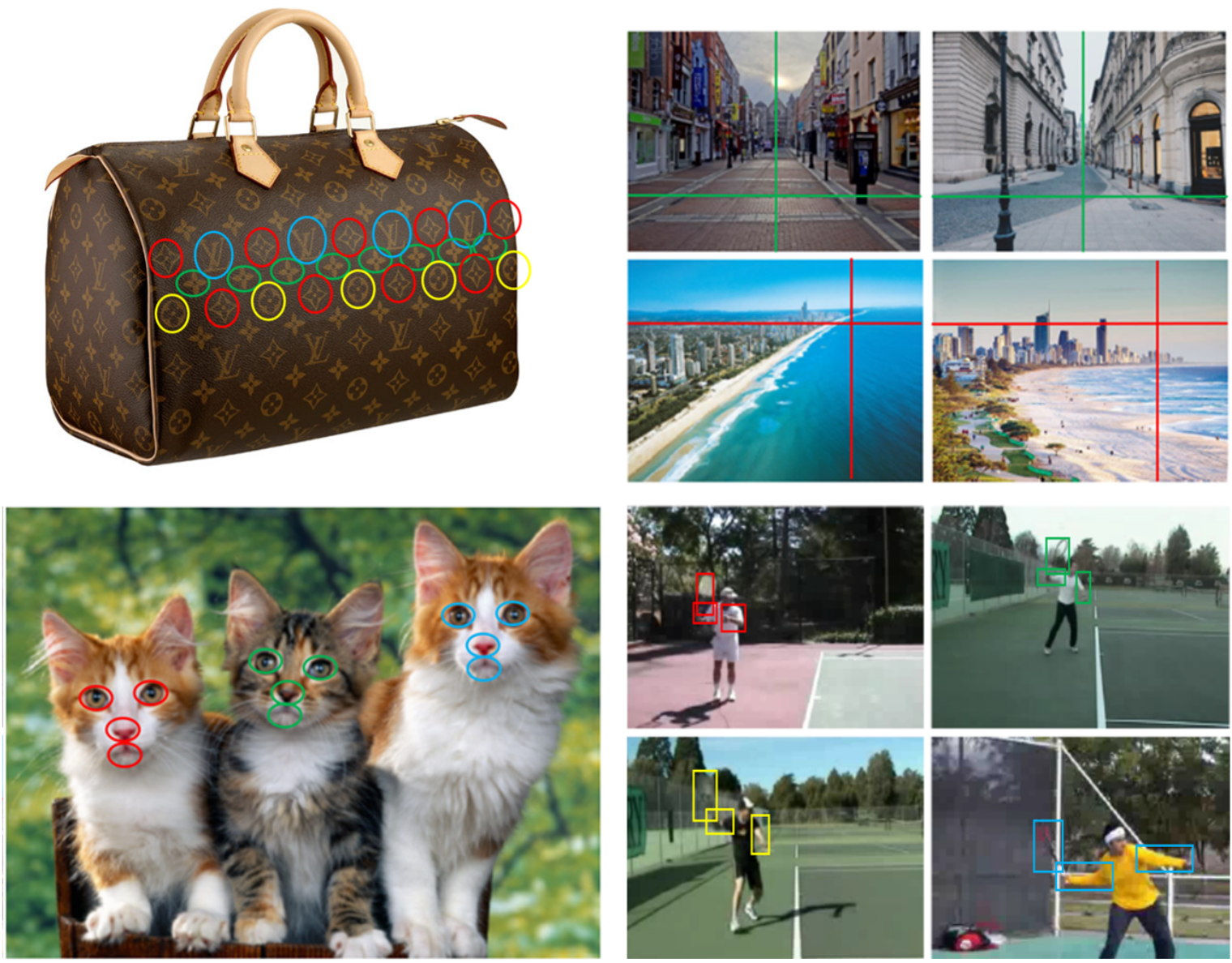

FIGURE 1.1. Samples of versatile visual patterns. Top left shows an LV bag image with visual patterns of repetitive texture logos. Top right shows the different global spatial layout patterns of "street" and "coast" scene images. Bottom left sketches the "cat" objects with visual patterns of two ears, two eyes, one nose, one mouth, two claws and so on. Bottom right shows the "playing tennis" action with visual patterns of a person, a tennis racket and the human body posture to hit the ball.

are often not discriminative enough to distinguish among different classes. One natural way to overcome this limitation is to integrate supervised learning and visual pattern discovery. It is of great interests to integrate supervised learning and visual pattern discovery in a joint framework, however supervised visual pattern discovery still remains a challenging problem due to the following challenges.

First of all, image and video data are usually represented by high-dimensional feature vectors. Due to the large variation of high-dimensional features, it is not a trivial task to discover meaningful visual patterns from images and videos. The appearance variations greatly challenge most existing frequent pattern mining algorithms [12-15] developed for text mining. 
Second, visual data contain rich spatial structure information, such as the spatial dependency of local visual primitives and the class-specific spatial layouts of the whole image, which is essential for understanding visual data [3, 16-18]. Without exploiting the complex spatial structure within visual data, there is no guarantee the conventional pattern mining algorithms for text mining can work for visual recognition effectively.

Third, image and video data can be characterized by multiple types of visual features, e.g., color feature, shape feature and motion feature. Instead of using an individual feature, it is of great interests to discover visual patterns among multiple feature modalities for effective visual recognition. In spite of the great successes of many previous works on unsupervised multi-feature fusion [19-21], it still remains a challenging problem to efficiently mine the discriminative visual patterns of multiple feature modalities from labeled data. What's more, the importance of conjunction (AND) and disjunction (OR) forms of individual features are often ignored for image and video data analysis. How to efficiently discover discriminative AND/OR patterns and combine them into an ensemble classifier is also of great interests.

Fourth, visual scene is a composition of foreground objects and background contexts, and due to the large inter-class and intra-class variations of spatial layouts of scene images, it is worth exploring how to discover discriminative class-specific spatial layout patterns for scene recognition.

From the above observations, in this thesis, we focus on discovering the following visual patterns: (1) class-specific local spatial patterns, e.g., local texture patterns that can help differentiate different visual classes; (2) class-specific global spatial layout patterns, e.g., spatial grid patterns that can help distinguish different visual scenes; (3) class-specific visual pattern of compositional structures, e.g., conjunction (AND) and disjunction (OR) forms of individual features that can help differentiate different classes of scene images and action videos.

To discover the above mentioned class-specific visual patterns, this thesis is composed by four technical works.

In the first work, we propose to mine mid-level visual phrases from low-level visual primitives, 
e.g., local image patches or regions, by leveraging local spatial context of visual primitives, multi-feature structure of visual primitives, and also the weakly-supervised image label information. Experiments show that our proposed algorithm can learn more representative and discriminative visual phrases for visual recognition tasks, such as texture pattern discovery, scene clustering and object recognition.

In the second work, we propose to discover class-specific spatial layouts for scene recognition by casting a max-margin optimization problem. Unlike previous methods that either use classgeneric spatial layouts or use pre-defined spatial layouts, our joint learning of class-specific spatial layouts and image classifier can achieve superior performance for scene recognition problem, by leveraging the recent deep learning features.

In the third work, we propose a novel branch-and-bound based co-occurrence pattern mining algorithm that can directly mine both optimal conjunctions (AND) and disjunctions (OR) of individual features at arbitrary orders simultaneously. This pattern mining process is integrated into boosting framework such that the weighted error is minimized by the discovered co-occurrence pattern in each boosting step. Experiments on versatile benchmark datasets show that our proposed algorithm achieves superior performances than algorithms using raw individual features directly.

Similar to the third work, in the fourth work we aim to discover high order AND/OR patterns of skeleton features from depth camera for action recognition. We propose to efficiently mine the optimal AND/OR patterns of skeleton features with maximum information gain given labeled multi-class transaction dataset. We also propose to combine the discovered AND/OR patterns in an attention long-short term memory (LSTM) model to improve the performance of action recognition.

Compared with unsupervised visual pattern discovery, which usually separates the step of pattern discovery and classification, our method can provide a joint learning of visual pattern discovery and visual recognition. Also, different from conventional visual recognition which emphasizes purely on the classification performance, our class-specific visual patterns target more on capturing the essence of difference visual classes, such that we not only can recognize 
the visual classes, but also can explain and understand why they are different visual classes, thanks to the discovered class-specific visual patterns.

\subsection{Main Contributions of the Thesis}

\subsubsection{Spatial and Feature Context Aware Pattern Discovery}

Due to the semantic gap [18] between low-level local features, i.e., visual primitives, and higher level concepts, i.e., visual patterns, it is a challenging task to mine visual patterns without considering the spatial dependency among visual primitives.

Besides, visual primitives are often represented by multiple types of visual features, e.g., color feature, shape feature etc., it is difficult to group them into visual patterns without considering multi-feature fusion. For example, on the one hand, two visual primitives that share similar visual features, e.g., color feature, can belong to different visual patterns, e.g., blue sky and blue ocean; On the other hand, two visual primitives that exhibit dissimilar visual features, e.g., color feature, can be of the same visual patterns, e.g., green apple and red apple.

In Chapter 2, we propose to mine mid-level visual phrases from local visual primitives by leveraging the local spatial neighborhood context of visual primitives, multi-feature fusion of visual primitives and also the weakly-supervised image label information.

\subsubsection{Spatial Layout Pattern Discovery}

Instead of adopting an unsupervised manner to use either use class-generic spatial layouts or pre-defined spatial layouts for image recognition, which would lead to suboptimal recognition performance [22-24], in Chapter 3 we propose to explore the global spatial layout structure of scene images and discover class-specific spatial layout patterns for each scene category from the labeled scene dataset for image classification. 
In order to discover class-specific spatial layouts for visual recognition, first we represent each image by a set of feature vectors under different spatial layout configurations, then we propose a max-margin framework with $\ell_{1}$-norm regularization to jointly select discriminative spatial layouts and learn the image classifier from labeled scene dataset. To solve the proposed maxmargin objective function, we apply alternating optimization method to learn the coefficients of both spatial layouts and image classifiers.

\subsubsection{AND/OR Co-occurrence Pattern Mining}

Despite the great successes of previous frequent pattern mining algorithms $[12,13]$ in data mining community, there are still many limitations and challenges.

First of all, conventional data mining algorithms only mine frequent patterns, however these frequent patterns may not be the optimal choices for visual recognition tasks such as object/scene recognition and action recognition. Second, traditional frequent pattern mining algorithms are very time-consuming especially when the input features are of large dimensions. Third, after the co-occurrence patterns are discovered, how to integrate them appropriately into an effective classifier also needs to be studied.

In Chapter 4 and 5, we address the above mentioned challenges. Instead of mining frequent patterns, we mine the optimal co-occurrence pattern with minimum classification error or maximum information gain. Instead of the exhaustive search for frequent patterns, we propose a branch-and-bound search method that can efficiently discover the optimal co-occurrence pattern. In Chapter 4 we also use boosting algorithm to combine the discovered co-occurrence patterns to achieve better generalization error for multi-class classification problems. In Chapter 5 we also propose to apply an attention LSTM model to combine the discovered AND/OR patterns. 


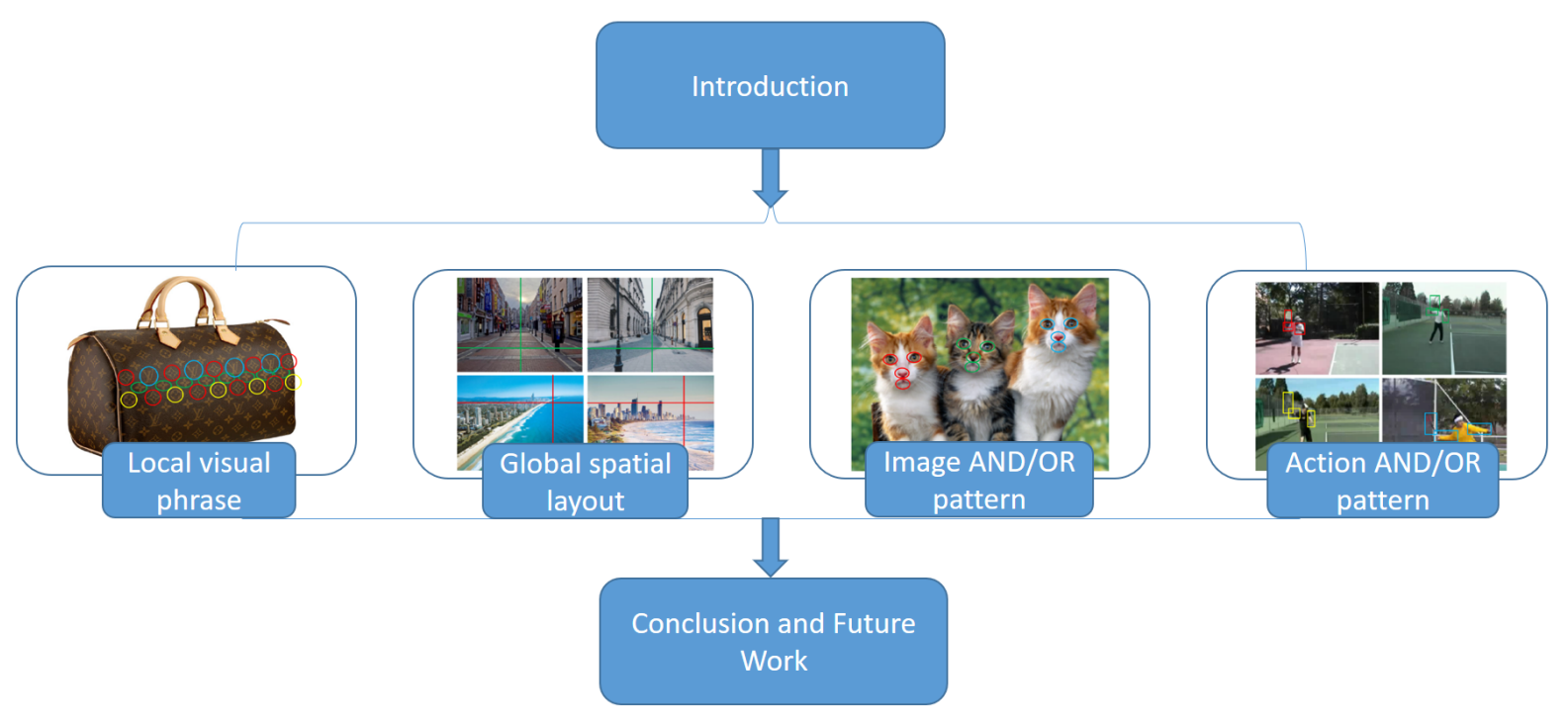

FIGURE 1.2. An overview of the thesis.

\subsection{Organization of the Thesis}

The rest of this thesis is organized as follows, which can also be found from Fig. 1.2.

In Chapter 2 and 3 we discover visual patterns by exploiting spatial context information. To be specific, in Chapter 2, we propose to mine mid-level visual phrases from low-level visual primitives, e.g., local image patches or regions. In this work, both spatial context information and multi-feature representation information for low-level local visual primitives are exploited to discover mid-level visual phrases. In this chapter, we focus on discovering local visual phrases for various computer vision applications, such as texture pattern discovery, image clustering. In Chapter 3, we aim to discover class-specific global spatial layouts of scene images for scene recognition. Different from Chapter 2, in this work we focus on discovering global spatial layout patterns by exploiting the image label information for scene recognition.

In Chapter 4 and 5 we discover visual patterns by exploiting multi-feature co-occurrence information. To be specific, in Chapter 4, we propose to mine feature combinations, i.e., high-order AND/OR co-occurrence patterns, to improve image recognition performance. In this work, we focus on efficiently mining AND/OR patterns from raw images with multi-feature representations for image recognition. In Chapter 5, we propose to mine high-order AND/OR 
co-occurrence patterns from raw skeleton points for action recognition. After the high order AND/OR skeleton patterns in spatial domain are discovered, we also propose to learn visual attentions of skeleton patterns via attention LSTM model in temporal domain. Different from Chapter 4, in this work we focus on action recognition based on raw 3D skeleton data, so we not only consider to mining the spatial AND/OR skeleton patterns, but also consider learning the temporal attentions of skeleton patterns via attention LSTM model.

In Chapter 6, we have conclusions of our works and also describe some potential future works. 


\section{Chapter 2}

\section{Discovering Spatial Context Aware and Multi-feature Fused Visual Phrase}

\section{Patterns}

\subsection{Introduction}

The development of visual recognition applications, such as object recognition, object detection and scene understanding, need descriptive and discriminative representations of images, such as [23, 25, 49, 51-54]. For example, CNN features such as AlexNet [51], VGG network [52], ResNet [54] have achieved impressive image classification performances on ILSVRC imagenet dataset. Different from CNN features, the bag-of-words (BoW) model [25] is also one of the popular algorithms for solving visual recognition problems. In the BoW model, local descriptors such as SIFT [1] are extracted from images, upon which a visual vocabulary is trained using k-means algorithm. After that, the SIFT descriptors are quantized into visual words using the visual vocabulary. Finally all the visual words are pooled into a histogram to form a global feature for the original image.

Despite its simplicity, the BoW model is lack of spatial context of low-level visual words which can lead to less effective recognition performance. More representative mid-level visual phrase 
(e.g., image patterns) features can be learnt by incorporating spatial context information upon low-level visual primitive (e.g., local image patches or salient regions) features used in the BoW model, such as [17, 26-31]. For example, the work in [26, 30] used data mining approaches to discover higher-level spatial co-occurrent visual phrase patterns upon lower-level visual words and improved the performance of traditional BoW method. The work in [17] applied geometrypreserving visual phrases to encode spatial information into BoW representations for image retrieval. Besides investigating spatial co-occurrence of low-level visual words in the BoW method, the work in [29] also tried to discover meaningful chunks which are bigger than objects and smaller than scenes as visual phrase features for visual recognition.

However there still exist many limitations in previous visual phrase learning works in spite of their great successes. First, previous works often encoded local visual primitive descriptors into visual words by hard quantization and counted the co-occurrence of visual words to form visual phrases. Due to the quantization error of visual primitives, it can lead to less representative visual phrases using spatial co-occurrence of visual words from the unlabeled dataset. Second, previous works often focused on single type of local descriptors such as the SIFT descriptors. Since visual primitives can be described by different types of features, e.g., color, shape and texture, it can be beneficial to fuse multiple features that provide more descriptive information from various aspects. Third, previous works did not incorporate the image label information in the visual phrase learning process and thus would result in less discriminative visual phrases.

In this chapter, we propose to learn discriminative mid-level visual phrase sparse codes by incorporating spatial context information and multi-feature evidences of low-level visual primitives, and exploring the image label information. Given multiple types of low-level visual primitive features, we first learn their sparse codes respectively by conventional sparse coding algorithms. Then we cast these visual primitive sparse codes of local spatial neighborhood into mid-level visual phrases by spatial pooling. Along with the spatial pooling in the spatial space, we also fuse the multiple types of visual primitive sparse codes by feature concatenation in the feature space. After that, we further learn the sparse codes for the visual phrases which can be more representative compared with the low-level visual primitive sparse codes. In order 
to obtain discriminative sparse representations for visual phrases, we also propose to jointly train a classifier and optimize the codebooks for both visual primitives and visual phrases via back-propagation. The proposed back-propagation optimization guarantees to converge, and leads to more discriminative and also multi-feature fused visual phrases for visual recognition. Since we have explored the spatial context information, multi-feature evidences fusion and the image label information, more representative and discriminative visual phrase sparse codes can be obtained. The experiments on image pattern discovery, image scene clustering, scene categorization and object recognition justify the advantages of the proposed algorithm.

The remainder of the chapter is organized as follows. In Sec. 2.2 we have a literature review of the related work. In Sec. 2.3 we will introduce our discriminative visual phrase learning algorithm based on spatial context aware multi-feature fusion sparse coding. In Sec. 2.4 we conduct experiments on the image pattern discovery, image scene clustering, scene categorization problems. In Sec. 2.5 we have a conclusion.

\subsection{Related Work}

Based on low-level visual primitive descriptors [1], the most popular visual recognition algorithms using visual primitive codebooks have been introduced in the past dozen years, such as $[16,23,25,32,33]$.

Although the unsupervised codebook training methods, such as k-means and Gaussian Mixture Model, have already shown their popularity in many applications, researchers have also tried incorporating the image label information to train more discriminative codebooks, as described in recent works [34-39].

Numerous works on learning more representative mid-level visual phrase features by incorporating spatial context information upon the low-level visual primitive features have been proposed, such as [17, 27-31, 40-43]. For example, the work in [40] proposed to discover image patterns by encoding spatial context of SIFT descriptors in the k-means framework. Later 
it was extended in [42] by considering both spatial context and multi-feature fusion. Mid-level visual phrases are also popularly used in image retrieval applications to enhance the BoW model by adding the spatial co-occurrence of low-level visual words and have achieved impressive retrieval performances $[17,30,31,44]$.

Since images can be described by different types of features, such as bag-of-words feature, color histogram, it will be beneficial if we can effectively fuse the multiple independent or heterogeneous features. Previous research works also have demonstrated that superior performance can be obtained for visual recognition problems if we properly utilize the multi-feature evidences, compared with algorithms that use single type of feature or just concatenate different features as one feature, such as [42, 45-48]. For example, the work in [42] introduced a regularized term, which contains the concatenations of hard quantization codes of different features, to the original k-means algorithm for clustering. In the work [45], researchers trained a multiple kernel learning algorithm to utilize the different features for object recognition. The work in [47] proposed to train a uniform codebook by putting different types of local descriptors together, which requires the local descriptors are all of the same dimension.

In terms of exploring both spatial context information and multi-feature evidences fusion of low-level visual primitives, the work in [42] adopted the similar idea as ours to discover midlevel visual phrases. However the work in [42] can only learn the hard quantization codes from the unsupervised k-means algorithm, while our proposed algorithm can learn more representative and discriminative sparse codes for visual phrases from the labeled image dataset and achieve superior performance.

More recently, different from the coding of low-level visual primitive descriptors, feature learning from image pixels using hierarchical architectures have gained more and more attentions in the area of computer vision due to its significant performance improvement, such as [49-56]. For example, AlexNet [51] achieved the $1^{\text {st }}$ place with top- 5 test error rate $15.3 \%$ compared to the $26.2 \%$ achieved by the $2^{\text {nd }}$ best method on the ILSVRC 2012 challenge. The VGG16 network [52] has secured the $1^{\text {st }}$ and the $2^{\text {nd }}$ places in the localization and classification tracks respectively on ImageNet Challenge 2014. The ResNet [54] achieved 3.57\% error on the 
ImageNet test set and won the $1^{\text {st }}$ place on the ILSVRC 2015 classification task.

\subsection{Proposed Method}

Let us declare the notations in this section first. We use upper case letter to denote a matrix, e.g., $X$. We then use upper case letter with two subscripts (row index and column index respectively) to denote an element of the matrix, e.g., $X_{i j}$. We use lower case letter with one subscript to denote each column of the matrix, e.g., $x_{i}$.

\subsubsection{Learning Spatial Context Aware Visual Phrases}

In this section we introduce the mid-level visual phrase discovering method by considering the spatial neighborhood of low-level visual primitives. As shown in Fig. 2.1, we construct the mid-level visual phrases, e.g., image patterns, from low-level visual primitives, e.g., local image patches or regions, of a local spatial neighborhood. We follow the traditional descriptorcoding-pooling pipeline in the first layer to encode local visual primitives. Then in the second layer we try to discover the spatial context aware and also multi-feature fused visual phrases. We use sparse coding method to encode visual phrases such that we can then create global image representations based on these sparse coded visual phrases. Compared with individual visual primitives, visual phrases have a more complex structure and convey richer information thus can be more discriminative.

In the following we will discuss our spatial context aware visual phrase learning algorithm step by step as shown in Fig. 2.1.

\subsubsection{Visual Primitive Sparse Coding}

We start from the visual primitive sparse coding for images. An image is represented by a set of local descriptors $D=\left[d_{1}, d_{2}, \cdots, d_{M}\right] \in \mathbb{R}^{P \times M}$ where each column vector $d_{i}$ represents a 


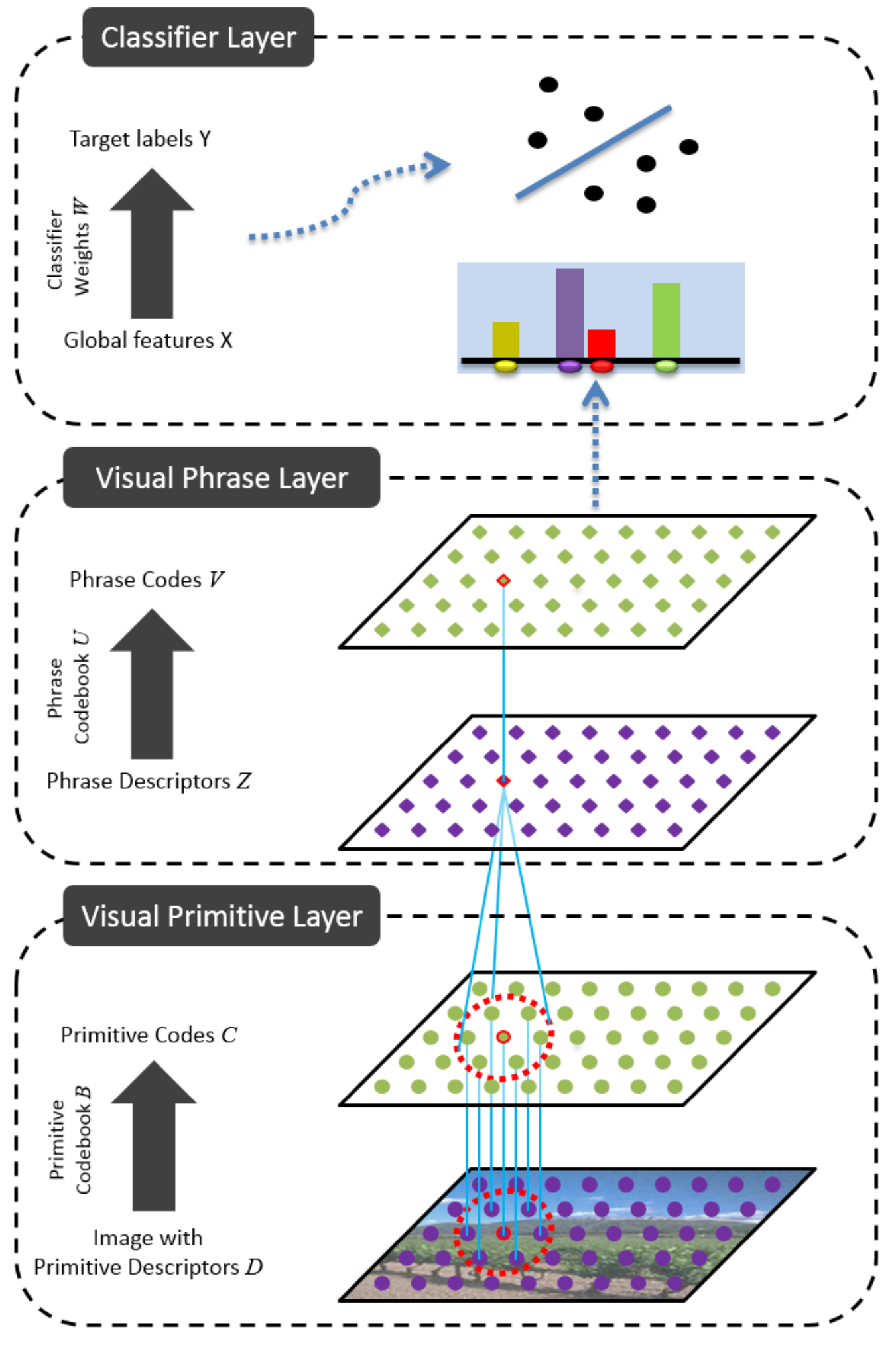

FIGURE 2.1. The proposed discriminative visual phrase learning algorithm via spatial context aware multi-feature fusion sparse coding, in which the main variables to be optimized are the classifier weights matrix $W$, the visual phrase codebook $U$ and sparse codes $V$, and the visual primitive codebook $B$ and sparse codes $C$. 
visual primitive, e.g., a local image patch or region. Given a codebook $B \in \mathbb{R}^{P \times K_{1}}$ where $K_{1}$ is the codebook size, the sparse coding representation $C \in \mathbb{R}^{K_{1} \times M}$ of the descriptor set $D$ can be calculated as follows:

$$
\hat{C}=\arg \min _{C} J_{1}=\arg \min _{C}\|D-B C\|_{\ell_{2}}^{2}+\lambda_{1}\|C\|_{\ell_{1}}
$$

where $\|\cdot\|_{\ell_{2}}$ is the Frobenius norm of a matrix and $\|\cdot\|_{\ell_{1}}$ is the $\ell_{1}$ norm.

\subsubsection{Visual Primitive Spatial Pooling}

In order to incorporate the spatial context information of the low-level visual primitives, we pool the sparse codes of the visual primitives in a local spatial neighborhood by $k-N N$ or $\epsilon-N N$. The spatial pooling process is illustrated in Fig. 2.2.

We consider two commonly used spatial pooling methods, average pooling [25] and max pooling [23]. Assume that $z_{j}$ is the $j^{\text {th }}$ spatial pooled visual phrase and $c_{i}$ is the sparse codes of the local descriptor $d_{i}$, then average pooling is shown as follows:

$$
z_{j}=\sum_{i \in S(j)} \frac{1}{|S(j)|} c_{i}
$$

where $S(j)$ denotes the set of local descriptors contained in the $j^{\text {th }}$ visual phrase.

The max pooling is shown as follows:

$$
z_{j}=\max _{i \in S(j)}\left(c_{i}\right)
$$

where the max operation is the element-wise max operation.

As discussed in [27], max pooling method tends to produce more discriminative representations when soft coding methods are used, while average pooling method on the contrary, works better when hard quantization method is applied. 


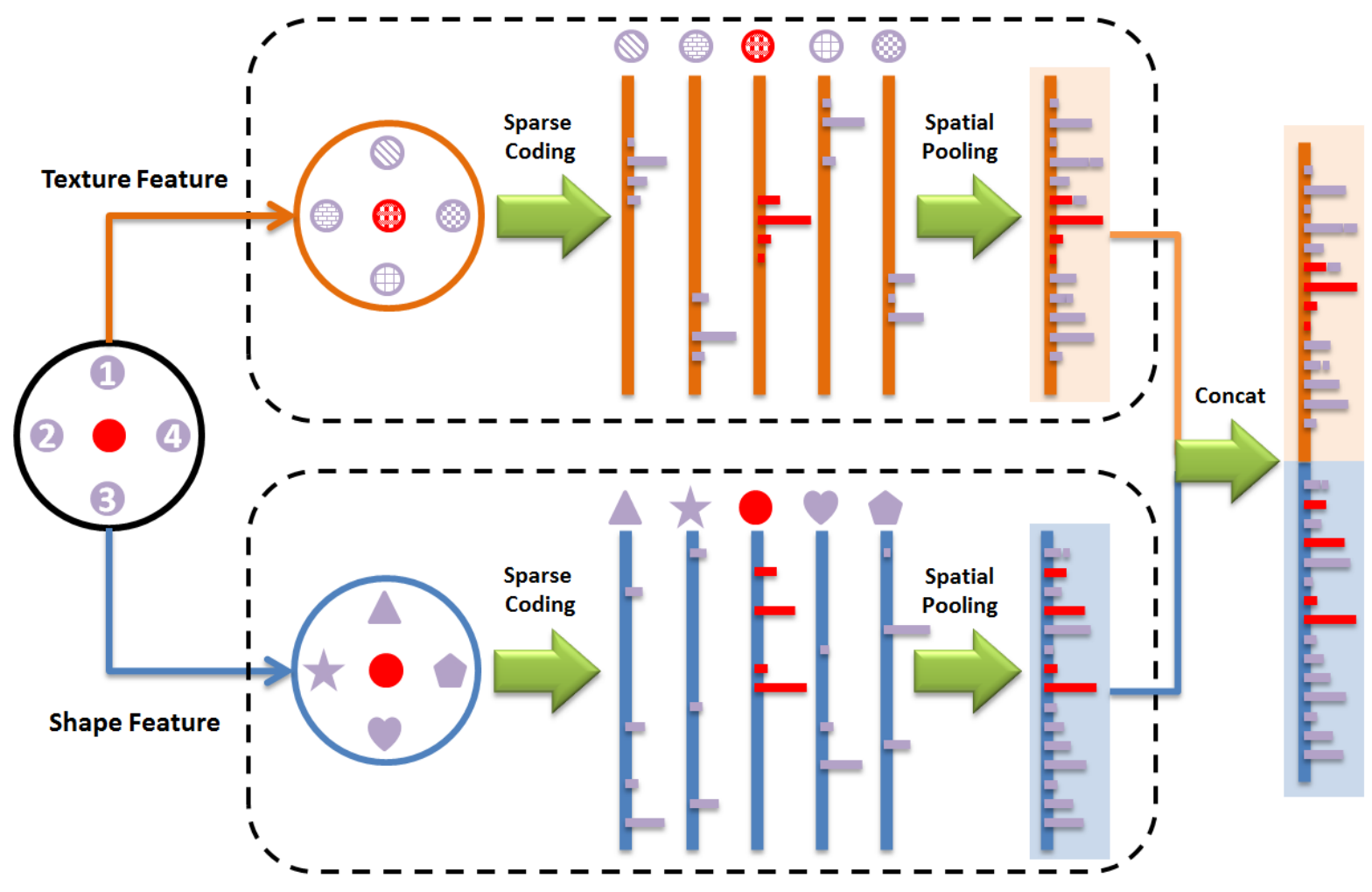

FIGURE 2.2. Illustration of spatial pooling and multi-feature fusion.

\subsubsection{Visual Phrase Sparse Coding}

After the spatial pooling, we have obtained the visual phrase descriptor set $Z=\left[z_{1}, z_{2}, \cdots, z_{M}\right] \in$ $\mathbb{R}^{K_{1} \times M}$ where each column $z_{j}$ is a feature vector to describe the $j^{\text {th }}$ visual phrase. It is worth noting that the visual phrase features, i.e., the spatial pooled visual primitive sparse codes in a local spatial neighborhood, are still local features. In order to build bag-of-visual-phrases image representation that contains spatial context information than traditional bag-of-visualwords representation, we also apply sparse coding for the visual phrases. Similarly we can calculate the sparse codes $V \in \mathbb{R}^{K_{2} \times M}$ of the descriptor set $Z$ by Eq. 2.4,

$$
\hat{V}=\arg \min _{V} J_{2}=\arg \min _{V}\|Z-U V\|_{\ell_{2}}^{2}+\lambda_{2}\|V\|_{\ell_{1}}
$$

where $U \in \mathbb{R}^{K_{1} \times K_{2}}$ is the given visual phrase codebook and $K_{2}$ is the codebook size.

With the help of the visual phrase sparse coding step, we can jointly optimize the sparse codes and codebooks of visual primitives and visual phrases via back propagation and thus obtain 
more representative visual phrases. The benefits of the sparse coding of visual phrases will be further explored in the experiments.

\subsubsection{Visual Phrase Codebook Learning}

As our target is to learn representative visual phrase sparse codes which require high-quality codebooks, we now describe how to optimize the two codebooks $B$ and $U$ for visual primitive and visual phrase features respectively. To optimize the visual phrase codebook $U$, we fix all other variables in Eq. 2.4 except for $U$ and then solve Eq. 2.5 as discussed in [57],

$$
\begin{array}{r}
\hat{U}=\arg \min _{U}\|Z-U V\|_{\ell_{2}}^{2} \\
\text { s.t. }\left\|u_{i}\right\|_{\ell_{2}}^{2} \leq 1, \quad \forall i=1, \cdots, K_{2} .
\end{array}
$$

\subsubsection{Back Propagation}

In order to optimize the visual primitive codebook $B$, however we have to compute the gradient of $J_{2}$ with respect to $B$ using the chain rule as follows:

$$
\frac{\partial J_{2}}{\partial B}=\sum_{j} \frac{\partial J_{2}}{\partial z_{j}} \times\left(\sum_{i} \frac{\partial z_{j}}{\partial c_{i}} \times \frac{\partial c_{i}}{\partial B}\right)
$$

From Eq. 2.4 we can easily compute $\frac{\partial J_{2}}{\partial z_{j}}$, as shown in Eq. 2.7,

$$
\frac{\partial J_{2}}{\partial z_{j}}=2\left(z_{j}-U v_{j}\right)
$$

According to the different spatial pooling methods applied in Sec. 2.3.1.2, we have different back propagation strategies. When the average pooling method in Eq. 2.2 is used, Eq. 2.6 becomes:

$$
\frac{\partial J_{2}}{\partial B}=\sum_{j} \frac{\partial J_{2}}{\partial z_{j}} \times\left(\sum_{i \in S(j)} \frac{1}{|S(j)|} \times \frac{\partial c_{i}}{\partial B}\right)
$$


When the max pooling method in Eq. 2.3 is applied, Eq. 2.6 becomes:

$$
\frac{\partial J_{2}}{\partial B}=\sum_{j} \frac{\partial J_{2}}{\partial z_{j}} \times \operatorname{sign}\left(y_{i}^{\max }\right) \odot \frac{\partial c_{i}^{\max }}{\partial B}
$$

where $\odot$ is the element-wise product symbol and $c_{i}^{\max }$ is obtained as follows:

$$
c_{i}^{\max }=\max _{i \in S(j)}\left(c_{i}\right)
$$

Therefore in order to compute the derivative $\frac{\partial J_{2}}{\partial B}$ in Eq. 2.6 we now focus on $\frac{\partial c_{i}}{\partial B}$, which will be discussed in the next section.

\subsubsection{Visual Primitive Codebook Learning}

According to the above discussions, in order to optimize the visual primitive codebook $B$, we have to compute $\frac{\partial c_{i}}{\partial B}$. Since $c_{i}$ is not directly linked to $B$ according to Eq. 2.1, we have to compute $\frac{\partial c_{i}}{\partial B}$ by the implicit differentiation method.

First we calculate the gradient with respect to $c_{i}$ at its minimum $\hat{c}_{i}$ for Eq.2.1, as used in [58],

$$
\left.2\left(B^{T} B c_{i}-B^{T} d_{i}\right)\right|_{c_{i}=\hat{c_{i}}}=-\lambda_{1} \times\left.\operatorname{sign}\left(c_{i}\right)\right|_{c_{i}=\hat{c_{i}}}
$$

It is worth noting that Eq. 2.11 is only correct when $c_{i}=\hat{c}_{i}$. For convenience, in the following we will admit the condition that $c_{i}=\hat{c}_{i}$ without explicitly showing it in the equations.

Then we calculate the gradient with respect to $B$ on both sides of Eq. 2.11 and get,

$$
\frac{\partial\left\{2\left(B^{T} B c_{i}-B^{T} d_{i}\right)\right\}}{\partial B_{m n}}=\frac{\partial\left\{-\lambda_{1} \times \operatorname{sign}\left(c_{i}\right)\right\}}{\partial B_{m n}}
$$

where $B_{m n}$ is the $m^{\text {th }}$ row and $n^{\text {th }}$ column element of the codebook $B$.

Note that the right-hand side of Eq. 2.12 is not well-defined at zero due to the non-continuous property of $\operatorname{sign}\left(c_{i}\right)$, therefore we choose the non-zero coefficients from $\hat{c}_{i}$ to form $\tilde{c}_{i}$ and select 
the corresponding codebook bases $\tilde{B}$ by $\tilde{c}_{i}$, and get the following result:

$$
\frac{\partial\left\{2\left(\tilde{B}^{T} \tilde{B} \tilde{c}_{i}-\tilde{B}^{T} d_{i}\right)\right\}}{\partial B_{m n}}=0
$$

By expanding Eq. 2.13, we can further get:

$$
\tilde{B}^{T} \tilde{B} \frac{\partial \tilde{c}_{i}}{\partial B_{m n}}+\frac{\partial \tilde{B}^{T} \tilde{B}}{\partial B_{m n}} \tilde{c}_{i}-\frac{\partial \tilde{B}^{T} d_{i}}{\partial B_{m n}}=0
$$

which leads to the final result of $\frac{\partial c_{i}}{\partial B}$ :

$$
\frac{\partial \tilde{c}_{i}}{\partial B_{m n}}=\left(\tilde{B}^{T} \tilde{B}\right)^{-1}\left(\frac{\partial \tilde{B}^{T} d_{i}}{\partial B_{m n}}-\frac{\partial \tilde{B}^{T} \tilde{B}}{\partial B_{m n}} \tilde{c}_{i}\right)
$$

In practise, due to the sparse solution of $c_{i}$, the selected $\tilde{B}$ has much less number of bases than the descriptor dimension. Therefore $\left(\tilde{B}^{T} \tilde{B}\right)^{-1}$ can be well-conditioned.

\subsubsection{Summarization}

To summarize our spatial context aware visual phrase learning algorithm, we combine the previously discussed sparse coding and codebook learning steps for both the visual primitive features and the visual phrase features and show the proposed VPL-SC algorithm in Alg. 1. It is worth noting that our target is to learn representative visual phrase sparse codes $V$, therefore we need to update codebooks $B$ and $U$ via back-propagation. Once the codebooks $B$ and $U$ are updated, the corresponding sparse codes $C$ and $V$ can be computed according to Eq. 2.1 and Eq. 2.4. In the experiments, when given codebooks we use the SPAMS toolbox ${ }^{1}$ to compute the sparse codes. To update the codebooks in our proposed algorithms, we use back-propagation optimization and stochastic gradient descent method similar to the optimization of a neural network. From Eq. 2.15 we can see that the complexity of computing the gradient is $O\left(n^{3}\right)$, which lies on the computation of the inverse matrix of $\tilde{B}^{T} \tilde{B}$. The proposed back-propagation

\footnotetext{
${ }^{1}$ SPAMS toolbox. http://spams-devel.gforge.inria.fr/
} 


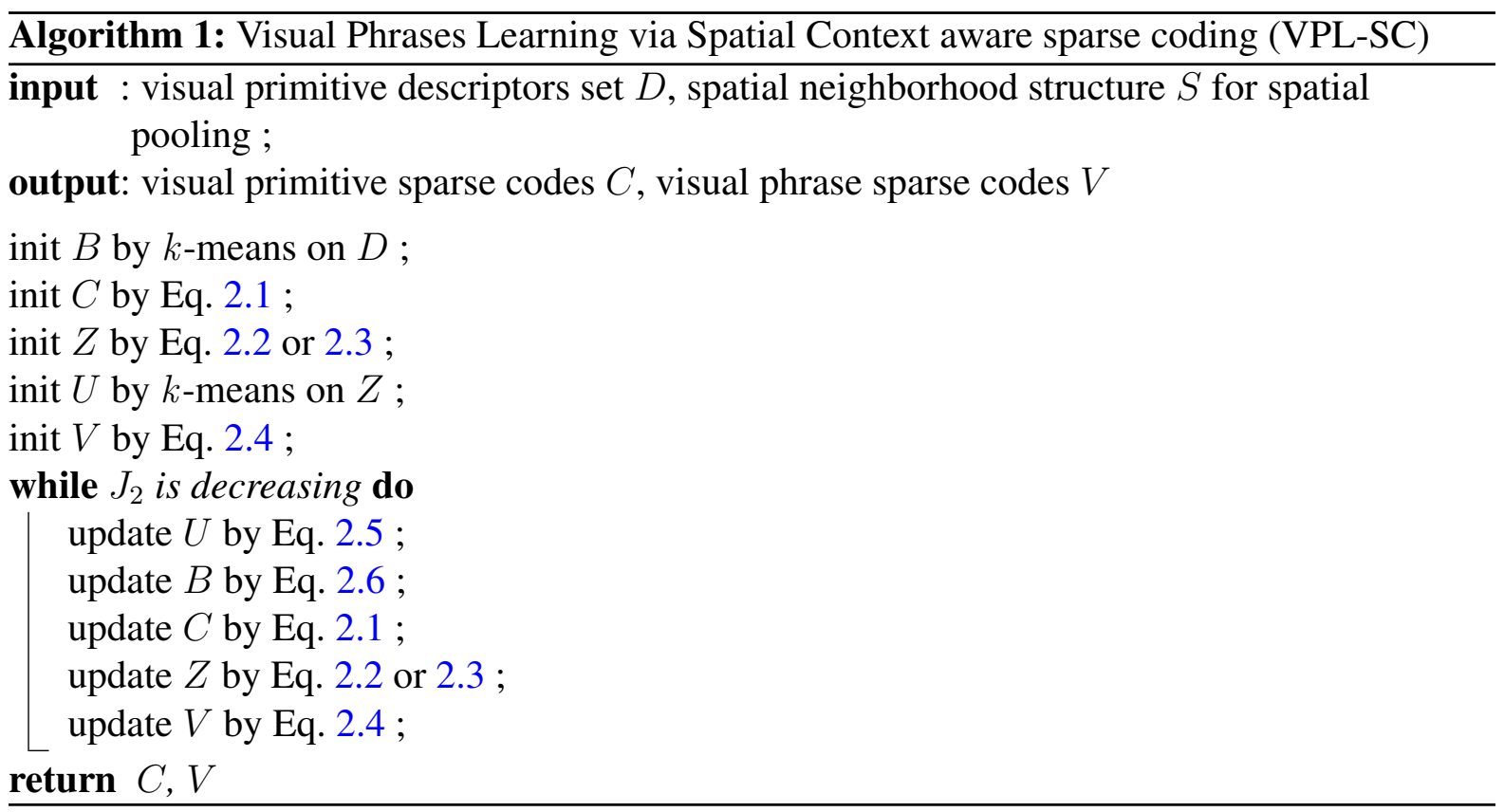

optimization guarantees to converge to a local optima. In our experiments, the objective usually converges after 10-20 iterations.

\subsubsection{Learning Multi-feature Fused Visual Phrases}

In the above section, we have discussed our mid-level visual phrase learning algorithm with single type of low-level visual primitives. Now let us consider fusing different types of visual primitives together to obtain more descriptive visual phrase features.

Assume that we have $\mathcal{T}$ types of different visual primitive descriptor sets $D=\left\{D^{(1)}, D^{(2)}, \cdots D^{(\mathcal{T})}\right\}$. For each descriptor set $D^{(t)}$, we can get the corresponding codebook $B^{(t)}$, the sparse codes $C^{(t)}$ by Eq. 2.1 and the spatial pooled representations $Z^{(t)}$ by average pooling in Eq. 2.2 or max pooling in Eq. 2.3. After that, we can concatenate all the $Z^{(t)}$ as follows,

$$
z_{i}=\bigcup_{t=1}^{\mathcal{T}}\left[z_{i}^{(t)}\right]
$$

where $\bigcup[\cdot]$ is the vector concatenation operator. 
After the concatenation, the new descriptor set $Z$ for the visual phrases contains both spatial context information and multi-feature evidences, which can be more descriptive than previous algorithm that only used single type of visual primitive feature.

In order to update each $B^{(t)}$ and $Y^{(t)}$, the back propagation Eq. 2.6 becomes,

$$
\frac{\partial J}{\partial B^{(t)}}=\sum_{j} \frac{\partial J}{\partial z_{j}^{(t)}} \times\left(\sum_{i} \frac{\partial z_{j}^{(t)}}{\partial c_{i}^{(t)}} \times \frac{\partial c_{i}^{(t)}}{\partial B^{(t)}}\right)
$$

where each $\frac{\partial J}{\partial z_{j}^{(t)}}$ is the $t_{t h}$ corresponding part in $\frac{\partial J}{\partial z_{j}}$.

Although multi-feature fusion can be exploited as early fusion by concatenating visual primitive descriptors, we propose to concatenate visual primitive sparse codes. This is due to the consideration that, early fusion method forces different visual primitive descriptor sets to share one common codebook, which can be problematic if different visual primitives have different codebook sizes. For example, the codebook size of color histogram features can differ from the codebook size of shape features. On the contrary, our method allows training different codebooks for different visual primitive descriptor sets and then fusing different sparse codes for the visual phrases to share multi-feature evidences. The benefits of fusing sparse codes will be further explored in the experiments.

The above discussed multi-feature fusion process is illustrated in Fig. 2.2. The proposed spatial context aware and multi-feature fused visual phrase learning algorithm VPL-SC-MF is presented in Alg. 2.

\subsubsection{Learning Discriminative Visual Phrases}

So far we have discussed the unsupervised learning of visual phrase sparse codes by incorporating both spatial context information and multi-feature evidences fusion, however for specific tasks such as scene categorization or object recognition applications, we can use the image label information to leverage the visual phrase learning process. 
Algorithm 2: Visual Phrases Learning via Spatial Context aware Multi-feature Fusion sparse coding (VPL-SC-MF)

input : visual primitive descriptors sets $\left\{D^{(t)}\right\}$, spatial neighborhood structure $S$ for spatial pooling ;

output: visual primitive sparse codes $\left\{C^{(t)}\right\}$, visual phrase sparse codes $V$;

for $t=1: \mathcal{T}$ do

init $B^{(t)}$ by $k$-means on $D^{(t)}$;

init $C^{(t)}$ by Eq. 2.1 ;

init $Z^{(t)}$ by Eq. 2.2 or 2.3 ;

init $Z$ by Eq. 2.16 ;

init $U$ by $k$-means on $Z$;

init $V$ by Eq. 2.4 ;

while $J_{2}$ is decreasing do

update $U$ by Eq. 2.5 ;

for $t=1: T$ do

update $B^{(t)}$ by Eq. 2.17 ;

update $C^{(t)}$ by Eq. 2.1 ;

update $Z^{(t)}$ by Eq. 2.2 or 2.3 ;

update $Z$ by Eq. 2.16 ;

update $V$ by Eq. 2.4 ;

return $\left\{C^{(t)}\right\}, V$

Upon the visual phrase sparse codes, we now add a classifier to guide the learning process of the visual phrase sparse codes, as shown in Fig. 2.1. From the local visual phrase sparse codes $v_{j}$ we can apply average pooling to obtain the global image feature $x$ as follows,

$$
x=\frac{1}{M} \sum_{j=1}^{M} v_{j}
$$

After the spatial pyramid pooling we have obtained a training dataset $X \in R^{F \times N}$ where $N$ is the number of training images and $F$ is the length of spatial pyramid pooled feature vector. Also we are given the image label matrix $Y \in\{0,1\}^{L \times N}$ where $L$ is the number of image categories. Then we can train a classifier using $X$ and $Y$ as follows,

$$
\arg \min _{W} \ell(W, X, Y)+\lambda_{3} \phi(W)
$$


where $W \in R^{F \times L}$ is the parameter of the classifier, $\ell(W, X, Y)$ is the loss function and $\phi(W)$ is the regularization term.

For simplicity we use the ridge regression loss function and the $\ell_{2}$ norm regularization term as follows,

$$
\arg \min _{W} J_{3}=\left\|Y-W^{T} X\right\|_{\ell_{2}}^{2}+\lambda_{3}\|W\|_{\ell_{2}}^{2}
$$

The solution to the ridge regression problem is in closed-form shown as follows,

$$
W=\left(X X^{T}+\lambda_{3} I\right)^{-1} X Y^{T}
$$

In this supervised learning visual phrase framework, the parameters to be optimized include the classifier weights matrix $W$, the visual phrase layer codebook $U$ and the visual primitive layer codebook $B$. Since the classifier weights matrix $W$ is in closed-form, we do not need the gradient descent based method to optimize it. Therefore we will focus on optimizing the other two variables $U$ and $B$ in the following discussions.

In order to update the codebook $U$ for the visual phrases, we need to calculate the derivative $\frac{\partial J_{3}}{\partial U}$ as follows,

$$
\frac{\partial J_{3}}{\partial U}=\sum_{k} \frac{\partial J_{3}}{\partial x_{k}} \times\left(\sum_{j} \frac{\partial x_{k}}{\partial v_{j}} \times \frac{\partial v_{j}}{\partial U}\right)
$$

where $\frac{\partial J_{3}}{\partial x_{k}}$ can be easily obtained from the ridge regression loss function in Eq. 2.20 and $\frac{\partial x_{k}}{\partial v_{j}}$ can also be easily obtained since it is only the spatial pyramid pooling from a set of local features $v_{j}$ to global features $x_{k}$. Therefore we only need to calculate $\frac{\partial v_{j}}{\partial U}$ to obtain $\frac{\partial J_{3}}{\partial U}$. Fortunately $\frac{\partial v_{j}}{\partial U}$ can be calculated using the implicit differentiation method as discussed in Sec. 2.3.1.6. 
In order to update the codebook $B$ for the visual primitives, we need to compute the derivative $\frac{\partial J_{3}}{\partial B}$ as follows,

$$
\frac{\partial J_{3}}{\partial B}=\sum_{k} \frac{\partial J_{3}}{\partial x_{k}} \times \sum_{j} \frac{\partial x_{k}}{\partial v_{j}} \times \frac{\partial v_{j}}{\partial z_{j}} \times \sum_{i} \frac{\partial z_{j}}{\partial c_{i}} \times \frac{\partial c_{i}}{\partial B}
$$

where the only unknown derivative so far is $\frac{\partial v_{j}}{\partial z_{j}}$. Fortunately we can also apply the implicit differentiation method in Eq. 2.4 to obtain the derivative $\frac{\partial v_{j}}{\partial z_{j}}$.

First we calculate the gradient with respect to $v_{j}$ at its minimum $\hat{v}_{j}$ :

$$
\left.2\left(U^{T} U v_{j}-U^{T} z_{j}\right)\right|_{v_{j}=\hat{v}_{j}}=-\lambda_{2} \times\left.\operatorname{sign}\left(v_{j}\right)\right|_{v_{j}=\hat{v}_{j}}
$$

Then we calculate the gradient with respect to $z_{j}$ on both sides of Eq. 2.24 as follows,

$$
\frac{\partial\left\{2\left(U^{T} U v_{j}-U^{T} z_{j}\right)\right\}}{\partial z_{j}}=\frac{\partial\left\{-\lambda_{2} \times \operatorname{sign}\left(v_{j}\right)\right\}}{\partial z_{j}}
$$

It is worth noting that the right-hand side of Eq. 2.25 is not well-defined at zero due to the non-continuous property of $\operatorname{sign}\left(v_{j}\right)$, therefore we choose the non-zero coefficients from $\hat{v}_{j}$ to form $\tilde{v}_{j}$ and select the corresponding codebook bases $\tilde{U}$ by $\tilde{v}_{j}$, and get the following result:

$$
\frac{\partial\left\{2\left(\tilde{U}^{T} \tilde{U} \tilde{v}_{j}-\tilde{U}^{T} z_{j}\right)\right\}}{\partial z_{j}}=0
$$

Since the visual phrase layer codebook $U$ is fixed while we try to update the visual primitive layer codebook $B$, we can expand Eq. 2.26 as follows,

$$
\frac{\partial \tilde{v}_{j}}{\partial z_{j}} \tilde{U}^{T} \tilde{U}-\tilde{U}=0
$$

which leads to the final result of $\frac{\partial v_{j}}{\partial z_{j}}$ :

$$
\frac{\partial \tilde{v}_{j}}{\partial z_{j}}=\tilde{U}\left(\tilde{U}^{T} \tilde{U}\right)^{-1}
$$




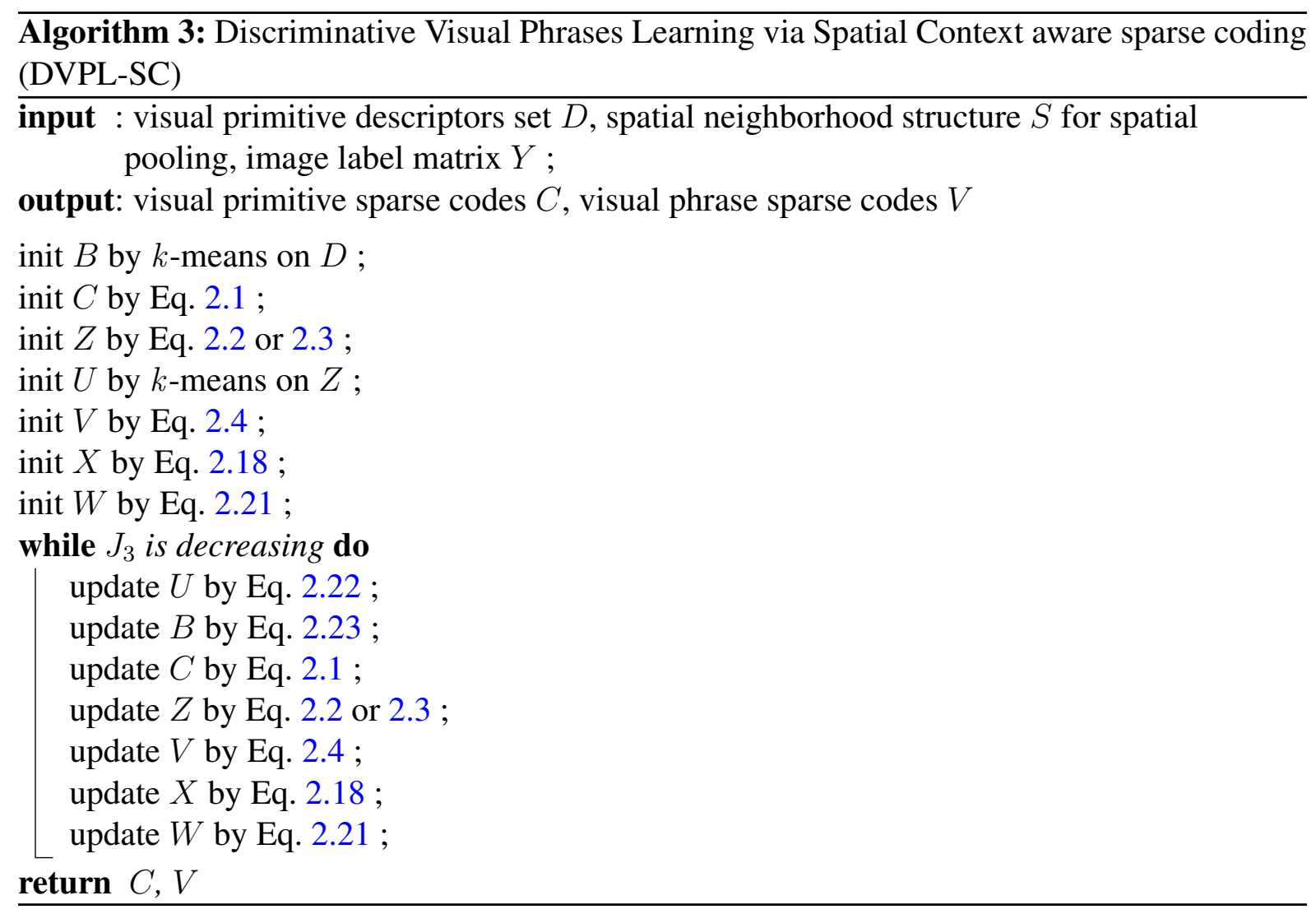

After that we can update the codebook $B$ of the visual primitives using the derivative $\frac{\partial J_{3}}{\partial B}$.

The discriminative learning process is shown in Alg. 3, where for simplicity we only show the discriminative visual phrase learning algorithm on single type of visual primitive feature. The extension to multiple visual primitive features is straightforward according to Alg. 2.

\subsection{Experiments}

\subsubsection{Texture Pattern Discovery}

In the first experiment, to illustrate the effectiveness of encoding spatial context information for discovering visual phrases, we evaluate the proposed VPL-SC algorithm on an LV bag image shown in Fig. 2.3. From the image we first extract in total 2985 SIFT points [1] as the visual primitives, upon which we compute the visual primitive sparse codes by Eq. 2.1 and the visual phrase sparse codes by the proposed VPL-SC algorithm. In the VPL-SC experiment, we 
construct the visual phrase descriptor using average pooling method of 8 nearest points around each SIFT point.

After learning the sparse codes, we perform $k$-means algorithm to cluster all the visual primitive/visual phrases into 4 image patterns. The results are shown in Table 2.1, where we use different colors shown in Fig. 2.3 to plot the SIFT points located at different image patterns.

From the results in Table 2.1, we can see that on the one hand, visual primitive sparse codes can hardly distinguish the SIFT points stemming from different visual patterns in the LV bag image. As shown in column (a), SIFT points that represent the same visual patterns may be separated into different clusters (e.g., the $1^{\text {st }}$ row and the $3^{\text {rd }}$ row), while a certain cluster may contain SIFT points that belong to different visual patterns (e.g., the $3^{\text {rd }}$ row). On the other hand, using visual phrase sparse codes we can discover exactly the 4 visual patterns in the LV bag image, as shown in column (b). This experiment justifies that our proposed VPL-SC algorithm can utilize the spatial context information to discover higher-level visual patterns in the image.

\subsubsection{Scene Clustering}

In the second experiment, to demonstrate the effectiveness of multi-feature fusion in our algorithm, we perform image scene clustering on the MSRC-V2 dataset [2]. We select a collection of 150 images from 5 scene categories: sheep, cow, aeroplane, boat, bicycle. Each image contains several region segmentations of the following 9 ones: grass, cow, sheep, sky, aeroplane, water, bicycle, road, boat. Sample images are shown in Fig. 2.4.

The ground-truth labeling of each region segmentation is provided by [59]. As shown in Fig. 2.5, multiple features have to be fused to distinguish different region segmentations, e.g., while color feature can distinguish sheep and cow, it cannot distinguish aeroplane, boat, or bike. Therefore following the work in [60] we describe each region segmentation with three features: Color Histogram (CH), Texton Histogram (TH) [60], and pyramid of HOG (pHOG) [61]. In the experiment, we consider region segmentations as visual primitives and the whole images as visual phrases in our algorithms. We use region segmentations in the same image as spatial 

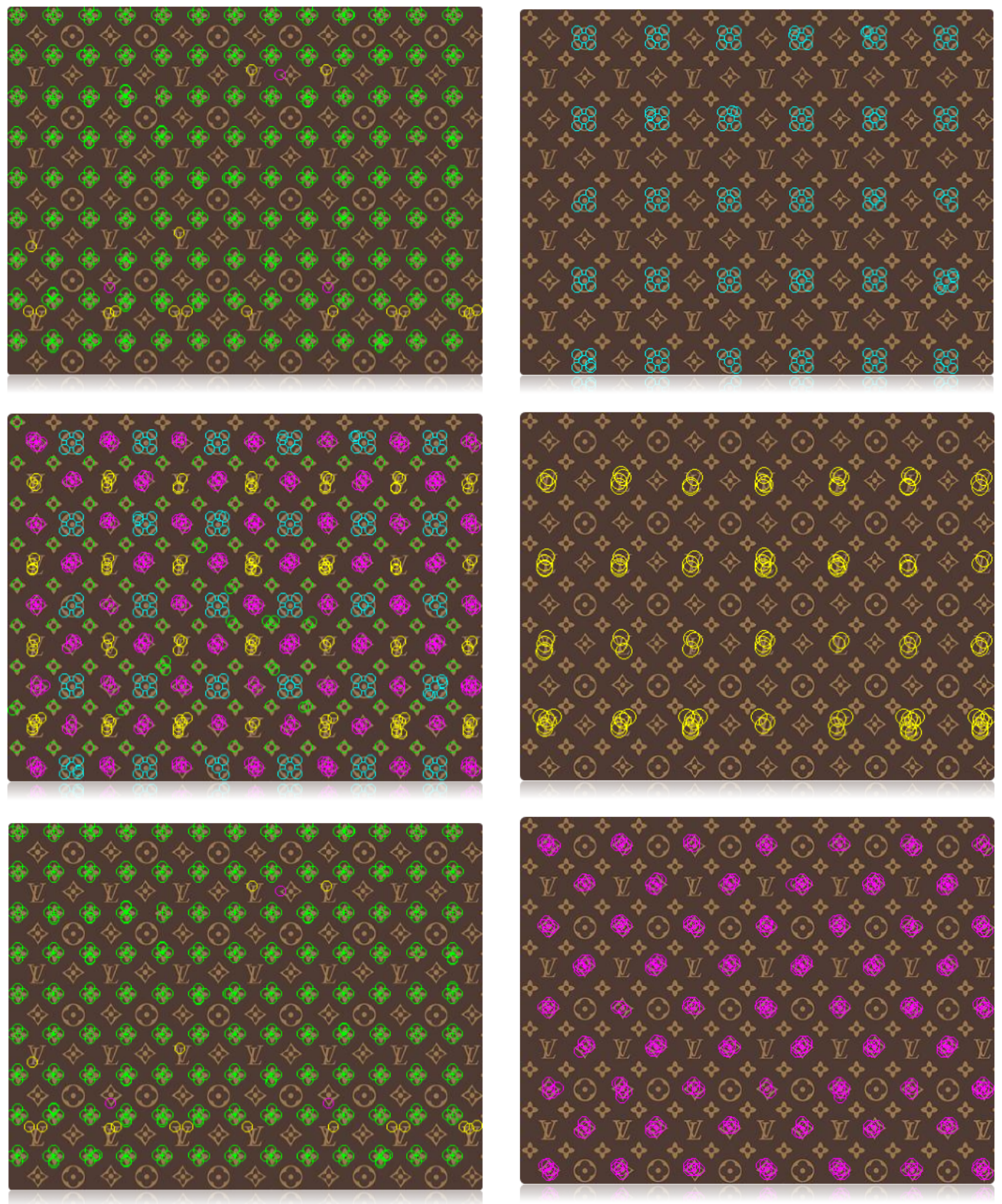

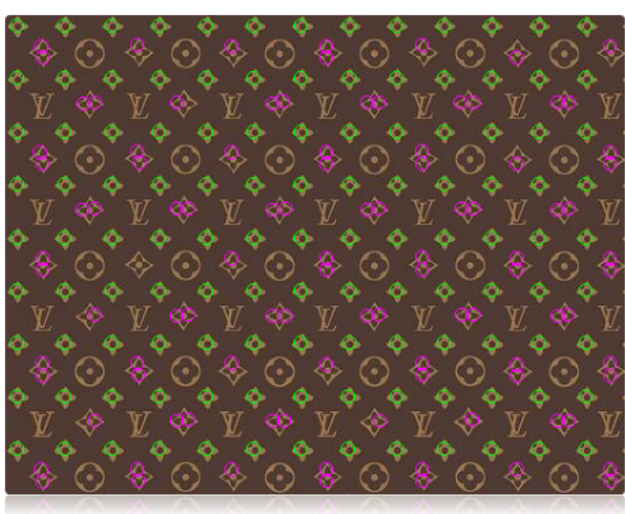

(a)

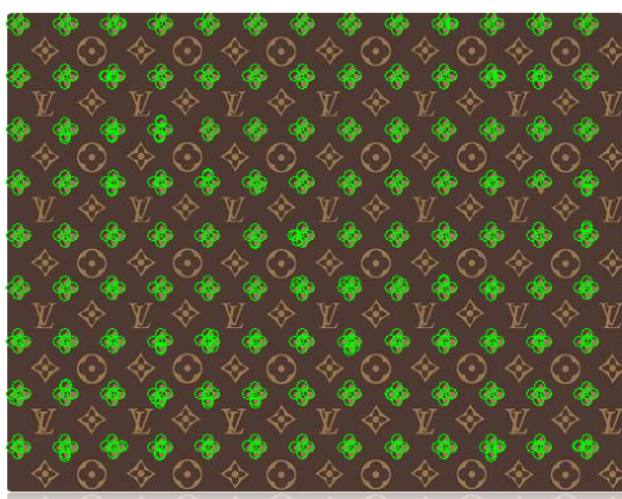

(b)

TABLE 2.1. Clustering results on the LV bag image by (a) sparse coding algorithm in Eq. 2.1; (b) our proposed VPL-SC method. 

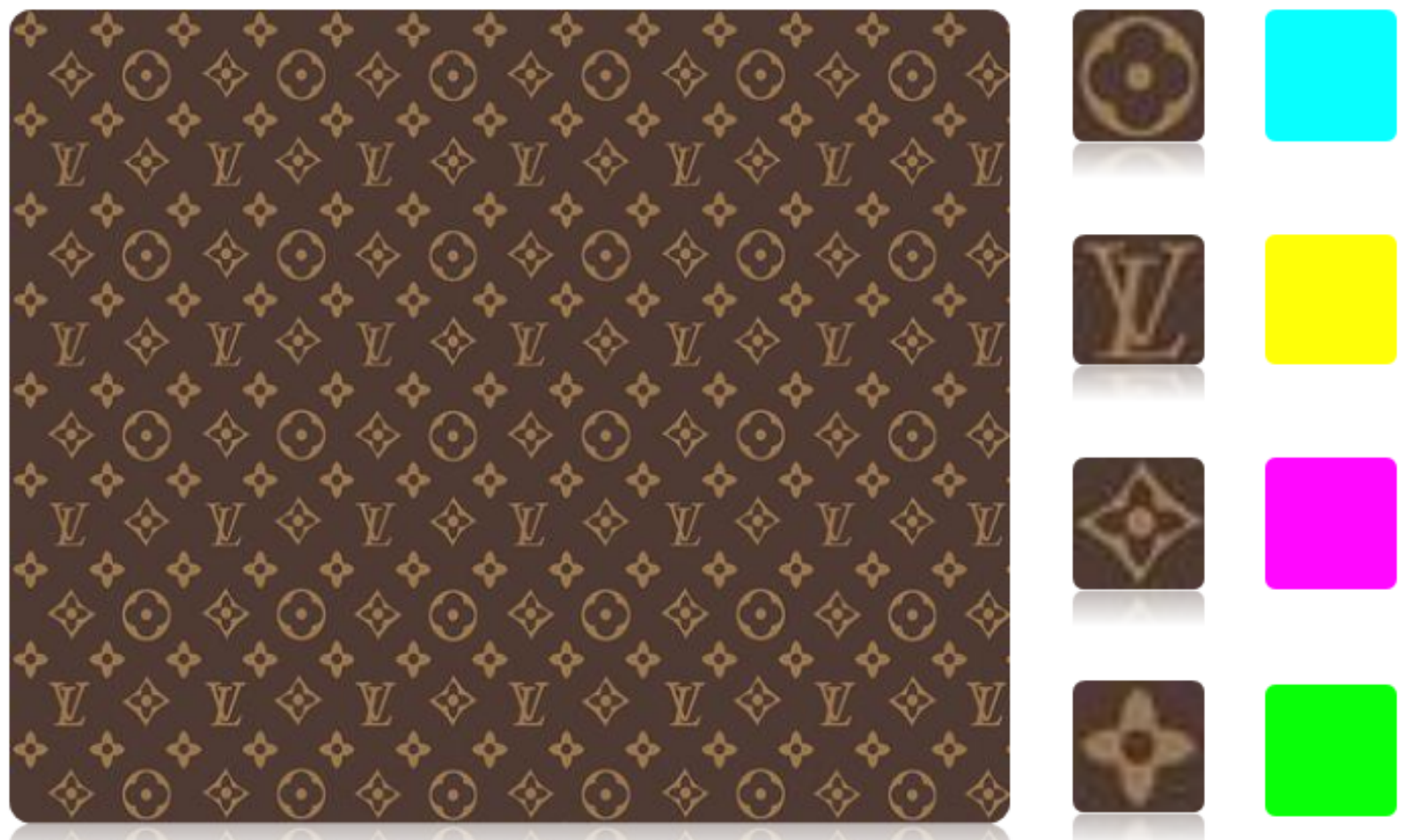

FIGURE 2.3. Illustration of an LV bag image. In the left we show the original image; In the middle we show the visual patterns contained in the original image; In the right we show the colors used for the visual primitives (e.g., SIFT points detected by [1]) located at different visual patterns.

neighbors for max pooling. After learning the visual phrases sparse codes, we perform $k$-means $(k=5)$ algorithm and evaluate the clustering performance by Hungarian matching algorithm.

\subsubsection{Comparing Multi-feature Fusion Results}

Table 2.2 shows the final clustering accuracy results, in which all the numbers are in \%. From the table we can see that, our proposed VPL-SC algorithm achieves slightly better performance $70 \%$ on the concatenated feature $\mathrm{TH}+\mathrm{CH}+\mathrm{pHOG}$ than $68 \%$ on the best individual feature $\mathrm{TH}$. However when the proposed multi-feature late fusion algorithm VPL-SC-MF is applied, it can significantly improve the performance over the best individual feature $\mathrm{TH}$ from $68 \%$ to $78.7 \%$. That is, the proposed multi-feature late fusion algorithm VPL-SC-MF can be more effective than the VPL-SC algorithm using single individual feature or using multi-feature early fusion to learn the visual phrase sparse codes. 
We also list the results from the related work [42], which proposed both spatial context aware and multi-feature fused regularized k-means method to discover mid-level visual phrases. From the results we can see that, our proposed VPL-SC algorithm successfully outperforms the kmeans algorithm used in [42] on all the individual features $\mathrm{TH}, \mathrm{CH}$ and $\mathrm{pHOG}$ and the concatenated feature $\mathrm{TH}+\mathrm{CH}+\mathrm{pHOG}$. Moreover our proposed algorithm VPL-SC-MF also significantly outperforms the multi-feature fusion method in [42] from $70.14 \%$ to $78.7 \%$.

\subsubsection{Comparing Visual Phrase Learning Results}

To evaluate the performance of using sparse coding method for the visual phrases, we also list the comparison results in the first and second columns in Table 2.2, where in the first column we use sparse coding method for the visual phrases and in the second column we only use the concatenation of raw features, i.e., without sparse coding for the visual primitives. As can be seen from the table, our proposed method that uses sparse coding for visual phrases outperforms the algorithm that uses only the concatenation of raw features, as used in [42]. For example, our method with visual phrase sparse coding improves the performance from $44.79 \%$ to $59.7 \%$ on $\mathrm{CH}$ feature, $55.69 \%$ to $68.0 \%$ on $\mathrm{TH}$ feature, $52.37 \%$ to $60.0 \%$ on pHOG feature, and $61.61 \%$ to $70.0 \%$ on $\mathrm{CH}+\mathrm{TH}+\mathrm{pHOG}$ feature. The experiment results have shown the advantages of our visual phrase learning method with sparse coding over the directly feature concatenation method in [42].

We also compare the performance with $\ell_{2,1}$-regularized term used in Eq. 2.4. As can be seen from the results, our method with $\ell_{1}$ regularized term outperformed $\ell_{2,1}$ regularized term, which indicated that $\ell_{2,1}$ term might be suited for feature selection [62], but not necessary an optimal choice to produce sparse codes.

We also list the results using VGG16 neural network [52] in Table 2.2. In the experiments, we use the fc7 layer of the CNN network to extract features. From the results we can see that our proposed method also outperforms [42] and [62] on high-level semantic convolution neural network features, which justifies the effectiveness of our proposed method. 


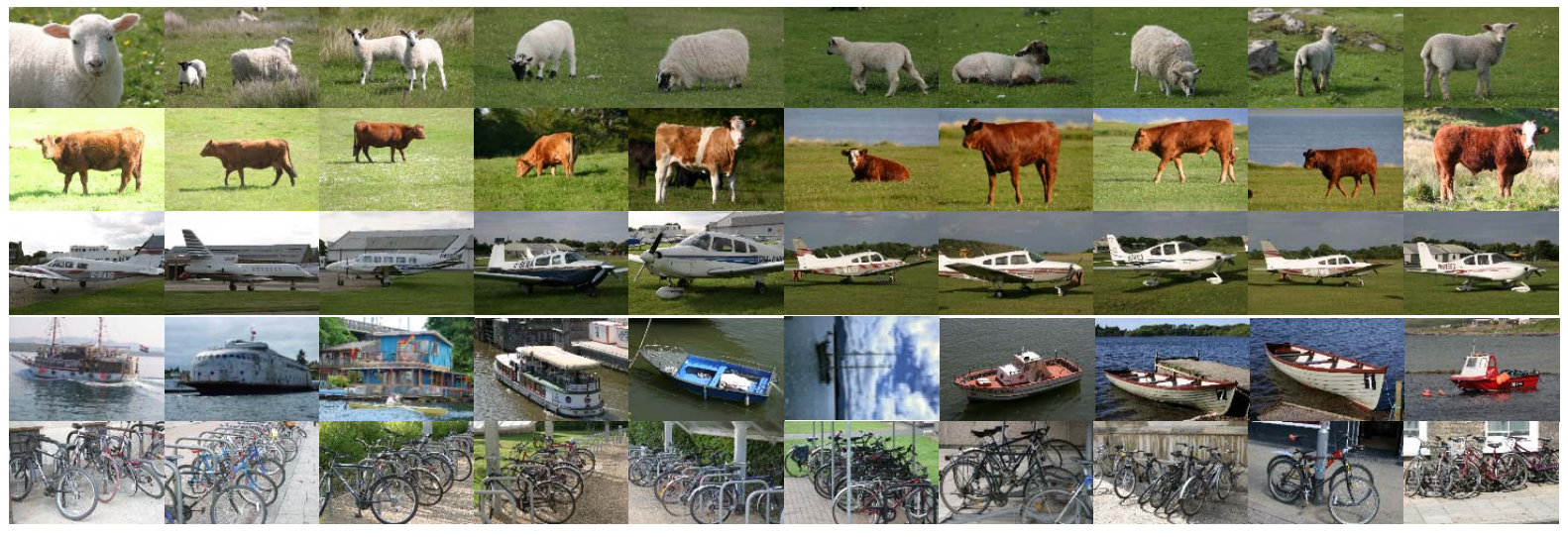

FIGURE 2.4. Sample images from MSRC-V2 dataset [2].

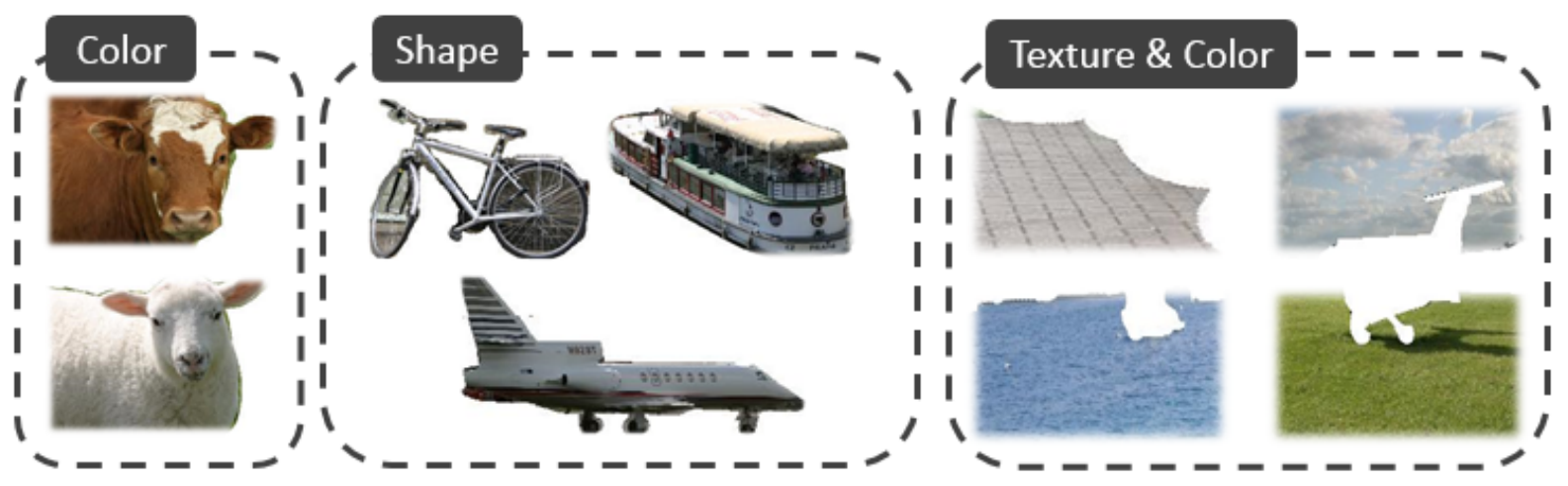

FIGURE 2.5. Illustration of different types of features used to distinguish different region segmentations.

\begin{tabular}{l|c|c|c|}
\hline Feature & VPL-SC & Context-Aware Discovery [42] & $\ell_{2,1}$-regularized [62] \\
\hline CH & 59.7 & 44.79 & 55.1 \\
TH & 68.0 & 55.69 & 61.8 \\
pHOG & 60.0 & 52.37 & 58.2 \\
TH+CH+pHOG & 70.0 & 61.61 & 64.5 \\
\hline Multi-feature Fusion & 78.7 & 70.14 & 72.2 \\
\hline CNN & $\mathbf{8 2 . 4}$ & 75.8 & 79.8 \\
\hline
\end{tabular}

TABLE 2.2. Clustering results on the MSRC-V2 dataset. 


\subsubsection{Scene Categorization on Scene-15 Dataset}

In the third experiment, to illustrate the effectiveness of our proposed discriminative visual phrase learning algorithm, we perform scene categorization on the Scene-15 dataset.

The Scene-15 dataset is gradually collected by $[22,63]$. It contains totally 4485 images from 15 categories of outdoor and indoor scenes, such as bedroom, living room, suburb, industrial, kitchen, coast, forest, highway, inside city, mountain, open country, street, tall building, office, and store. For each category, there are about 216 to 410 images of size about $300 \times 250$ pixels.

In the experiments we use 10 random split of the dataset and for each split we use 100 images from each category for training and the rest for testing. For the visual primitive layer, we extract dense SIFT [23] and dense edge-SIFT [47] as local descriptor sets on $16 \times 16$ pixel patches computed over a grid with spacing of 8 pixels. A codebook of size 1024 is trained upon the visual primitive layer. For the visual phrase layer, we apply $k$-NN at 4 scales, i.e., $k=4,8,12,16$, around each visual primitive descriptor for max pooling to construct the visual phrase layer descriptors. The codebook size of the visual phrase layer is also 1024. For the classifier layer, we use max pooling upon the spatial pyramid of $4 \times 4,2 \times 2,1 \times 1$ subregions to obtain the global image features for training, as used in $[22,23]$.

We run our proposed algorithm VPL-SC on the dense SIFT features, VPL-SC-MF and DVPLSC-MF on both dense SIFT and dense edge-SIFT features to learn visual phrase sparse codes respectively. After that, we further apply max pooling and spatial pyramid matching method upon the visual primitive sparse codes of different $k$-NN scales $(k=4,8,12,16)$ and the visual primitive sparse codes $(k=0)$ to form global image representations. Since different sparse codes have different discriminative power, we use multiple kernel learning algorithm with RBF kernels to train the final classifier.

Table 2.3 shows the final accuracy results. From the table we can see that our proposed VPL-SC algorithm outperforms previous sparse coding work [23] on the visual primitive layer by about $3 \%$, which justifies the discriminative power of our learnt visual phrases sparse codes. When compared with the Macrofeature work [27] that learned mid-level features on multiple visual 
primitive descriptors of local spatial neighborhood to encode the spatial context information, our proposed algorithm DVPL-SC-MF also shows superior recognition performance by about $1.3 \%$. Thanks to the proposed discriminative learning of visual phrase layer sparse codes and also the multi-feature fusion, our proposed DVPL-SC-MF algorithm achieves superior accuracy by $3.5 \%$, compared with the max-margin dictionary learning method on the visual primitive layer in [34]. The experiments on the Scene-15 dataset justify the advantages of our proposed discriminative visual phrase learning method that combines both spatial pooling and multifeature fusion techniques.

\begin{tabular}{l|c}
\hline Algorithm & Accuracy (\%) \\
\hline Kernel SPM [22] & 81.40 \\
Kernel Codebook [64] & 76.67 \\
Localized soft assignment [65] & 82.70 \\
LCSR [65] & 82.7 \\
Object bank [66] & 80.9 \\
Geometric phrase pooling [47] & 85.13 \\
$\ell_{p}$-norm pooling [16] & 83.20 \\
Kernel Descriptors [67] & $\mathbf{8 6 . 7}$ \\
ScSPM [23] & 80.28 \\
Macrofeatures [27] & 84.9 \\
Max-margin dictionary [34] & 82.7 \\
\hline VPL-SC (Ours) & 83.2 \\
VPL-SC-MF (Ours) & 85.5 \\
DVPL-SC-MF (Ours) & $\mathbf{8 6 . 2}$ \\
\hline
\end{tabular}

TABLE 2.3. Accuracy results on the Scene-15 dataset.

\subsection{Conclusion and Future Work}

We propose to learn discriminative mid-level visual phrase features via spatial context aware multi-feature sparse coding, upon low-level visual primitive features. With the help of labeled image dataset, we optimize the two layer sparse codes, as well as the two layer codebooks via back-propagation. Since we have utilized the spatial context information, multi-feature 
evidences information, and also the image label information, more representative and discriminative sparse codes of visual phrases can be obtained. Experiments on image pattern discovery, image scene clustering, scene categorization justify the effectiveness of our algorithms.

There are still many potential extensions of this work. For example, our current method uses human defined low-level visual primitives to discover mid-level visual phrases, it will be interesting if we can discover the visual patterns from convolution neural networks to take advantage of deep learning features.

It would also be interesting that we jointly discover visual patterns and learn image classifiers by multi-task learning from convolution neural networks directly. 



\section{Chapter 3}

\section{Discovering Class-specific Spatial Layout Patterns for Scene Recognition}

\subsection{Introduction}

Different from texts and audios analysis, the rich spatial information in images has been proved to play a critical role in scene recognition and object detection $[51,53,54,68]$. Specifically for scene recognition, many previous works have demonstrated that the discriminative power is limited without considering encoding spatial information for local visual primitive features [22, $24,69-73]$.

This is due to the fact that scene images are usually spatial compositions of foreground objects and background contexts with clear spatial layouts. Take the images in Fig. 3.1 for example, the "street" scene category often consists of "building" and "road" components, and the "coast" category is often composed by "sky", "coast", and "sea" components with clear spatial layouts. However, it remains a challenging problem to leverage the spatial layout information in scene recognition applications to boost the recognition performance.

In recent years, deep learning algorithms have been very popular for scene/object recognition due to the state-of-the-art performances $[51,53,54]$. For example, the AlexNet model [51] 

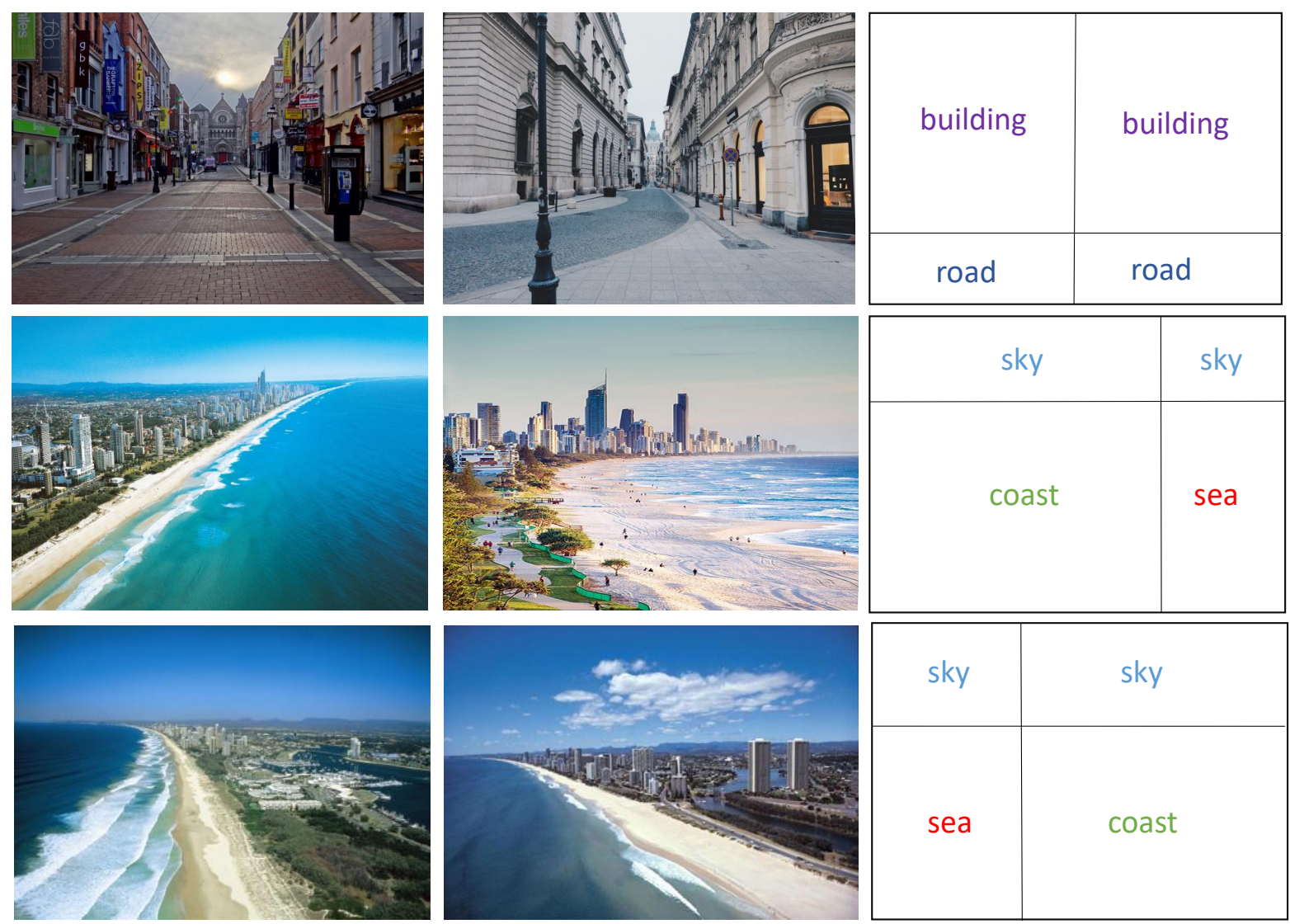

FIGURE 3.1. Illustration of the spatial layouts for street and coast scene categories. Comparing the $1^{\text {st }}$ row to the $2^{\text {nd }}$ and $3^{\text {rd }}$ rows, we can see that street and coast categories have different spatial layouts due to large inter-class variations. From the $2^{\text {nd }}$ and $3^{\text {rd }}$ rows, we can see that within even the same category, images can also exhibit different spatial layouts due to large intra-class variations.

trained on 1.2 million images of 1000 classes achieved the $1^{\text {st }}$ place with top-5 test error rate $15.3 \%$ compared to the $26.2 \%$ achieved by the $2^{\text {nd }}$ best method on the ILSVRC 2012 challenge. The ResNet work [54] achieved 3.57\% error on the ImageNet test set and won the $1^{\text {st }}$ place on the ILSVRC 2015 classification task. The Places-CNN work in [53] has been proposed to learn convolution neural network features for the large scale scene recognition task instead of the object recognition task on the ILSVRC challenge. The SPPNet work in [24] also proposed to use the spatial pyramid pooling for the fully connected layers to utilize the spatial layout information and boosted the accuracy of a variety of CNN networks in spite of their different architecture designs. 
In spite of the great successes of previous works, there still exist many limitations. The importance of class-specific global spatial layout of scene image has not been explicitly explored in many methods [51, 53]. First, the class-generic pre-defined spatial layout is applied in many previous spatial pyramid pooling methods [22, 24, 74], but is not necessarily an optimal choice for classification. This is due to the large inter-class variations among different scene categories. As shown in Fig. 3.1, the "street" scene images have different spatial layout compared to the "coast" scene images and therefore the class-generic spatial layout can not work well for both scene categories. In such a case, class-specific spatial layout should be considered. Second, due to the large intra-class variations, scene images from even the same category could exhibit different spatial layouts. As a result, a unique spatial layout may not be optimal to capture all the variations. From Fig. 3.1, the images in the $2^{\text {nd }}$ and $3^{\text {rd }}$ row are both from the "coast" category but they clearly have different spatial layouts.

One of the most intuitive ways to leverage the spatial layout information is to partition the image space into pre-defined grid cells and then compute the corresponding visual features for each grid cell and finally concatenate them all to form a global image representation. For example, the spatial pyramid matching [22] method using hand-crafted features such as SIFT and HOG has illustrated the effectiveness of encoding the spatial pyramid information. It significantly improved the classification performance of the previous bag-of-visual-word method [25]. The SPP-Net work in [24] also proposed to use the spatial pyramid pooling for the fully connected layers to utilize the spatial layout information and boosted the accuracy of a variety of $\mathrm{CN}$ $\mathrm{N}$ networks in spite of their different architecture designs. The work in [69] also proposed a boosting method to select discriminative visual features under different spatial layouts for scene recognition and achieved superior performance compared to previous methods without explicitly using spatial layout information.

This chapter contributes to addressing the above issues. We propose to discover the classspecific global spatial layouts of scene images to boost the recognition performance. We first generate multiple random spatial layouts and then propose to jointly learn the class-specific spatial layouts and the image classifier by solving an $\ell_{1}$-regularized max-margin optimization 
problem. The objective function can be optimized and converge to a local optima by our proposed alternating method. The introduced $\ell_{1}$ regularized term induces sparsity of the discovered class-specific spatial layouts. As a result we are able to discover a sparse combination of class-specific spatial layouts and achieve superior performance compared to the method without explicitly considering the spatial layout information. Also thanks to the use of deep learning features [54] instead of traditional hand-crafted features, our method achieves significant improvement over previous methods. Experiments on Scene-15, Landuse-21 and MIT indoor-67 datasets validate the advantages of our proposed algorithms.

The remainder of this chapter is organized as follows. In Sec. 3.2 we provide our feature extraction approaches, and then propose our discovery of class-specific spatial layout algorithms. In Sec. 3.3 we conduct experiments on the Scene-15 dataset, Landuse-21 dataset and MIT indoor-67 dataset. In Sec. 3.4 we have a conclusion.

\subsection{Proposed Method}

Let us declare the notations first. We use upper case letter in bold font to denote a matrix, e.g., $\mathrm{X}$. Lower case letter in bold font is used to represent a vector, e.g., $\mathbf{x}$. We use lower case letter in normal font for a scalar, e.g., $y$. We also use the notations $\left\||\cdot \||_{2}\right.$ as $\ell_{2}$-norm and $\||\cdot \||_{1}$ as $\ell_{1}$-norm in the following sections.

\subsubsection{Feature Extraction}

In this section, we will also introduce the feature extraction approach using ConvNet for our proposed methods. As shown in Fig. 3.2, first we obtain a set of 2D feature maps by forwarding the images through the convolution and pooling layers of the ConvNet, then we apply multiple random partitioning to the $2 \mathrm{D}$ feature maps. After that, for each feature map we can obtain a concatenated fully-connected feature vector, and finally we stack all the feature vectors to get the final matrix representation for each image. 
Formally, we define a spatial pyramid of $\ell$ levels and each level $i$ is randomly partitioned into non-overlapping $2^{i} \times 2^{i}$ sub-regions. We do the random partitions by $m$ times to obtain different grid cells to construct the spatial pyramids. As a result, we can obtain a set of sub-regions $R=\left\{R_{i j} \mid \forall i \in\{1, \cdots, n\}, \forall j \in\{1, \cdots, m\}\right\}$ where $n=\frac{1}{3}\left(4^{\ell+1}-1\right)$ is the number of sub-regions for each pyramid and $m$ is the number of random partitions. Then we input these sub-regions into the ConvNet [54] to get the corresponding feature vector $\mathbf{x}_{\mathbf{i j}} \in \mathbb{R}^{d}$ (column vector) for each sub-region $R_{i j}$.

After that, we stack the feature vectors for different random partitions and define the data matrix $\mathbf{X} \in \mathbb{R}^{n d \times m}$ for each image in Eq. 3.1, where each column vector $\mathbf{x}_{i} \in \mathbb{R}^{n d}$ is the feature representation for each random partition and each $\mathbf{x}_{i j}$ is the $i^{\text {th }}$ sub-region under the $j^{\text {th }}$ random partition.

$$
\mathbf{X}=\left[\mathbf{x}_{1}, \cdots, \mathbf{x}_{m}\right]=\left[\begin{array}{ccc}
\mathbf{x}_{11} & \cdots & \mathbf{x}_{1 m} \\
\vdots & \ddots & \vdots \\
\mathbf{x}_{n 1} & \cdots & \mathbf{x}_{n m}
\end{array}\right]
$$

Note that we explicitly encode the spatial layout information in the matrix representation for each image using multiple random partitions. The remaining problem is how to learn which spatial layouts are the optimal ones for each class-specific scene.

\subsubsection{Proposed Method}

In this section, we will first introduce the proposed method to discover class-specific spatial layouts for each class given the data matrix $\mathbf{X}$ extracted from Sec. 3.2.1 for each image.

For a particular scene category, due to the large intra-class variations, the optimal spatial layout may not be a unique one. We thus assume that the best spatial layout should be a mixture of spatial layouts, i.e., a linear combination of spatial layouts as follows,

$$
\mathbf{z}=\sum_{i=1}^{m} v_{i} \mathbf{x}_{\mathbf{i}}=\mathbf{X} \mathbf{v}
$$




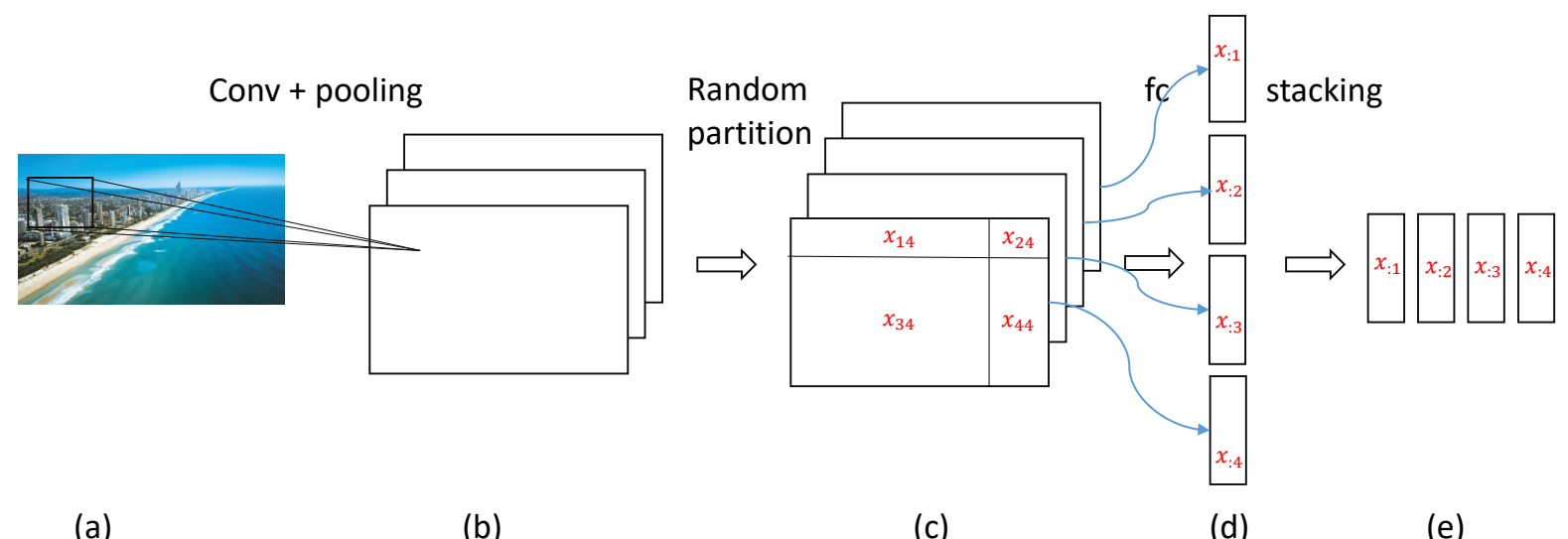

FIGURE 3.2. Illustration of the feature extraction pipeline for the proposed method. (a) shows the input image. (b) shows the 2D feature maps of the convolution and pooling layers of the convnets. (c) shows the multiple random partitions to form different spatial layouts. (d) shows the extracted features using the fc layer for different partitions. (e) shows the stacked data matrix for the input image. From the figure we can see that, we insert a random partitioning layer between the convolution/pooling layers and the fc layers of the convnets, and finally output a stacked data matrix as the data representation for each image.

where $\mathbf{v}=\left[v_{1}, v_{2}, \cdots, v_{m}\right]^{T} \in \mathbb{R}^{m}$ is the coefficient weight for the $m$ different random partitions and $\mathbf{z} \in \mathbb{R}^{n d}$ is the resulting feature representation for the image pyramid of in total $n$ sub-regions.

Once we have obtained $\mathbf{z}$, we are interested in training a linear SVM classifier as follows:

$$
f(\mathbf{X})=\mathbf{u}^{T} \mathbf{z}=\mathbf{u}^{T} \mathbf{X} \mathbf{v}
$$

where $\mathbf{u}=\left[u_{1}, u_{2}, \cdots, u_{n d}\right]^{T} \in \mathbb{R}^{n d}$ is the linear SVM weight vector of length $n d$. Note that we skip the bias terms to simplify the notations. It is also worth noting that the parameters number in the form of $f(\cdot)$ is $|\mathbf{u}|+|\mathbf{v}|$, compared to $|\mathbf{u}| *|\mathbf{v}|$ if we learn a linear SVM by concatenating all the columns of data matrix $\mathbf{X}$. The latter case is either prone to overfitting on the training dataset or infeasible due to the computational resource limitation, e.g., the lack of enough RAM or the need for much longer running time. 
We follow the work on support vector machines [75] and define the empirical loss of classifier $f(\cdot)$ as the sum of the square hinge losses over a collection of $T$ training images:

$$
\sum_{t=1}^{T} \max \left(0,1-y_{t} f\left(\mathbf{X}^{t}\right)\right)^{2}
$$

where $\mathbf{X}^{t}$ is the data matrix as described in Sec. 3.2.1 for the $t^{\text {th }}$ image and $y_{t} \in\{1,-1\}$ is the corresponding label. Note that we use the square hinge loss instead of the hinge loss following the work in [75] to simplify the computation.

Since focusing solely on the empirical loss may result in over-fitting the training set, we also add an $\ell_{2}$-norm regularization on the classifier weight $\mathbf{u}$ and an $\ell_{1}$-norm regularization on the partition coefficient $\mathbf{v}$, then the final objective function becomes as follows:

$$
\begin{array}{r}
\arg \min _{\mathbf{u}, \mathbf{v}} \frac{1}{2}\|\mathbf{u}\|^{2}+\lambda\|\mathbf{v}\|_{1}+C \sum_{t=1}^{T} \max \left(0,1-y_{t} f\left(\mathbf{X}^{t}\right)\right)^{2} \\
f(\mathbf{X})=\mathbf{u}^{T} \mathbf{X} \mathbf{v}
\end{array}
$$

where $\lambda$ is the parameter for the $\ell_{1}$-norm regularization $\|\mid v\|_{1}$ and $C$ is the parameter for the hinge loss on the $T$ training samples.

It is worth noting that we use the traditional $\ell_{2}$-norm regularization for the weight $\mathbf{u}$ to learn the image classifier, and also the $\ell_{1}$-norm regularization for the weight $\mathbf{v}$ to learn the combinations of random partitions. This is due to the consideration that the $\ell_{2}$-norm regularization on the weight $\mathbf{u}$ follows the max-margin framework [76], while the $\ell_{1}$-norm regularization on the coefficient vector $\mathbf{v}$ induces sparsity under certain conditions [77]. As a result, we can learn the class-specific weights $\mathbf{u}$ and $\mathbf{v}$ for different scene categories with different appearance features and different spatial layouts.

To optimize the loss function (3.3), we use an alternating method to iteratively optimize $\mathbf{u}$ and $\mathbf{v}$. When $\mathbf{v}$ is fixed, training $\mathbf{u}$ becomes an $\ell_{2}$-regularized $\ell_{2}$-loss $S V M$ problem as shown in 
Eq. 3.5.

$$
\begin{array}{r}
\arg \min _{\mathbf{u}} \frac{1}{2}\|\mathbf{u}\|^{2}+C \sum_{t=1}^{T} \max \left(0,1-y_{t} f\left(\mathbf{X}^{t}\right)\right)^{2} \\
f(\mathbf{X})=\mathbf{u}^{T}(\mathbf{X} \mathbf{v})
\end{array}
$$

Similarly once $\mathbf{u}$ is fixed, updating $\mathbf{v}$ also reduces to an $\ell_{1}$-regularized $\ell_{2}$-loss problem, as shown in Eq. 3.7.

$$
\begin{array}{r}
\arg \min _{\mathbf{v}} \lambda\|\mathbf{v}\|_{1}+C \sum_{i=t}^{T} \max \left(0,1-y_{t} f\left(\mathbf{X}^{t}\right)\right)^{2} \\
f(\mathbf{X})=\left(\mathbf{u}^{T} \mathbf{X}\right) \mathbf{v}
\end{array}
$$

Intuitively, optimizing $\mathbf{u}$ can be viewed as the conventional linear SVM classifier training process, given the feature vector $\mathbf{X v}$ for each image, while on the other hand, updating $\mathbf{v}$ can be viewed as learning the sparse random partition coefficients, given the feature vector $\mathbf{u}^{T} \mathbf{X}$ for each image. To discover class-specific spatial layouts for multi-class dataset, we apply onev.s.-rest training manner. Although there exist many multi-class SVM training strategies such as [78-80], we choose the simple one with one-v.s.-rest training mechanism such that we can separately learn the class-specific linear classifier weight $\mathbf{u}$ and the spatial layout weight $\mathbf{v}$ for each scene category. The Alg. 4 shows the complete alternating optimization method. For each iteration, the proposed objective function can reduce to one traditional SVM problem and one $\ell_{1}$-regularized quadratic programming $(\mathrm{QP})$ problem, therefore the complexity of the proposed alternating optimization method is the summation of the complexities of the traditional SVM problem and the $\ell_{1}$-regularized QP problem. The objective can guarantee to gradually converge to a local optima by the proposed alternating optimization method. In our experiments, we find the objective usually converges in less than 20 iterations. 


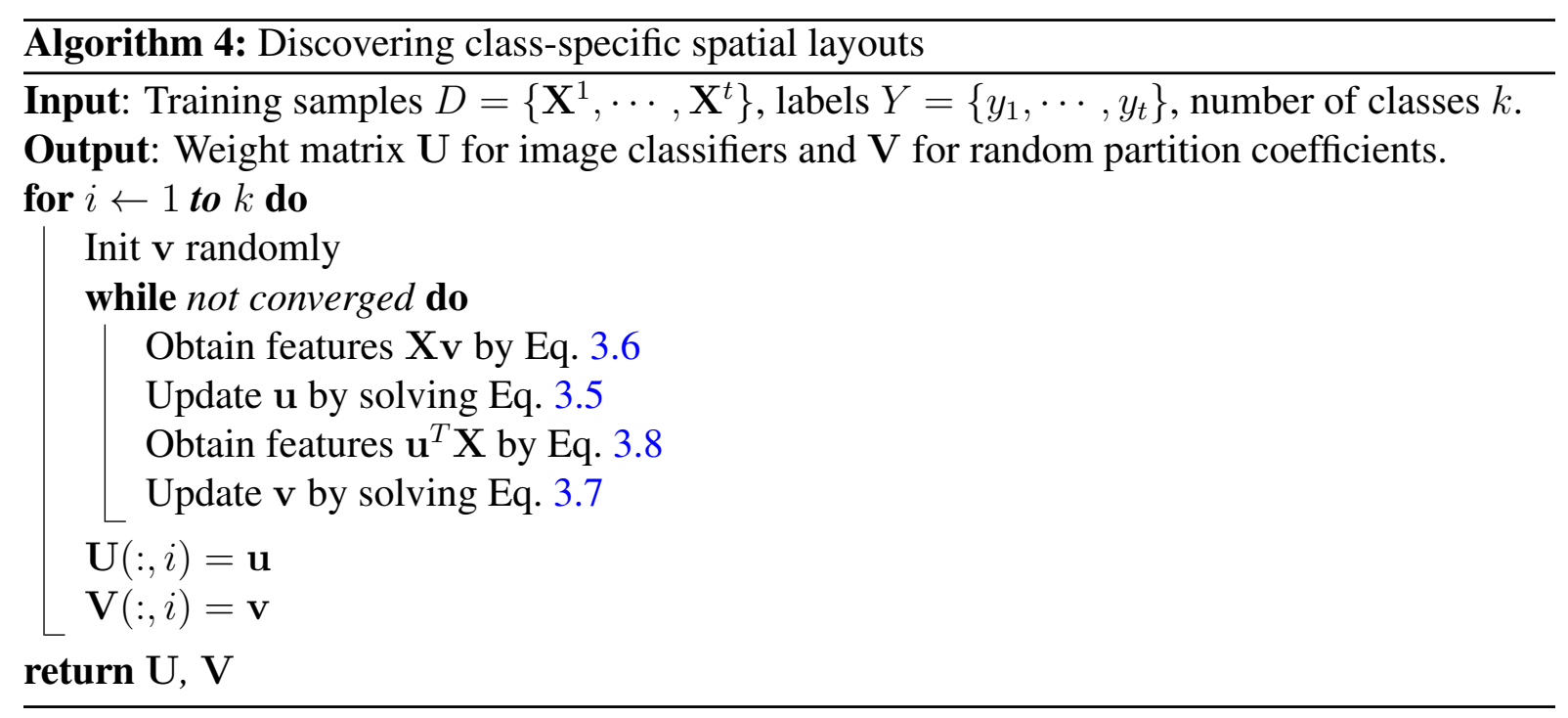

\subsection{Experiment}

In this section, we evaluate our proposed class-specific spatial layouts learning algorithm on Scene-15, Landuse-21 and MIT indoor-67 datasets for scene recognition. In the experiments, we use the Resnet-152 network [54] to extract features for each image. Each input of the network is first resized to $224 \times 224$ pixels and then forwarded through the network until the "pool5" layer and finally output as a 2048-dimension feature vector. In the experiments, we tune the parameters $\lambda$ and $C$ in Eq. 3.3 by grid search on the validation set. For the times of random partitions, we simply set it to 30 since there are already many overlaps among different spatial layouts. In the experiments we found the final performance does not change much when increasing the number of spatial layouts.

\subsubsection{Scene-15 Dataset}

The Scene-15 dataset [22,63] contains a variety of indoor and outdoor scenes. There are 216 to 410 images in each category with the resolution about $300 \times 250$. In the experiments, we use 3 -level spatial pyramid, i.e., the $1 \times 1,2 \times 2,4 \times 4$ structures, and we randomly partition the images by 30 times. To incorporate the spatial pyramid pooling [22], we also include the evenly partitioned spatial layout as one of the 30 random partitions. Following the same settings in [22], we use 100 images per class for training and the rest for testing. 


\begin{tabular}{l|c}
\hline Algorithm & Accuracy (\%) \\
\hline Linear SPM [23] & 65.32 \\
Kernel SPM [22] & 81.40 \\
Kernel Codebook [64] & 76.67 \\
Sparse Coding SPM [23] & 80.28 \\
Locality Linear Coding [32] & 79.24 \\
Geometric $\ell_{p}$-norm pooling [16] & 83.20 \\
Boosting + ORSP [69] & 83.9 \\
Boosting + BRSP [69] & 88.1 \\
Data-driven LBP [69] & 87.2 \\
\hline Convnets + SVM [53] & 84.2 \\
Resnet + SVM & 92.29 \\
Resnet + Weighted Layout (Ours) & $\mathbf{9 4 . 4 7}$ \\
\hline
\end{tabular}

TABLE 3.1. Accuracy results on the Scene-15 dataset.

Table 3.1 compares the results of our proposed method and other related methods. From the table we can see that, our proposed method explicitly utilizes the class-specific spatial layout information and achieves better performance than the direct competitor that uses the same features, i.e., the Resnet + SVM method. And thanks to the use of Resnet-152 features [54] and the class-specific spatial layouts, our method achieves the best performance among the methods using both traditional hand-crafted features and deep learning features. It is also worth noting that, our proposed method significantly outperforms the previous Boosting + ORSP/BRSP methods which encoded different spatial layout information into different patterns and then applied feature selection method to find the discriminative patterns for classification. We show the confusion matrix for the Scene-15 dataset in Fig. 3.3.

The random partition coefficients matrix $\mathrm{V}$ in absolute values for 15 classes is also shown in Fig. 3.4. We can see that, the discovered random partition coefficients are sparse vectors, e.g., class $1-9,11,14,15$, which justifies the use of $\ell_{1}$-norm regularization in Eq. 3.7. As for class $10,12,13$, although the coefficient vectors are not so sparse, they are very different from other classes, which can lead to better classification performance. We also find that the discovered spatial layouts are discriminative if we compare one to the other, i.e., using our proposed method we can select the class-specific spatial layouts which are discriminative for classification. It is also interesting to see that the $1^{\text {st }}$ row, i.e., the evenly partitioned spatial 


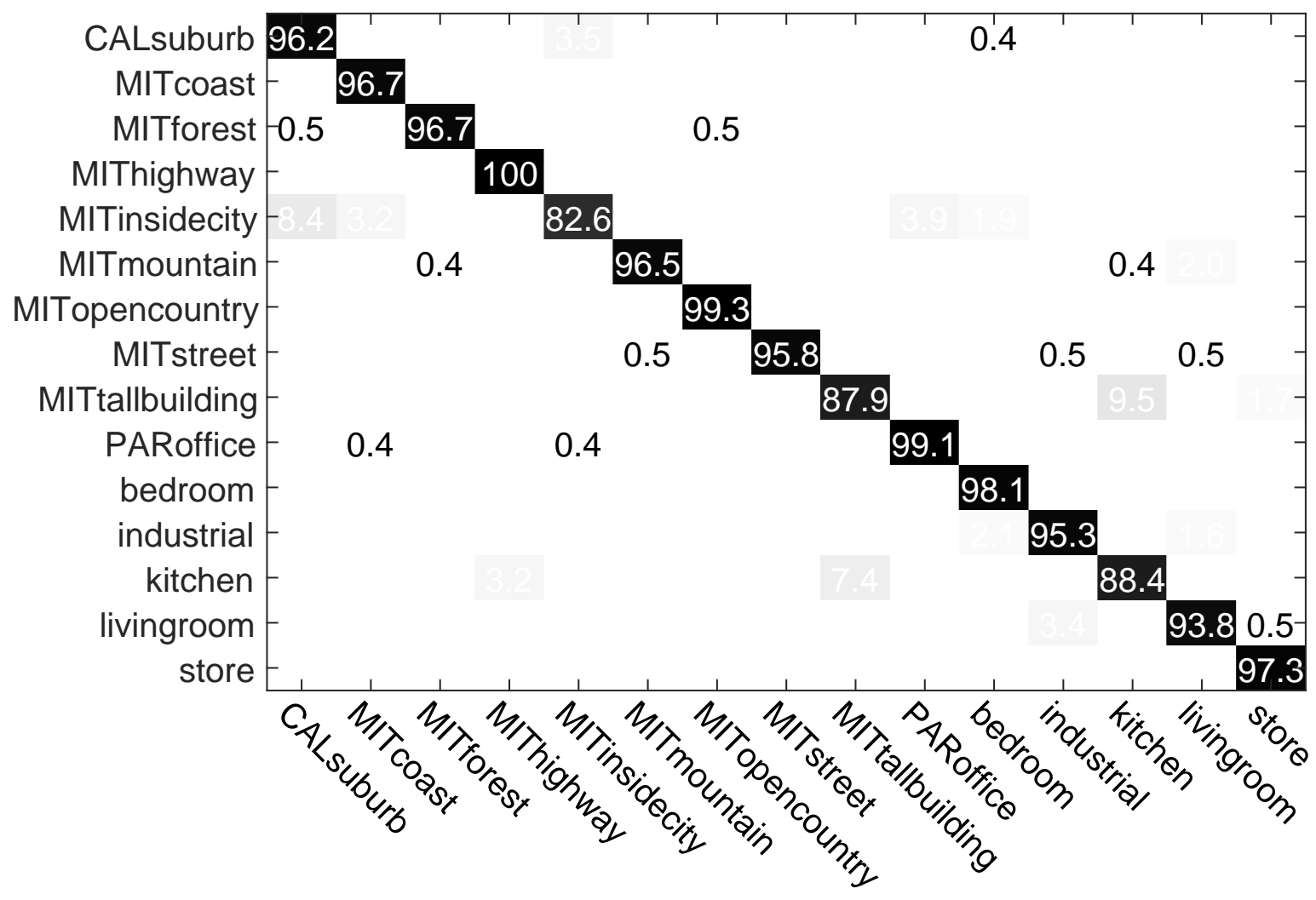

FIgURE 3.3. Confusion matrix for Scene-15 dataset.

layout, contributes more to the coefficients for each scene category, which suggests that the evenly partitioned spatial layout can be a good baseline and other spatial layouts can help further improve the final scene recognition performance. The accuracy results in Table 3.1 and the spatial layout weights in Fig. 3.4 justify our proposed method that jointly learns the image classifier weights and the class-specific spatial layout weights.

\subsubsection{Landuse-21 Dataset}

The Landuse-21 dataset [81] consists of 21 classes of aerial orthoimagery from the United States Geological Survey (USGS) National Map. In the dataset, there are 100 images for each class and the resolution is $256 \times 256$. As for training and testing split, we follow the settings in [81] and use 80 images for each class for training and the rest 20 for testing. The spatial layout settings are the same as used in the Scene- 15 experiments. 


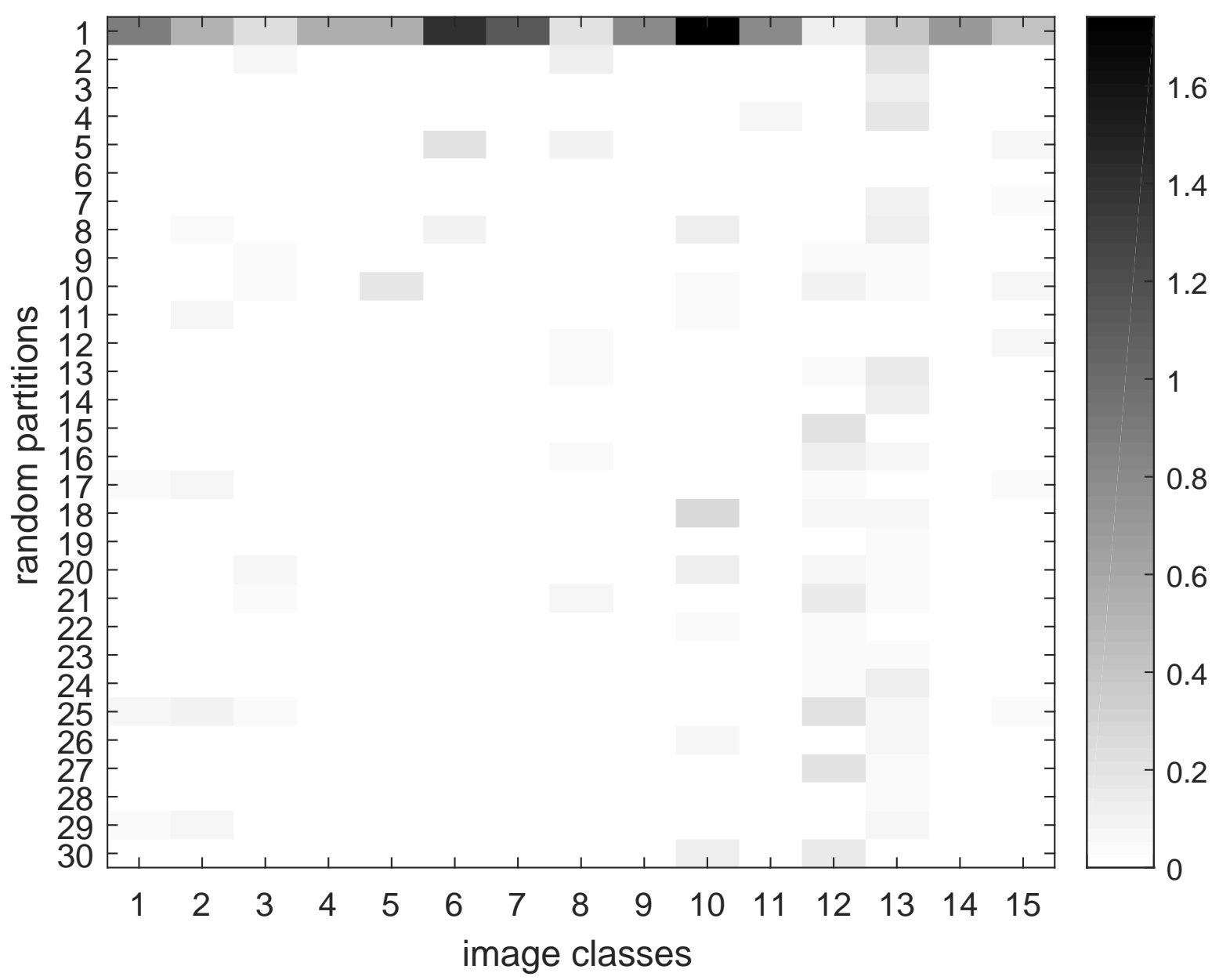

FIGURE 3.4. Random partition coefficients for Scene-15 dataset.

The results of our proposed method are shown in Table 3.2. The confusion matrix is also shown in Fig. 3.5. From the table we can see that, our proposed method significantly outperforms previous hand-crafted features and also we can improve the baseline Resnet + SVM method. The confusion matrix of Landuse-21 dataset is shown in Fig. 3.5.

\subsubsection{MIT Indoor-67 Dataset}

The MIT Indoor-67 dataset [82] consists of 67 classes of indoor scene images. In the dataset, there are different types of stores (e.g., bakery, grocery), residential rooms (e.g., nursery room, bedroom), public spaces (e.g., inside bus, library, prison cell), leisure places (e.g., buffet, fast food, bar, movie theater) and working places (e.g., office, operating room, tv studio). 


\begin{tabular}{l|c}
\hline Algorithm & Accuracy (\%) \\
\hline BoVW [69] & 71.9 \\
Kernel SPM [22] & 74.0 \\
SPCK [81] & 73.1 \\
SPCK+[81] & 76.1 \\
SPCK++ [81] & 79.24 \\
Boosting + ORSP [69] & 77.3 \\
Boosting + BRSP [69] & 75.5 \\
\hline Resnet + SVM & 98.10 \\
Resnet + Weighted Layout (Ours) & $\mathbf{9 8 . 5 7}$ \\
\hline
\end{tabular}

TABLE 3.2. Accuracy results on the Landuse-21 dataset.

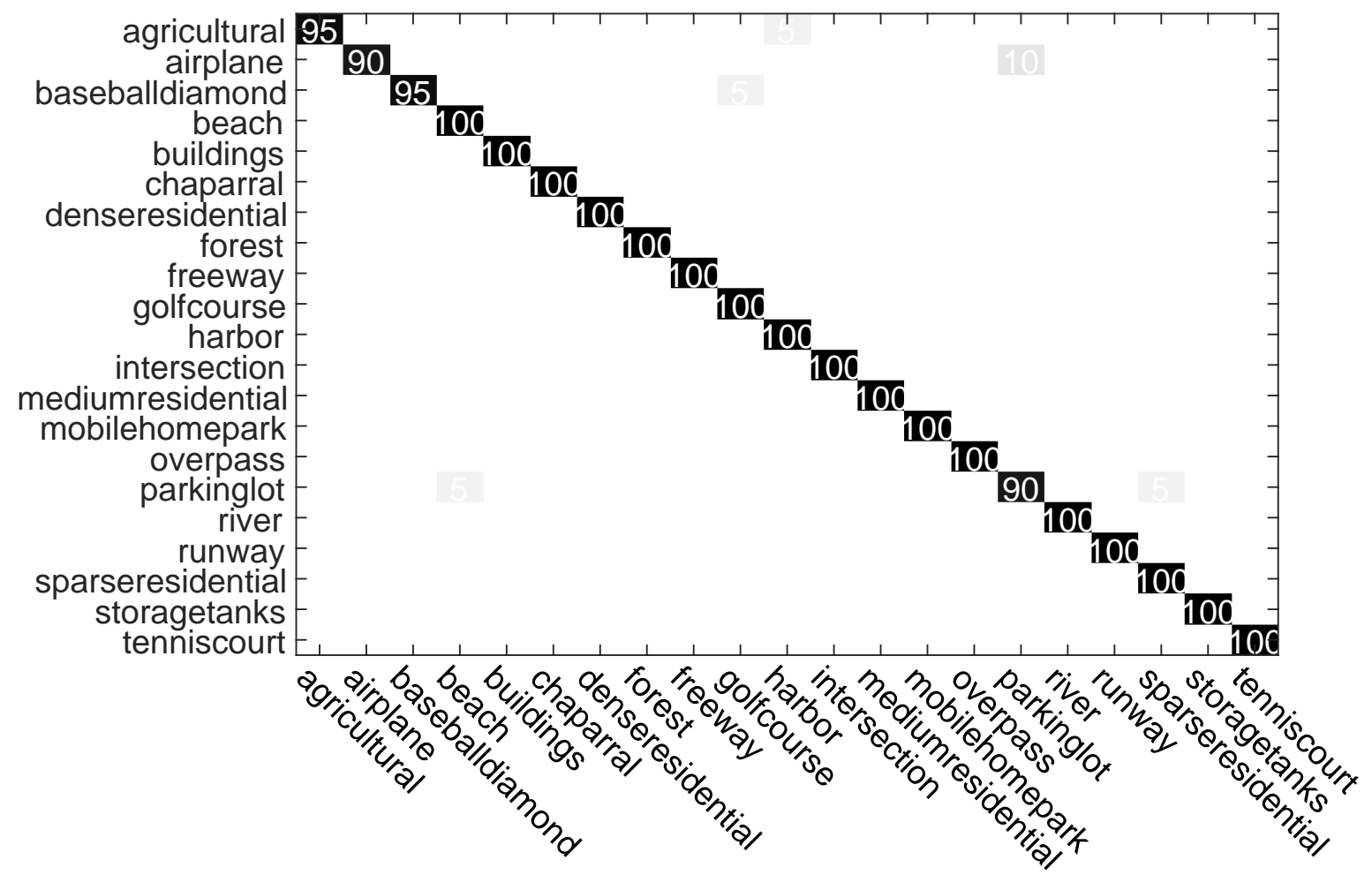

FIGURE 3.5. Confusion matrix for Landuse-21 dataset. 
Due to the large number of classes, and the large inter-class variation, it is a more challenging dataset compared to the Scene-15 and Landuse-21 datasets. In the experiments, we use the standard training and testing split from [82], i.e., 80 images per class for training and 20 images per class for testing. The spatial layout settings are also the same as used in the Scene-15 experiments.

\begin{tabular}{l|c}
\hline Algorithm & Accuracy (\%) \\
\hline D-Parts[83] & 51.4 \\
IFV [84] & 60.8 \\
MLrep [85] & 64.0 \\
\hline Convnets + SVM [53] & 58.4 \\
Resnet + SVM & 78.88 \\
Resnet + Weighted Layout (Ours) & $\mathbf{8 0 . 9 7}$ \\
\hline
\end{tabular}

TABLE 3.3. Accuracy results on the MIT Indoor-67 dataset.

The results of our proposed method are shown in Table 3.3. As can be seen from the table, our proposed method achieves the best performance among the methods using both hand-crafted features and deep convolution features. It is worth noting that, our method can still improve the Resnet + SVM method by about $2 \%$ on such a big dataset with 67 classes of indoor scenes, which further justifies the advantages of our proposed method.

\subsection{Conclusion and Future Work}

Finding discriminative spatial layouts is critical to scene recognition. To discover class-specific global spatial layouts of image content for scene recognition, we first generate random spatial layouts and then learn weighted spatial layouts by an $\ell_{1}$ regularized max-margin optimization problem for scene recognition. Our proposed joint learning of class-specific spatial layouts and image classifiers can improve the scene recognition performance compared to existing approaches that do not explicitly exploit the spatial layout information. Experiments on Scene15, Landuse-21 and MIT Indoor-67 datasets validate the advantages of our proposed algorithm. 
There are still potential extensions to our proposed method for scene recognition. For example, currently our model is focusing on images, it will be interesting if we extend our work to videos with both spatial layout and temporal layout to improve the action recognition performance. 



\section{Chapter 4}

\section{Efficient Discovery of AND/OR}

\section{Co-occurrence Patterns for Visual}

\section{Recognition}

\subsection{Introduction}

The development of various visual features provides us more options to describe images and videos $[32,74,87]$. However, an individual feature modality, e.g. color or texture, could be too weak to achieve satisfactory result due to the high complexity of visual objects and scenes. Many previous works have shown that appropriate compositions of individual features or different types of features, namely the co-occurrence feature, can be more discriminative and hence leads to more accurate results in visual recognition and multimedia analysis $\left[3,88_{-}\right.$ 94]. For example, in the work [88] features mining was proposed to minimize the human effort to explore the vast space of possible features for image classification. In [92], compositional object patterns are discovered from personal album images for event recognition. In [94], histograms of high-order pattern sets are proposed to represent images for object recognition.

Despite the success of co-occurrence features in recent progress, mining discriminative cooccurrence features from a large number of candidate features remains a challenging problem. 
The first challenge comes from the huge number of possibilities of composing high-order cooccurrence features. Given $N$ binary features, in total we can obtain a pool of $2^{N}$ (or $3^{N}$ if considering both positive and negative responses) co-occurrence features [3], from which it is a computational demanding task to seek the one with the minimal training error. In practical tasks, the value of $N$ could be thousands or even larger, and it is computationally intractable to perform an exhaustive search in the exponentially large number of candidates. A detailed co-occurrence feature mining survey can be found in [95].

To reduce the exhaustive search time, some existing approaches propose to search for fixedorder co-occurrence features $[28,81]$, or use the sequential forward selection algorithm [89, 96]. However, these approaches cannot guarantee the optimality of the discovered co-occurrence features, hence resulting in less effective features. The recent work [3] has proposed a two-stage frequent pattern mining method to select the optimal co-occurrence features for boosting. In the first stage it discovers the candidates of optimal co-occurrence features with mild frequency. Then it selects the optimal co-occurrence feature with minimum training error in the second stage. These co-occurrence features are finally boosted together to form a strong classifier. Such a two-stage feature discovery method, although can effectively find and combine discriminative co-occurrence features, is still not affordable when the feature pool is of large size. In such a case, the candidate co-occurrence feature of mild frequency can be of a huge number, thus it is not feasible to check all candidates to pick the optimal one.

Besides, after the discriminative co-occurrence features are discovered, how to integrate them appropriately into an effective classifier also needs to be studied. Due to the large intra-class variations of object, scene and action categories, one single co-occurrence feature is usually not enough to describe one category. Boosting is one common way to integrate the co-occurrence features $[3,97,98]$.

This chapter addresses the limitations of [3] by proposing a direct branch-and-bound search based method for co-occurrence feature mining. We enrich the original set of binary features by adding their complements such that AND/OR co-occurrence features can be simultaneously mined by frequent pattern mining algorithms. Different from the two-stage feature mining 
method in [3], our method can mine both AND/OR co-occurrence features directly in one stage, and the designed branch-and-bound search can efficiently mine the optimal AND/OR co-occurrence feature from the weighted training dataset. Thus our proposed method can be order of magnitude faster. We use Adaboost.MH algorithm [99] (the multi-class extension of Adaboost) to combine the discovered co-occurrence features to achieve better generalization error for multi-class classification problems. Experiments on several publicly available datasets, e.g., the benchmark datasets from UCI repository, the Scene-15 dataset, and the MSR Daily Activity 3D dataset validate both the effectiveness and efficiency of our proposed method.

The rest of this chapter is organized as follows. In Sec. 4.2 we have a literature review. In Sec. 4.3 we introduce our proposed optimal AND/OR pattern mining for boosting method. In Sec. 4.4 we conduct several experiments on UCI machine learning datasets, the Scene-15 dataset and the MSR Daily Activity 3D dataset. In Sec. 4.5 we have a conclusion.

\subsection{Related Work}

Adaboost [100] is one of the best known ensemble learning methods that can build a highly accurate classifier by using only simple binary base learners. It was later extended to the multiclass version, AdaBoost.MH [99], to solve multi-class classification problems.

However boosting with simple base classifier such as decision stumps has often been found sub-optimal. As discussed in [101], the linear combinations of decision stumps cannot be a universal approximator. For example, the XOR problem cannot be addressed by linear combination of decision stumps. The common solution to overcome this problem is using decision trees which are learnt in a greedy recursive fashion.

The weakness of individual visual features drives us to look for the appropriate combinations of them which are of more discriminative power in object detection, image classification and action recognition $[88,96,102]$. Many previous works have focused on generating stronger compositional features, such as visual grouplet [103], visual phrase [26, 104, 105] and cooccurrence feature $[3,81]$. 
The work in $[28,81]$ make use of the spatial information to enhance the discriminative ability of compositional features. However, it leads to much higher complexity and hence only the second-order compositional feature is considered. In [106, 107], only the conjunction form (AND) of subgraph and subsequence are considered for image and video analysis.

Recently in [93], frequent local histogram (FLH) features are mined as mid-level features and then bag-of-FLHs image representations are formed for image classification applications. Similarly in the work of [94], Winn et al. proposed to first mine frequent pattern sets using Frequent Pattern Mining method and then represent each image by histogram of the mined pattern set for object recognition problems. Both these methods had used the discovered compositional features as mid-level features instead of the traditional raw features such as the Local Binary Patterns (LBP) [108], Histogram of Gradients (HOG) [109], or the Bag-of-Words (BoW) [25].

In order to enrich the variety of co-occurrence features, Yuan et al. proposed to discover both the conjunction (AND) and disjunction (OR) of individual features by a two-round two-stage method in [3]. In the first round they mined the discriminative AND patterns, after that they flipped the sample labels and then mined the discriminative OR patterns in the second round. For each round, they employed a two-stage method by first generating a set of AND/OR pattern candidates using Frequent Pattern Mining algorithm and then exhaustively searching over the candidate pool to discover the discriminative AND/OR pattern.

In spite of the introduction of both conjunction and disjunction patterns in the previous compositional feature mining work, how to efficiently mine the optimal co-occurrence feature with minimal training error still remains a challenging problem. Many research work such as $[89,96]$ use the sequential forward selection method to avoid exhaustive search but cannot guarantee optimality of the discovered co-occurrence features. The necessary conditions of the optimal co-occurrence features are provided in [3], under which the number of the candidates will decrease and thus an efficient search can be performed. However, it is still computationally expensive because the necessary conditions are pre-defined and usually not tight enough.

In the actionlet ensemble work [10], Wang et al. proposed to mine a set of discriminative actionlets (which are defined as conjunction form of base skeleton features) and then apply a 
multiple kernel learning approach to combine them for action recognition applications. The main differences of the pattern mining methods between ours and the actionlet ensemble work are two-fold: 1) our method can mine both conjunction form (AND pattern) and disjunction form (OR pattern) of base features, while the method in actionlet ensemble work only mines the actionlet in conjunction form; 2) our method can mine the optimal AND/OR pattern with minimal training error and thus are suited for the boosting framework, while the actionlet ensemble work only employs an heuristic method to select discriminative actionlets and thus cannot guarantee optimal solution.

\subsection{Proposed Method}

\subsubsection{Overview}

We show the overview of our proposed efficiently mining optimal AND/OR patterns for boosting method in Fig. 4.1. As can be seen from the figure, our method contains the following 5 steps: (a) at each boosting iteration, we are given the labeled and weighted real-valued image/video features; (b) we binarize the real-valued features; (c) we construct transactions according to the binarized features for further data mining analysis; (d) we mine the optimal AND/OR pattern from the transactions using our proposed branch-and-bound search method; (e) we use the discovered AND/OR pattern as weak learner in the boosting framework. Details of each step will be discussed in the following sections.

\subsubsection{Feature Binarization}

We start from considering binary classification problem since it can be naturally extended to multi-class classification problem by the Adaboost.MH algorithm [99].

Let the training dataset be $\mathbf{D}=\left\{\left(\mathbf{x}_{i}, y_{i}, w_{i}\right)\right\}_{i=1}^{N}$, where each $\mathbf{x}_{i} \in \mathbb{R}^{d}$ denotes the sample feature vector, $y_{i} \in\{-1,+1\}$ is the category label and $w_{i}$ is the weight of sample $x_{i}$ and $\sum_{i} w_{i}=1$. 


\begin{tabular}{|c|c|c|c|c|c|c|}
\hline$y_{i}$ & $w_{i}$ & $x_{i}$ & \multirow{4}{*}{$\Rightarrow$} & $x_{i}$ & \multirow{4}{*}{$\Longrightarrow$} & $x_{i}$ \\
\hline-1 & 0.4 & $\{0.5,0.1 \ldots 0.2\}$ & & $\{+1,-1 \ldots-1\}$ & & $\left\{e_{1}, \overline{e_{2}} \ldots \overline{e_{\mathrm{T}}}\right\}$ \\
\hline-1 & 0.2 & $\{0.7,0.2 \ldots 0.3\}$ & & $\{+1,-1 \ldots+1\}$ & & $\left\{e_{1}, \overline{e_{2}} \ldots e_{T}\right\}$ \\
\hline+1 & 0.3 & $\{0.6,0.4 \ldots 0.4\}$ & & $\{+1,+1 \ldots+1\}$ & & $\left\{e_{1}, e_{2} \ldots e_{T}\right\}$ \\
\hline+1 & 0.1 & $\{0.4,0.3 \ldots 0.1\}$ & & $\{-1,-1 \ldots-1\}$ & & $\left\{\overline{e_{1}}, \overline{e_{2}} \ldots \overline{e_{\mathrm{T}}}\right\}$ \\
\hline
\end{tabular}

a) Labeled and weighted dataset

(b) Feature binarization

(c) Induced transactions

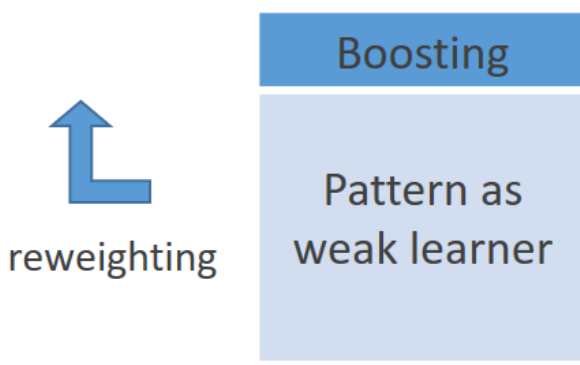

(e) Weak learner

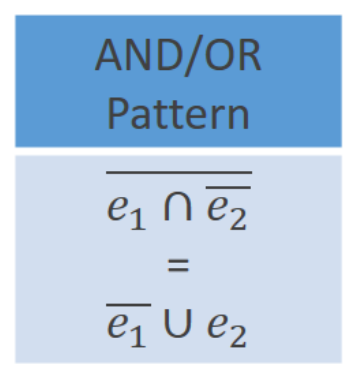

(d) AND/OR pattern

FIGURE 4.1. Overview of the proposed efficiently mining optimal AND/OR patterns for boosting method.

Instead of using the real-valued raw feature vector $\mathrm{x}$ directly, we transform it into binary vectors by a collection of binary functions $\left\{\mathbf{h}_{1}, \mathbf{h}_{2}, \ldots, \mathbf{h}_{T}\right\}$. For example, each $\mathbf{h}_{t}$ can be defined as a decision stump,

$$
\mathbf{h}_{t}(\mathbf{x})= \begin{cases}e_{t} & \text { if } \mathbf{x}\left(j_{t}\right) \geq \theta_{t} \\ \overline{e_{t}} & \text { otherwise }\end{cases}
$$

where $\mathbf{x}\left(j_{t}\right)$ is the value of $\mathbf{x}$ in the $j_{t}$ dimension, and $\theta_{t}$ is the quantization threshold and $e_{t}, \overline{e_{t}} \in\{-1,+1\}$.

There are other binarization methods, such as the random projected decision stumps as follows,

$$
\mathbf{h}_{t}(\mathbf{x})= \begin{cases}e_{t} & \text { if } \mathbf{v}_{t} \cdot \mathbf{x} \geq \theta_{t} \\ \overline{e_{t}} & \text { otherwise }\end{cases}
$$

where $\mathbf{v}_{t}$ is a randomly generated vector. 
In order to generate a set of decision stumps (or random projected decision stumps) from raw samples for feature binarization, we exhaustively search the optimal threshold at each dimension of the raw feature (or the randomly projected feature) according to the minimum training error $\epsilon(\mathbf{h})$,

$$
\arg \min _{\mathbf{h}} \epsilon(\mathbf{h})=\sum_{i} w_{i} I\left(\mathbf{h}\left(\mathbf{x}_{i}\right) \neq y_{i}\right)
$$

where $I(\cdot)$ is the indicator function.

\subsubsection{Induced Transaction}

After the feature binarization step, we have constructed an item vocabulary as $\Omega=\left\{e_{1}, \ldots, e_{T}, \overline{e_{1}}, \ldots, \overline{e_{T}}\right\}$. We can randomly now transform each training sample $\mathrm{x}$ into a transaction according to its responses of the $T$ feature primitives:

$$
\mathcal{T}(\mathbf{x})=\left\{\mathbf{h}_{1}(\mathbf{x}), \mathbf{h}_{2}(\mathbf{x}), \ldots, \mathbf{h}_{T}(\mathbf{x})\right\} \subset \Omega
$$

Then the original dataset $\mathbf{D}$ is transferred into a transaction dataset $\mathbf{T}=\left\{\left(\mathcal{T}\left(\mathbf{x}_{i}\right), y_{i}, w_{i}\right)\right\}_{i=1}^{N}$, which is illustrated in Fig. 4.2. By transforming continuous feature $\mathbf{x}_{i}$ into discrete transaction $\mathcal{T}\left(\mathbf{x}_{i}\right)$, we essentially describe each training sample by $T$ discrete items such that the composition feature mining problem can be solved by the traditional Frequent Pattern Mining algorithms.

It is worth noting when constructing the transaction, we have used both the positive response and negative response $\left(e_{t}\right.$ and $\overline{e_{t}}$ ) of the binary function $\mathbf{h}_{t}$ as items of the transaction. It is important that we also include the negative responses, otherwise only the conjunction form of the individual features can be discovered from the induced transaction dataset.

\subsubsection{Mining Optimal AND/OR Patterns}

After the feature binarization and transaction construction steps, in this section we will introduce our co-occurrence feature mining method. 


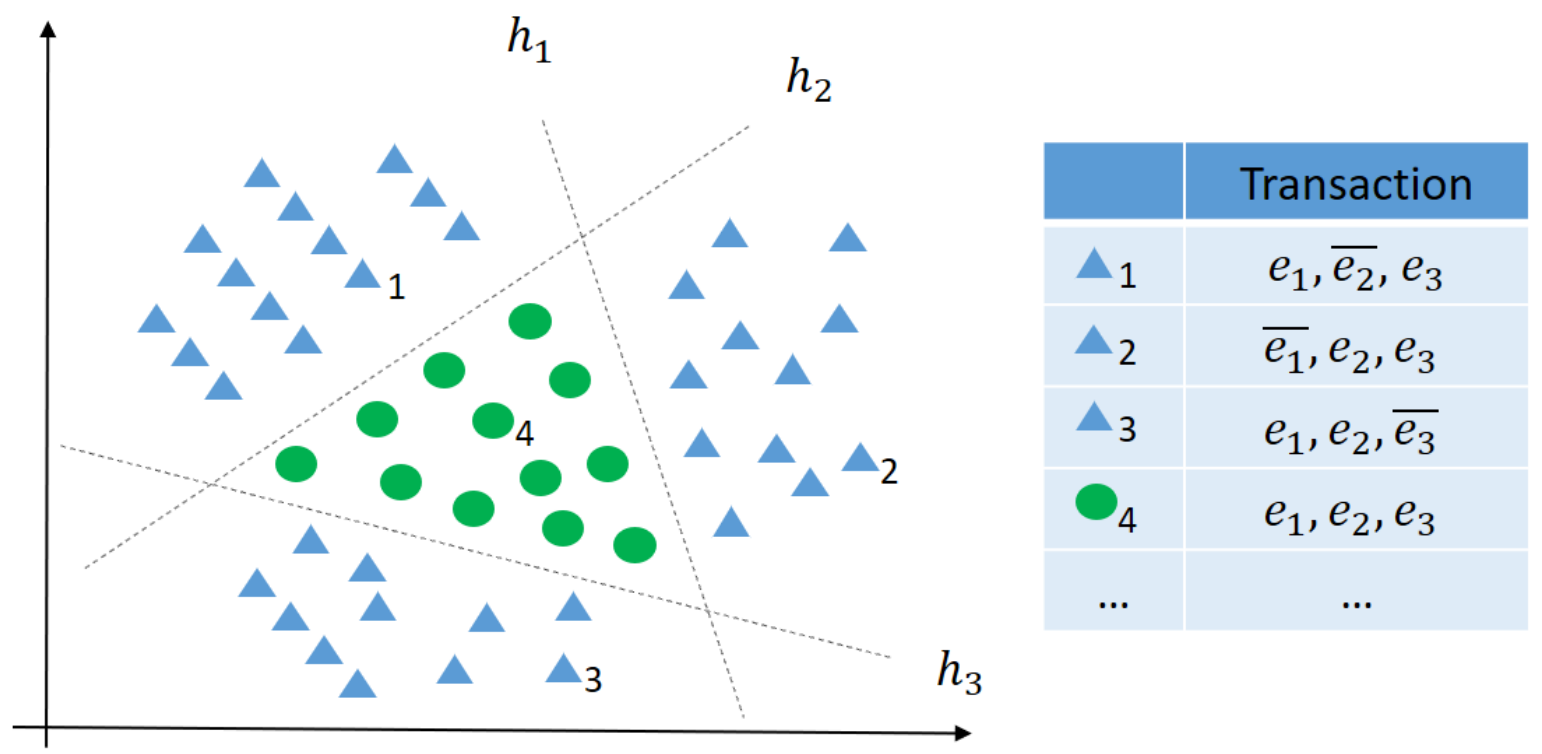

FIGURE 4.2. Illustration of the induced transaction using random projected decision stumps on $2 \mathrm{D}$ points.

We define the co-occurrence features as both conjunction form (AND pattern) and disjunction form (OR pattern) of individual features of arbitrary order as follows,

$$
P(\mathbf{x}) \equiv\left\{\begin{array}{cl}
\mathbf{h}_{i} \wedge \mathbf{h}_{j} \wedge \cdots \wedge \mathbf{h}_{k}(\mathbf{x}) & \text { AND pattern } \\
\mathbf{h}_{i} \bigvee \mathbf{h}_{j} \bigvee \cdots \bigvee \mathbf{h}_{k}(\mathbf{x}) & \text { OR pattern }
\end{array}\right.
$$

where each $\mathbf{h}_{i}$ can be either $e_{i}$ or $\overline{e_{i}}$, as defined in Eq. 4.1 and 4.2.

It is worth noting that OR patterns can be converted into the complementary forms of AND patterns. For example, according to De Morgan's laws, i.e., $\mathbf{h}_{i} \bigvee \mathbf{h}_{j} \bigvee \cdots \bigvee \mathbf{h}_{k}=\overline{\overline{\mathbf{h}_{i}} \bigwedge \overline{\mathbf{h}_{j}} \wedge \cdots \wedge \overline{\mathbf{h}_{k}}}$, we can transform the problem of mining OR patterns into mining the complementary forms of AND patterns, which is a much easier problem to solve using the Frequent Pattern Mining methods.

The optimal AND/OR pattern is defined as the one with the minimal training error $\epsilon(P)$ as follows,

$$
\arg \min _{P} \epsilon(P)=\sum_{i} w_{i} I\left(P\left(\mathbf{x}_{i}\right) \neq y_{i}\right)
$$

Therefore in order to find the optimal AND/OR pattern, we can exhaustively calculated the 
training errors of all AND patterns and their complementary forms from the transaction dataset constructed by Eq. 4.4, and then select the one with the minimal training error.

However such an exhaustive search is too time-consuming. In the following section we will introduce an efficient branch-and-bound search based algorithm to mine the optimal AND/OR pattern from the transaction dataset.

\subsubsection{Efficiently Mining Optimal AND/OR Patterns}

Before discussing the branch-and-bound algorithm, let us declare some notations first. Given a training dataset $\mathbf{T}=\left\{\left(\mathcal{T}\left(\mathbf{x}_{i}\right), y_{i}, w_{i}\right)\right\}_{i=1}^{N}$, we can calculate total weight of positive samples $r^{+}$ and total weight of negative class $r^{-}$:

$$
r^{+}=\sum_{i: y_{i}=+1} w_{i} \quad \text { and } \quad r^{-}=\sum_{i: y_{i}=-1} w_{i}
$$

Note that $r^{+}+r^{-}=\sum_{i} w_{i}=1$ because weights for the dataset are pre-normalized.

Moreover, we can calculate the weighted frequency of pattern $P$ in positive and negative classes respectively:

$$
f^{+}=\sum_{\substack{i: y_{i}=+1, P \subseteq \mathcal{T}\left(\mathbf{x}_{i}\right)}} w_{i} \quad \text { and } \quad f^{-}=\sum_{\substack{i: y_{i}=-1, P \subseteq \mathcal{T}\left(\mathbf{x}_{i}\right)}} w_{i}
$$

Then the pattern frequency $f(P)$ in the whole training dataset is given by:

$$
f(P)=\sum_{i: P \subseteq \mathcal{T}\left(\mathbf{x}_{i}\right)} w_{i}=f^{+}+f^{-}
$$

\subsubsection{Pattern Frequency Constraints}

It is not hard to see that a discriminative AND/OR pattern should satisfy the mild-frequency requirements, i.e., its frequency $f(P)$ should not be too high or too low. The reason is that a pattern of high frequency is likely to appear in both positive and negative samples, thus leads to 
a low precision; on the other hand, a pattern of low frequency cannot cover the whole positive class and thus leads to a low recall.

We now show that in order to mine the optimal AND/OR pattern with the minimal training error, we do not need to exhaustively search over all the possible pattern candidates since there are frequency constraints for the desired AND/OR pattern, which means, an efficient Frequent Pattern Mining method can be applied.

A discriminative AND/OR pattern can be either used to predict the positive class or to predict the negative class samples, therefore we use the following criteria to choose the optimal AND/OR pattern $\hat{P}$,

$$
\hat{P}= \begin{cases}P & \text { if } \epsilon(P) \leq \epsilon(\bar{P}) \\ \bar{P} & \text { otherwise }\end{cases}
$$

On the one hand, when we use pattern $P$ to predict positive class, the training error $\epsilon(P)$ can be calculated as follows,

$$
\begin{array}{r}
\epsilon(P)=r^{+}-f^{+}+f^{-} \\
\text {s.t. } \quad 0 \leq f^{+} \leq r^{+}
\end{array}
$$

Then given an error tolerance parameter $\mu$ such that $\epsilon(P) \leq \mu$, we will have the following frequency constraints for the target AND pattern,

$$
\begin{gathered}
f(P)=f^{+}+f^{-} \leq r^{+}+f^{-} \leq f^{+}+\mu \leq r^{+}+\mu \\
f(P)=f^{+}+f^{-} \geq f^{+}-f^{-} \geq r^{+}-\mu
\end{gathered}
$$

That is,

$$
r^{+}-\mu \leq f(P) \leq r^{+}+\mu
$$


On the other hand, when we use the complementary form $\bar{P}$ to predict positive class (or in other words, we use $P$ to predict negative class), we can calculate the training error $\epsilon(\bar{P})$ as follows,

$$
\begin{array}{r}
\epsilon(\bar{P})=r^{-}-f^{-}+f^{+} \\
\text {s.t. } \quad 0 \leq f^{-} \leq r^{-}
\end{array}
$$

Then similarly we can derive the following inequality,

$$
r^{-}-\mu \leq f(\bar{P}) \leq r^{-}+\mu
$$

Since the optimal pattern $\hat{P}$ can be either $P$ or $\bar{P}$, we have the following pattern frequency constraints for the optimal AND/OR pattern,

$$
\begin{array}{r}
\xi_{1}(\mu) \leq f(\hat{P}) \leq \xi_{2}(\mu) \\
\xi_{1}(\mu)=\min \left(r^{+}-\mu, r^{-}-\mu\right) \\
\xi_{2}(\mu)=\max \left(r^{+}+\mu, r^{-}+\mu\right)
\end{array}
$$

where $\xi_{1}(\mu)$ is the lower bound and $\xi_{2}(\mu)$ is the upper bound of the pattern frequency $f(\hat{P})$ given the parameter $\mu$, respectively.

From Eq. 4.15 we can see that using the pattern frequency constraints we can reject too frequent and unfrequent patterns directly and hence speed up the exhaustive pattern search. However, since the parameter $\mu$ is pre-defined and fixed, the constraints are still not tight enough and can lead to poor speed performance. In the following section we will introduce our branch-andbound search based solution to further speed up the mining process.

\subsubsection{Branch-and-Bound Search}

In order to discover the optimal AND/OR pattern, we seek the minimal training error from Eq. 4.11 and 4.13. Recall that $\min (a, b)=[(a+b)-|a-b|] / 2$, we can obtain the following 
equation,

$$
\begin{aligned}
\epsilon(\hat{P}) & =\min \left(r^{+}-f^{+}+f^{-}, r^{-}-f^{-}+f^{+}\right) \\
& =\frac{1}{2}\left(r^{+}+r^{-}\right)-\frac{1}{2}\left|\left(r^{+}-r^{-}\right)-2\left(f^{+}-f^{-}\right)\right|
\end{aligned}
$$

We denote $\gamma(\hat{P})=\left|\left(r^{+}-r^{-}\right)-2\left(f^{+}-f^{-}\right)\right|$then we can derive the following inequality,

$$
\begin{aligned}
\gamma(\hat{P}) & \leq\left|r^{+}-r^{-}\right|+2 \cdot\left|f^{+}-f^{-}\right| \\
& \leq\left|r^{+}-r^{-}\right|+2 \cdot \max \left(f^{+}, f^{-}\right)
\end{aligned}
$$

Therefore we have the lower bound $\tau(\hat{P})$ for the training error $\epsilon(\hat{P})$ as follows,

$$
\begin{aligned}
\epsilon(\hat{P}) & \geq \frac{1}{2}\left(r^{+}+r^{-}\right)-\frac{1}{2}\left|r^{+}-r^{-}\right|-\max \left(f^{+}, f^{-}\right) \\
& =\text {const }-\max \left(f^{+}, f^{-}\right)=\tau(\hat{P})
\end{aligned}
$$

Using this lower bound $\tau(\hat{P})$, we can now design a branch-and-bound search based method to directly mine the optimal AND/OR pattern on the conditional FP-Trees that are recursively constructed by the FP-Growth algorithm [13]. Due to space limitation, we refer the readers to [13] for the details of the classical FP-Growth algorithm.

Within a given conditional FP-Tree, we assume all the possible candidate patterns are denoted as $\left\{\hat{P}_{n} \mid n \in\{1, \cdots N\}\right\}$, and $\hat{P}_{1}$ is the root pattern of this tree. We represent the training error of pattern $\hat{P}_{n}$ as $\epsilon\left(\hat{P}_{n}\right)$, and the error lower bound as $\tau\left(\hat{P}_{n}\right)$. First, according to the definition of the upper bound, we have $\epsilon\left(\hat{P}_{n}\right) \geq \tau\left(\hat{P}_{n}\right)$. Second, since the FP-Tree is a frequency prefix tree [13], then the pattern frequencies $f^{-}, f^{+}$are non-decreasing along the leave-to-root path of the FP-Tree, and thus $\tau\left(\hat{P}_{n}\right)=$ const $-\max \left(f^{-}, f^{+}\right)$can achieve the maximal value at the root, i.e., we have $\tau\left(\hat{P}_{n}\right) \geq \tau\left(\hat{P}_{1}\right)$. Third, if we have found that $\tau\left(\hat{P}_{1}\right)>\epsilon\left(\hat{P}^{*}\right)$ where $\hat{P}^{*}$ is the 
previous best discovered pattern, then we can easily derive that

$$
\epsilon\left(\hat{P}_{n}\right) \geq \tau\left(\hat{P}_{n}\right) \geq \tau\left(\hat{P}_{1}\right)>\epsilon\left(\hat{P}^{*}\right)
$$

which means, any candidate pattern $\hat{P}_{n}$ has no smaller training error than the previous best pattern $\hat{P}^{*}$, therefore we can safely ignore all the possible candidate patterns from this conditional FP-Tree.

The basic branch-and-bound idea is, in the recursive FP-Growth mining process, we record the current best pattern $\hat{P}^{*}$ and its corresponding error $\epsilon\left(\hat{P}^{*}\right)$ using global variables. Before proceeding a new conditional FP-Tree, we estimate the error lower bound $\tau\left(\hat{P}_{1}\right)$ of the root pattern $\hat{P}_{1}$ by Eq. 4.20. If the lower bound value $\tau\left(\hat{P}_{1}\right)$ of this new conditional FP-Tree is no smaller than the current best $\epsilon\left(\hat{P}^{*}\right)$, i.e., $\tau\left(\hat{P}_{1}\right) \geq \epsilon\left(\hat{P}^{*}\right)$, then we can safely ignore this conditional FP-Tree as well as any other conditional FP-Trees recursively constructed from this one.

It is also worth noting that, during the branch-and-bound search method stated above, we can relax the tolerance parameter $\mu$, and thus update the pattern frequency constraints discussed in Sec. 4.3.5.1, i.e., update the pattern frequency lower bound $\xi_{1}$ and upper bound $\xi_{2}$, to help speed up the patten mining process.

During the pattern mining process, we denote the latest two discovered patterns as $\hat{P}_{\text {new }}$ and $\hat{P}_{\text {old }}$, their corresponding training errors as $\epsilon\left(\hat{P}_{\text {new }}\right)$ and $\epsilon\left(\hat{P}_{\text {old }}\right)$, and the corresponding tolerance parameter as $\mu_{\text {new }}$ and $\mu_{\text {old }}$. From $\hat{P}_{\text {old }}$ to $\hat{P}_{\text {new }}$ we can compute the decrement of error as $\Delta \epsilon=\epsilon\left(\hat{P}_{\text {new }}\right)-\epsilon\left(\hat{P}_{\text {old }}\right)<0$. Since we have assumed that $\epsilon\left(\hat{P}_{\text {old }}\right) \leq \mu_{\text {old }}$, we can now use $\mu_{\text {new }}=\mu_{\text {old }}+\Delta \epsilon$ to make the inequality $\epsilon\left(\hat{P}_{\text {new }}\right) \leq \mu_{\text {new }}$ hold, i.e., the tolerance parameter $\mu$ can be updated from $\mu_{\text {old }}$ to a smaller value $\mu_{\text {new }}$. Then according to Eq. 4.16 and 4.17 we can update $\xi_{1}$ and $\xi_{2}$ as follows,

$$
\begin{aligned}
& \xi_{1}\left(\mu_{\text {new }}\right)=\xi_{1}\left(\mu_{\text {old }}\right)-\Delta \epsilon \\
& \xi_{2}\left(\mu_{\text {new }}\right)=\xi_{2}\left(\mu_{\text {old }}\right)+\Delta \epsilon
\end{aligned}
$$


That is, as the branch-and-bound search goes on, pattern frequency constraints will become tighter and tighter, and thus more and more conditional FP-Trees can be discarded using the tighter frequency constraints.

The overview of proposed branch-and-bound search based optimal AND/OR pattern mining algorithm is shown in Alg. 5. The main algorithm flow is similar to FP-Growth: The main procedure OPMine $(\cdot)$ builds FP-Tree and then calls the recursive procedure BBMine $(\cdot)$, where we perform branch-and-bound search on recursively generated conditional FP-Trees.

In Alg. 5, we use 4 global variables to keep track of the optimal AND/OR pattern, i.e., current best AND/OR pattern $\hat{P}^{*}$, its corresponding error $\epsilon^{*}$, current pattern frequency lower bound $\xi_{1}^{*}$ and pattern frequency upper bounder $\xi_{2}^{*}$. Line 12 shows the main branch-and-bound search method discussed in Sec. 4.3.5.2. Line 6 and 32 show the pattern frequency constraints discussed in Sec. 4.3.5.1. In line 20-23 we first update the optimal AND/OR pattern $\hat{P}^{*}$, and then its corresponding training error $\epsilon^{*}$ according to Eq. 4.18, and then the pattern frequency lower bound $\xi_{1}^{*}$ by Eq. 4.22 and the upper bound $\xi_{2}^{*}$ by Eq. 4.23 .

\subsubsection{Boosting with Mined Optimal AND/OR Patterns}

After mining the optimal AND/OR pattern with minimal training error, we will integrate the pattern into the multi-class boosting algorithm Adaboost.MH [99] to obtain better generalization ability. The pseudo code of the proposed boosting with optimal AND/OR patterns algorithm is shown in Alg. 6, where we follow the basic procedure of the Adaboost.MH algorithm for multi-class classification.

In Alg. 6, the feature binarization step is shown in line 3, where we determine the thresholds of the decision stumps by exhaustive search at each dimension of the raw feature vector as discussed in Sec. 4.3.2. The transaction generation step is shown in line 4 as discussed in Sec. 4.3.3. The optimal AND/OR pattern mining via branch-and-bound search is shown in line 6 as discussed in Sec. 4.3.5. 


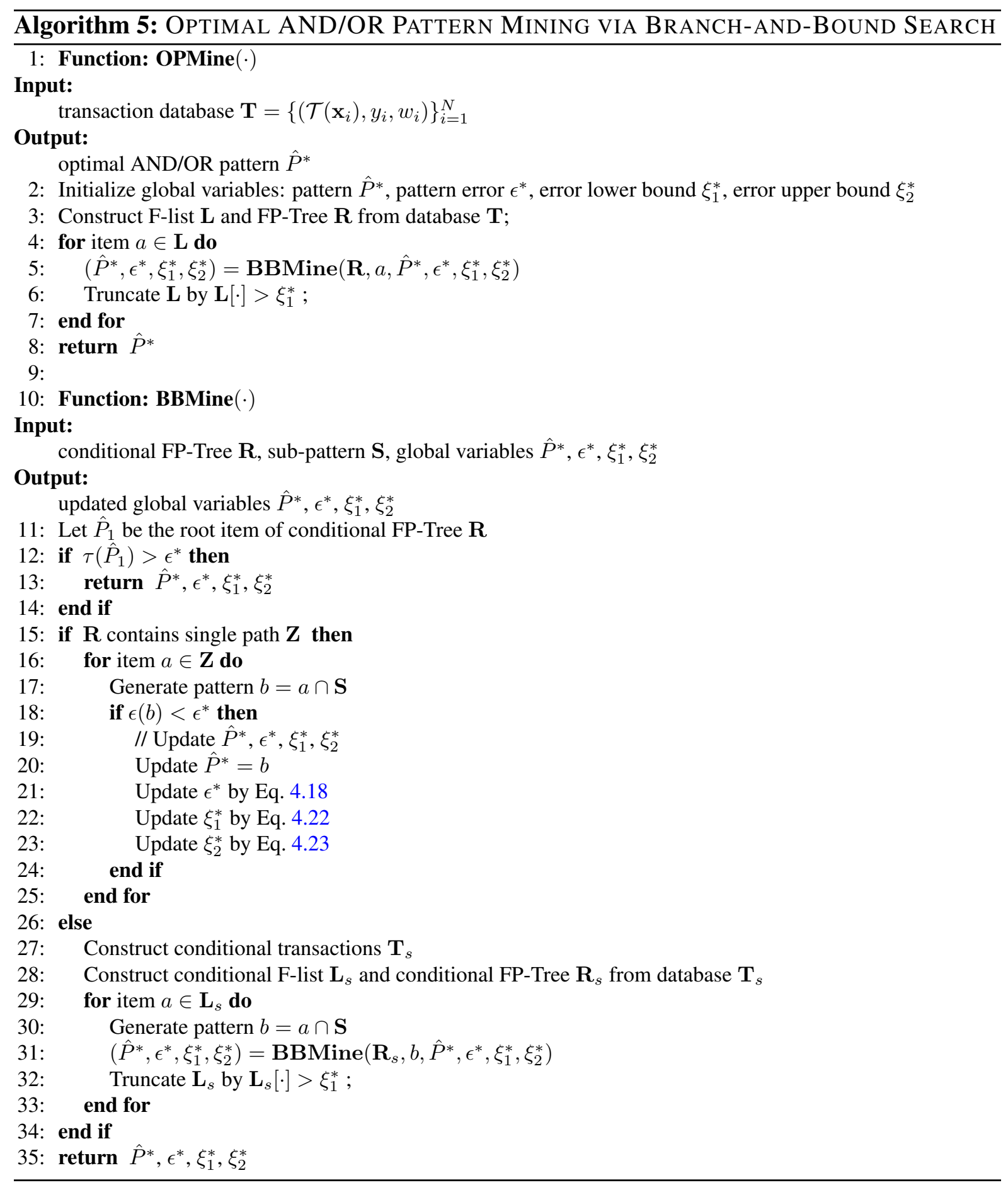

\subsection{Experiments}

In this section, we report our experimental results on several publicly available datasets for different tasks. First we conduct our experiments on some benchmark datasets including the 


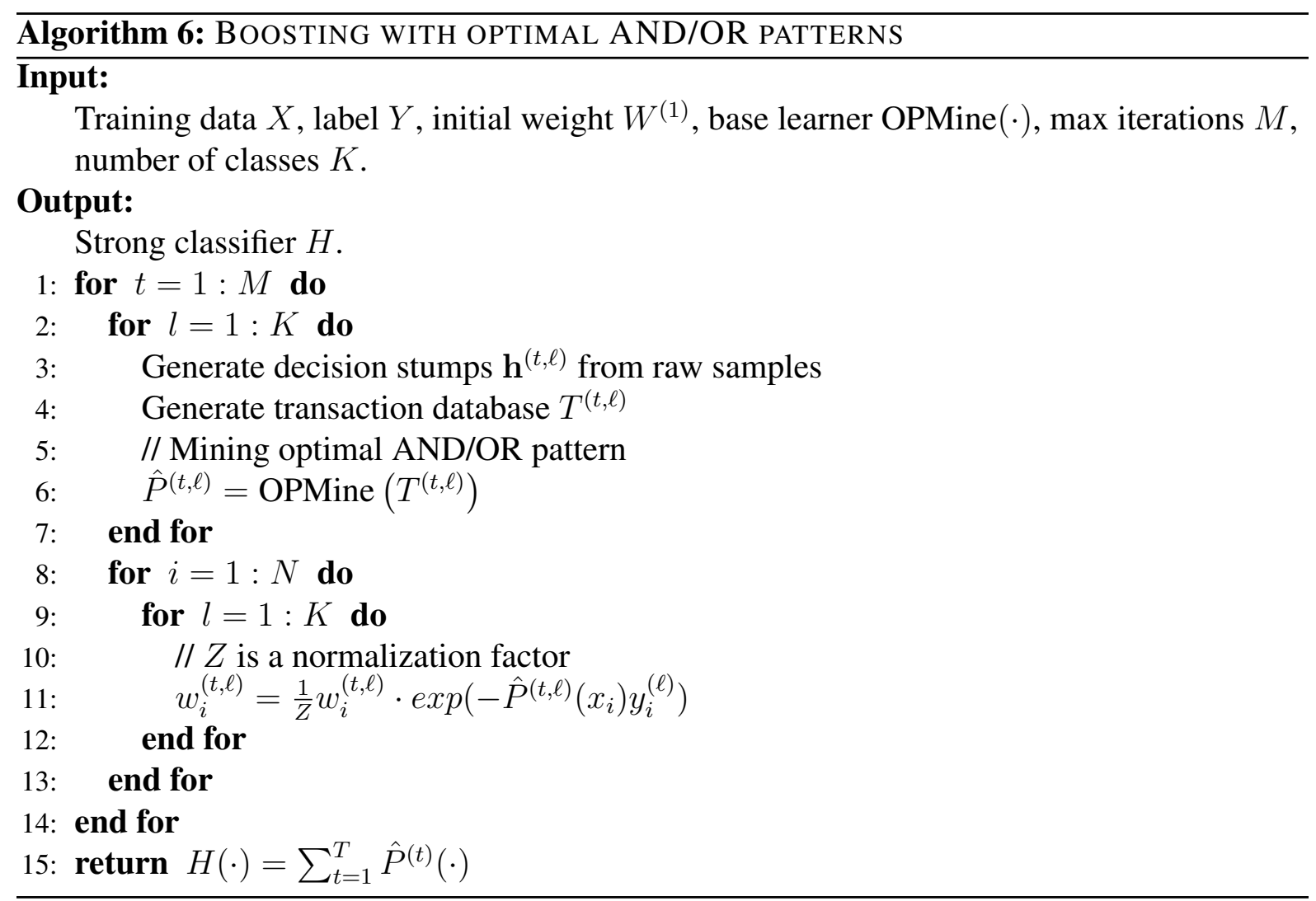

$\mathrm{UCI}^{1}$ machine learning datasets, the USPS ${ }^{2}$ digits dataset and the MNIST ${ }^{3}$ digits dataset to validate 1) the effectiveness of combining boosting algorithm with AND/OR patterns; 2) the efficiency of our proposed branch-and-bound search based optimal AND/OR pattern mining method. Second, we conduct our scene recognition experiments on the Scene-15 dataset 4 and achieve the state-of-the-art performance. Third, we also test our optimal AND/OR pattern mining algorithm for action recognition problems on the MSR Daily Activity 3D ${ }^{5}$ dataset.

\footnotetext{
${ }^{1}$ UCI. http://archive.ics.uci.edu/ml/

${ }^{2}$ USPS. http://www.cs.nyu.edu/ roweis/data.html

${ }^{3}$ MNIST. http://yann.lecun.com/exdb/mnist

${ }^{4}$ Scene-15 Dataset. http://www-cvr.ai.uiuc.edu/ponce-grp/data/

${ }^{5}$ MSR Daily Activity 3D Dataset. http://research.microsoft.com/en-us/um/people/zliu/ActionRecoRsrc/
} 


\subsubsection{Benchmark Datasets}

\subsubsection{Effectiveness Test}

In this section, we evaluate the discriminative power of the discovered AND/OR patterns on 16 publicly available benchmark datasets. Table 4.1 shows the basic dataset information.

In the experiments, for those datasets with standard train/test split, we use the original settings. For those datasets without train/test split, we perform 10-fold cross-validation. We compare 3 different base classifiers for boosting: 1) decision stump; 2) decision tree; and 3) AND/OR pattern. For the decision tree experiments, we limit the maximum of tree leaves number to 8 (we tried different values for this parameter and found this one is fair enough for different datasets). For our AND/OR patterns, we use decision stumps on each dimension of the raw features for feature binarization and then based on these decision stumps we mine our optimal AND/OR patterns for boosting. For all the experiments, we run the boosting algorithms until it converges on each dataset.

The experimental results are shown in Table 4.2 , in which all the numbers are in $\%$. The $2^{\text {nd }}$ to $4^{\text {th }}$ columns show the performances of the base classifiers, and the $5^{\text {th }}$ to $7^{\text {th }}$ columns show the performances of the boosted classifiers. The best result of each dataset is shown in bold font.

From the $2^{\text {nd }}$ to $4^{\text {th }}$ columns we can see that one single decision stump or AND/OR pattern is not able to achieve a high accuracy result, which means they do not have enough capacity to be used as stand-alone strong classifiers in practice. On contrary, single decision tree as a "strong" base classifier, achieves the best results among the 3 base classifiers.

From the $5^{\text {th }}$ to $7^{\text {th }}$ columns we can see that when boosting up, the performance improvements of the 2 "weak" base classifiers, i.e., decision stump and AND/OR pattern, are much larger than the "strong" decision tree classifier: Boosting+Stump algorithm improves $40.8 \%$ and Boosting+Pattern 38.6\%, while Boosting+Tree only improves 4.6\%. On average, Boosting+Pattern outperforms the other two types of boosting approaches: among the 16 datasets, Boosting+Pattern achieves 11 best results and 1 equally best result with Boosting+Tree on the iris dataset. 
From the comparing results between decision stump and AND/OR pattern in Table 4.2 we can see that, decision stump has low discriminative power such that it cannot produce strong enough boosted classifier than AND/OR patterns. In Fig. 4.3 we show the training/testing errors over iterations of the Boosting+Stump and Boosting+Pattern algorithms on the USPS and MNIST datasets. From the figure we can see that as we increase the boosting iterations, the Boosting+Pattern algorithm can achieve both smaller training errors and smaller testing errors than the Boosting+Stump algorithm, which suggests that the AND/OR patterns can not only be more discriminative than the decision stumps in the training process, but also have better generalization ability during the testing process.

From the experimental results of AND/OR pattern and decision tree in Table 4.2 we can see that, decision tree can be too powerful and thus it can be prone to overfitting on some datasets when boosting up with these "strong" decision trees. In comparison, although single AND/OR pattern may not be as discriminative as single decision tree, it is also less likely to be overfitting while combined with the boosting framework.

Therefore in summarization, as a tradeoff between the lower discriminative decision stump and the higher-likely overfitting decision tree, the AND/OR pattern can be more effective when combined with the boosting algorithm.

\subsubsection{Efficiency Test}

In this section, we study the efficiency of the proposed branch-and-bound search based optimal AND/OR pattern mining algorithm. We conduct the experiments on a PC with $2.7 \mathrm{GHz}$ Intel Core and 4GB RAM.

There exist greedy algorithms that can mine discriminative patterns very fast but cannot guarantee optimal solution, thus we do not intend to compare with these greedy algorithms in this experiment. Instead our direct competitor is the previous two-round two-stage discriminative AND/OR pattern mining method in [3]. In terms of the speed performance, our proposed optimal AND/OR pattern mining method has two main advantages compared with the two-round 
two-stage method in [3]: 1) our method is based on the branch-and-bound design, while [3] adopts a two-stage method that depends on the exhaustive search of Frequent Pattern Mining algorithm in the first stage; 2) our method can directly mine the optimal AND/OR pattern simultaneously in one branch-and-bound search round, while the method in [3] has to mine the discriminative AND/OR pattern separately in two rounds.

Fig. 4.4 plots the running time of the comparing methods as the item vocabulary size varies, on the USPS and MNIST datasets (for each dataset we randomly choose two class samples in the experiments). From the figure we can see that the proposed branch-and-bound search based optimal AND/OR pattern mining method can be orders of magnitude faster than the two-round two-stage discriminative pattern mining method in [3] as the item vocabulary size increases, e.g., our method is 60 times faster than the previous method in [3] when the item vocabulary size is 700 on the MNIST dataset.

In summarization, thanks to the branch-and-bound search design and the simultaneous AND/OR pattern mining scheme, our proposed optimal AND/OR pattern mining method is significantly more efficient than its direct competitor, i.e., the two-round two-stage discriminative pattern mining method in [3].

\subsubsection{Scene Recognition Dataset}

In this section we apply our proposed boosting with optimal AND/OR patterns method on the Scene-15 dataset $[22,63]$ for scene recognition problem.

The Scene-15 dataset is gradually collected by $[22,63]$ and it contains a variety of outdoor and indoor scenes: bedroom, living room, suburb, industrial, kitchen, coast, forest, highway, inside city, mountain, open country, street, tall building, office, and store. There are about 216 to 410 images of size about $300 \times 250$ in each scene category. The total number of images in the dataset is 4485 .

In the experiment, we combine two types of features for scene recognition: 1) a local descriptor based feature LCC [110] and 2) a holistic feature CENTRIST [74]. For the LCC feature, first 


\begin{tabular}{|l|c|c|c|}
\hline Dataset & $\sharp$ class & $\sharp$ instance & $\sharp$ features \\
\hline \hline sonar & 2 & 208 & 60 \\
\hline ionosphere & 2 & 351 & 34 \\
\hline pima & 2 & 768 & 8 \\
\hline parkinson & 2 & 197 & 23 \\
\hline wdbc & 2 & 569 & 32 \\
\hline \hline iris & 3 & 150 & 4 \\
\hline wine & 3 & 178 & 13 \\
\hline glass & 6 & 214 & 10 \\
\hline vertebral & 3 & 310 & 6 \\
\hline vehicle & 4 & 846 & 18 \\
\hline segment & 7 & 2310 & 19 \\
\hline isolet & 26 & $6238 / 1559$ & 617 \\
\hline letter & 26 & $16000 / 4000$ & 16 \\
\hline pendigits & 10 & $7494 / 3498$ & 16 \\
\hline \hline USPS & 10 & $7291 / 2007$ & 256 \\
\hline MNIST & 10 & $60000 / 10000$ & 784 \\
\hline
\end{tabular}

TABLE 4.1. Benchmark datasets information.

\begin{tabular}{|l|c|c|c||c|c|c|}
\hline Dataset & Stump & Pattern & Tree & B+Stump & B+Pattern & B+Tree \\
\hline \hline sonar & 73.1 & 70.6 & 75.6 & 82.3 & $\mathbf{8 5 . 9}$ & 82.6 \\
\hline ionosphere & 82.4 & 89.0 & 90.0 & 91.0 & 94.6 & $\mathbf{9 5 . 2}$ \\
\hline pima & 74.1 & 73.1 & 74.6 & 74.7 & 75.0 & $\mathbf{7 5 . 6}$ \\
\hline parkinson & 82.9 & 82.3 & 87.1 & 91.1 & $\mathbf{9 2 . 3}$ & 91.2 \\
\hline wdbc & 90.1 & 93.3 & 95.1 & 95.4 & $\mathbf{9 7 . 4}$ & 96.1 \\
\hline \hline iris & 62.0 & 61.6 & 95.3 & 93.8 & $\mathbf{9 5 . 3}$ & $\mathbf{9 5 . 3}$ \\
\hline wine & 73.3 & 79.5 & 91.6 & 96.0 & $\mathbf{9 7 . 7}$ & 97.0 \\
\hline glass & 15.5 & 24.6 & 69.1 & 70.9 & $\mathbf{7 5 . 4}$ & 75.2 \\
\hline vertebral & 65.4 & 64.1 & 81.3 & 81.2 & 81.4 & $\mathbf{8 1 . 6}$ \\
\hline vehicle & 15.4 & 24.3 & 73.5 & 76.1 & $\mathbf{7 9 . 9}$ & 76.4 \\
\hline segment & 89.5 & 93.3 & 94.7 & 97.1 & $\mathbf{9 7 . 7}$ & 96.7 \\
\hline isolet & 1.2 & 4.2 & 83.8 & 95.2 & $\mathbf{9 5 . 5}$ & 94.8 \\
\hline letter & 0.8 & 7.8 & 87.9 & 85.0 & 94.0 & $\mathbf{9 6 . 4}$ \\
\hline pendigits & 11.5 & 32.4 & 92.3 & 94.8 & $\mathbf{9 7 . 8}$ & 96.4 \\
\hline \hline USPS & 17.1 & 14.8 & $86.4 \%$ & 93.5 & $\mathbf{9 5 . 0}$ & 93.6 \\
\hline MNIST & 3.3 & 19.9 & 89.3 & 92.5 & $\mathbf{9 7 . 2}$ & 95.5 \\
\hline \hline Average & 47.3 & 52.2 & 85.4 & 88.1 & $\mathbf{9 0 . 8}$ & 90.0 \\
\hline
\end{tabular}

TABLE 4.2. Accuracy results of the benchmark datasets. 
we extract dense SIFT descriptors with 4 patch sizes (i.e. $7 \times 7,16 \times 16,25 \times 25$, and $31 \times 31$ ) every 8 pixels and then learn a codebook of size 4096 using $K$-means clustering algorithm. Then we partition each image into 10 cells, i.e. $1 \times 1$ and $3 \times 3$, to delineate the spatial layout, by following the same idea of the spatial pyramid matching (SPM) [22]. After that, each image is represented by a feature vector of 40960 dimensions. For the CENTRIST feature, first we partition each image into 25 blocks (level 2), 5 blocks (level 1), and 1 block (level 0) respectively, as used in [74]. Then we use principal component analysis (PCA) to reduce the CENTRIST features to the dimension of 40. After that, each image is represented by a 1240dimensional feature vector. Combining these two types of features, each image is represented by a feature vector of $40960+1240=42200$ dimensions.

In the experiment, for efficiency concern we have limited the item vocabulary size to 100 . Then the mining time for each AND/OR pattern is less than 1 second. We use decision stumps at each dimension of the raw features for feature binarization and then mine the optimal AND/OR pattern for each boosting iteration based on these decision stumps. We run the boosting algorithm for 1000 iterations in the experiments.

In terms of the accuracy performance, our proposed optimal AND/OR pattern mining method has the following advantage compared with the discriminative pattern mining method in [3]: our method can directly mine the optimal AND/OR pattern from the weighted transaction dataset provided by the boosting framework, while the two-stage method in [3] had ignored the sample weights when generating frequent pattern candidates in the first stage and thus cannot guarantee discovering the optimal pattern for boosting in the second stage. Therefore our proposed optimal AND/OR pattern mining method is more consistent to be combined with the boosting algorithm and is expected to perform better compared with the two-stage method in [3].

The accuracy results are shown in Table 4.3. Since the AND/OR pattern can be a combination of both LCC and CENTRIST features, it is likely to be more discriminative than the decision stumps on individual features. We find that boosting with only AND patterns has achieved a very decent performance $89.9 \%$ compared with boosting with decision stumps result $85.9 \%$ 

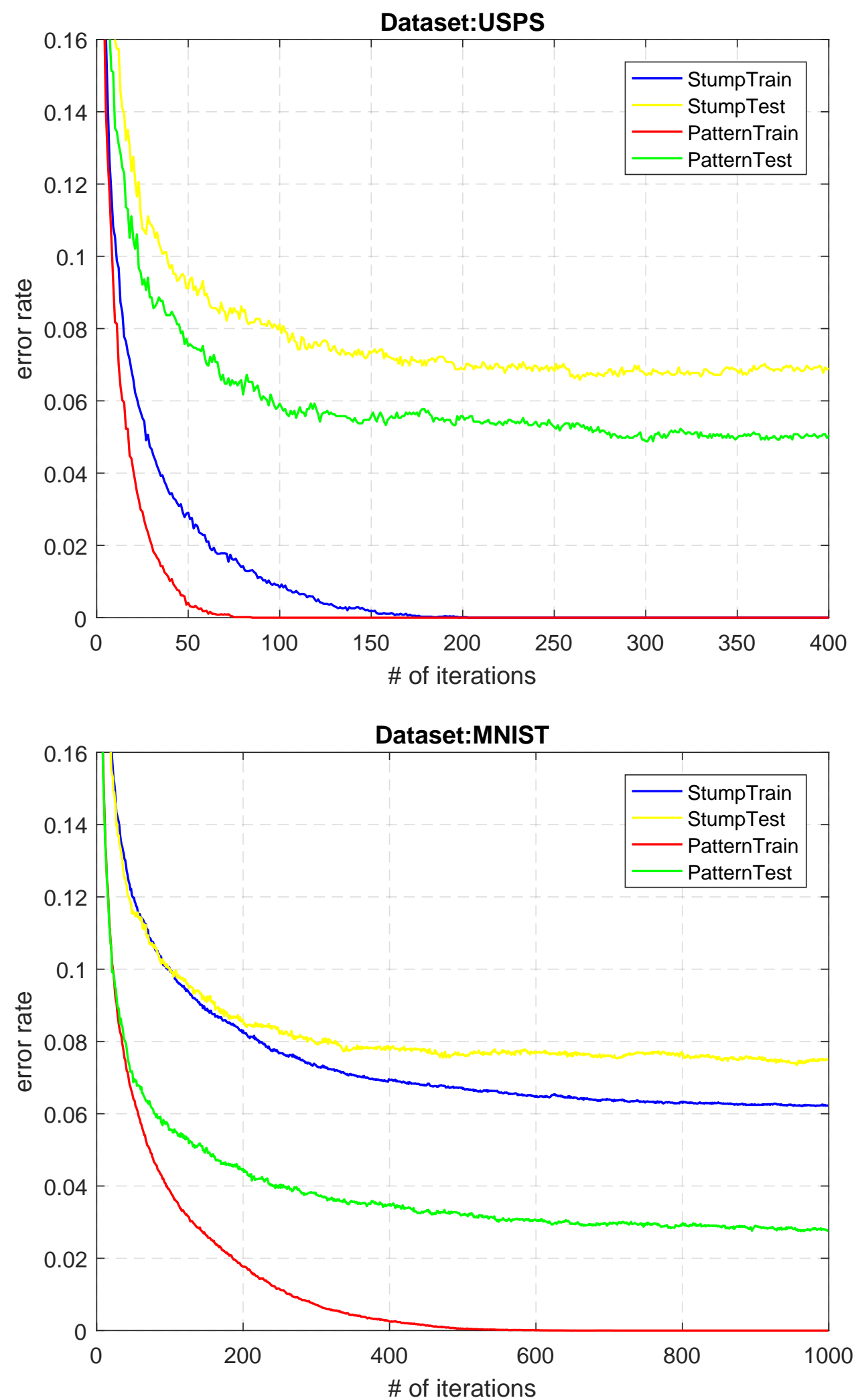

FIGURE 4.3. Learning curve comparisons between boosting with decision stumps and our boosting with AND/OR patterns. 

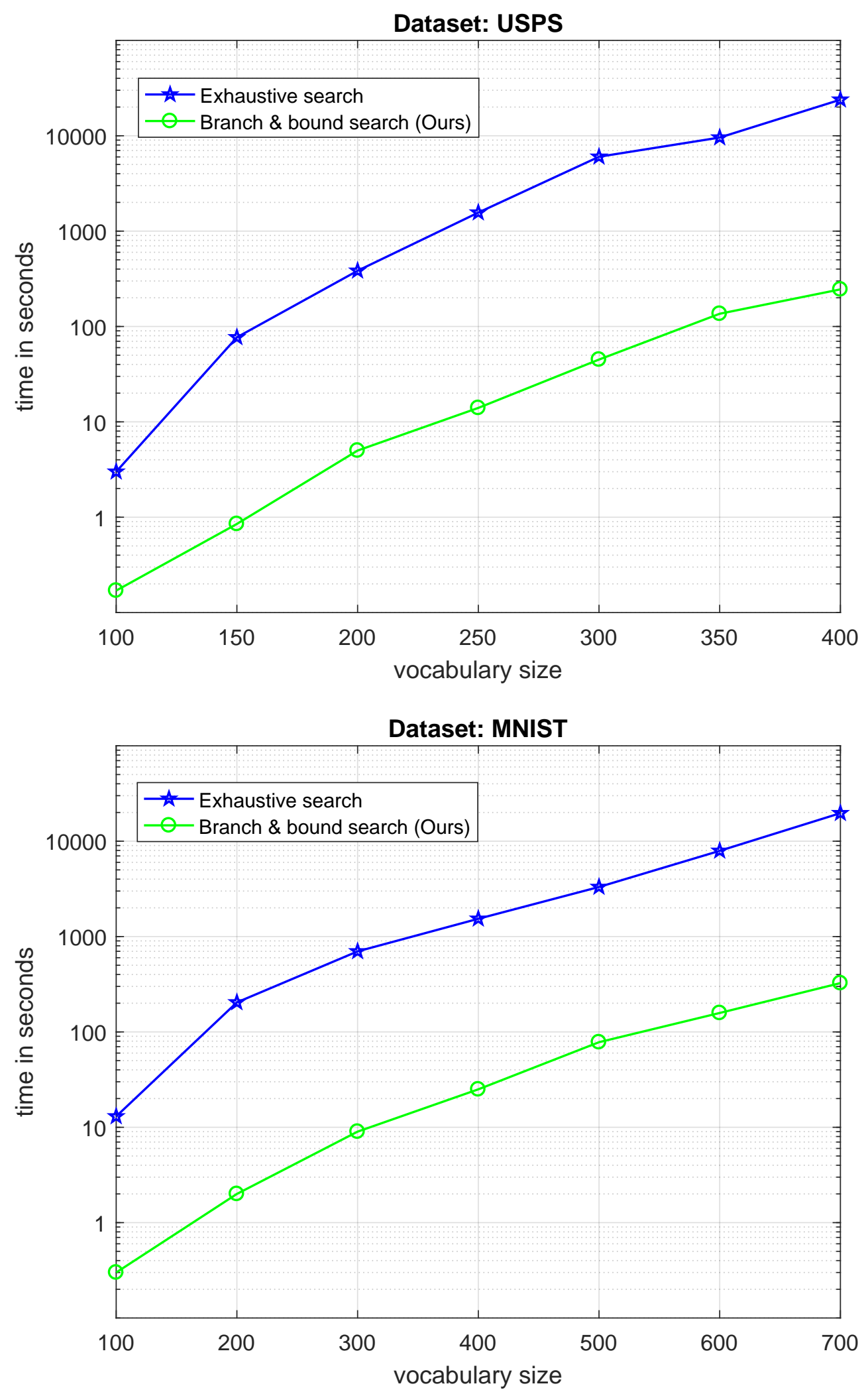

FIGURE 4.4. Running time comparisons between the two-round two-stage pattern mining method [3] and our branch-and-bound method. 
from [3]. We also find that when using both AND/OR patterns together for boosting, we can further improve the performance on the Scene-15 dataset and achieve the state-of-the-art result $91.0 \%$. It is also worth noting that our boosting with optimal AND/OR patterns method has improved the previous boosting with discriminative AND/OR patterns from $87.8 \%$ to $91.0 \%$ by $3.2 \%$. The confusion matrix of the best result achieved is shown in Fig. 4.5.

We also show the number of discovered patterns of different orders in Table 4.4. Since we have 15 class training samples and 1000 iterations for the boosting algorithm, we can obtain in total $15 \times 1000=15000$ patterns. As shown in the table, we have discovered 125391 order patterns, i.e., the decision stumps, and 2461 high-order patterns. Although the number of high-order patterns are much less than decision stumps, the final superior performance shown in Table 4.3 has demonstrated the advantage of the high-order patterns.

In summarization, thanks to the weighted transaction dataset, our proposed optimal pattern mining method can be more effective when combined with the boosting algorithm than the twostage discriminative pattern mining method in [3]. Moreover, we also discover that by exploring more potential pattern candidates, boosting with AND/OR patterns can be more discriminative than boosting with only AND patterns.

\begin{tabular}{|l|c|}
\hline Algorithm & Accuracy (\%) \\
\hline \hline SPM + SIFT with 400 clusters [22] & 81.4 \\
\hline SPM + SIFT with 400 concepts [111] & 83.3 \\
\hline SP-pLSA + SIFT with 1200 topics [112] & 83.7 \\
\hline \hline CENTRIST + SVM [74] & 83.9 \\
\hline LCC + SVM [3] & 80.7 \\
\hline CENTRIST \& LCC + Boost stump [3] & 85.9 \\
\hline CENTRIST \& LCC + Boost discriminative AND/OR [3] & 87.8 \\
\hline \hline CENTRIST \& LCC + Boost optimal AND (Ours) & 89.9 \\
\hline CENTRIST \& LCC + Boost optimal AND/OR (Ours) & $\mathbf{9 1 . 0}$ \\
\hline
\end{tabular}

TABle 4.3. Accuracy results of the Scene-15 dataset. 


\begin{tabular}{|c|c|c|c|c|c|c|c|c|c|c|c|c|c|c|}
\hline bedroom & 75.9 & 17.2 & 0 & $4.3 \quad 0.9$ & 0 & 0 & 0 & 0 & 0 & 0 & 0 & 0 & 0.9 & 0.9 \\
\hline livingroom & -7.4 & 87.8 & 0 & 0.51 .1 & 0 & 0 & 0 & 0.5 & 0 & 0 & 0 & 0 & 1.1 & 1.6 \\
\hline suburb & -0 & 0.7 & 99.3 & 0 & 0 & 0 & 0 & 0 & 0 & 0 & 0 & 0 & 0 & 0 \\
\hline industrial & -0 & 0 & 0 & 86.70 .5 & 0 & 0 & 0 & 0.9 & 0 & 0.5 & 0.5 & 3.8 & 0 & 7.1 \\
\hline kitchen & -8.2 & 4.5 & 0 & 2.778 .2 & 0 & 0 & 0 & 0 & 0 & 0 & 0 & 0.9 & 0 & 5.5 \\
\hline coast & -0 & 0 & 0 & 0.40 & 91.2 & 0.4 & 0.8 & 0 & 0 & 6.9 & 0 & 0.4 & 0 & 0 \\
\hline forest & -0 & 0 & 0 & 0 & 0 & 95.2 & 0 & 0 & 4.8 & 0 & 0 & 0 & 0 & 0 \\
\hline highway & -0.6 & 0 & 0 & 0 & 1.9 & 0 & 87.5 & 0.6 & 1.9 & 6.3 & 0.6 & 0 & 0 & 0.6 \\
\hline inside city & -0.5 & 0.5 & 0 & 0.5 & 0 & 0 & 0 & 93.3 & 0 & 0 & 3.8 & 1.0 & 0.5 & 0 \\
\hline mountain & -0 & 0 & 0 & 0 & 0.7 & 0.4 & 0 & 0 & 98.2 & 0.7 & 0 & 0 & 0 & 0 \\
\hline pen country & -0 & 0 & 0 & 0 & 4.5 & 3.2 & 0.6 & 0 & 4.5 & 87.1 & 0 & 0 & 0 & 0 \\
\hline street & -0 & 0 & 0 & 0 & 0 & 0 & 0 & 1.0 & 0 & 0 & 97.4 & 1.6 & 0 & 0 \\
\hline tall building & -0 & 0.4 & 0 & 1.20 .4 & 0 & 0 & 0 & 3.5 & 0 & 0 & 0 & 93.8 & 0 & 0.8 \\
\hline office & -1.7 & 0.9 & 0 & 4.3 & 0 & 0 & 0 & 0 & 0 & 0 & 0 & 0 & 93.0 & 0 \\
\hline store & $-1,9$ & 1,4 & 0 & $2,3 \quad 2,3$ & Q & 0,5 & Q & 0,9 & 0 & 오 & Q & 0,9 & 0,5 & 89.3 \\
\hline
\end{tabular}

FIGURE 4.5. The confusion matrix of the Scene-15 dataset.

\begin{tabular}{|l|c|c|c|c|c|c|c|c|c|}
\hline Order & $\mathbf{1}$ & $\mathbf{2}$ & $\mathbf{3}$ & $\mathbf{4}$ & $\mathbf{5}$ & $\mathbf{6}$ & $\mathbf{7}$ & $\mathbf{8}$ & $\mathbf{9}$ \\
\hline Number & 12539 & 483 & 762 & 750 & 320 & 109 & 30 & 5 & 2 \\
\hline
\end{tabular}

TABLE 4.4. The number of discovered patterns of different orders on the Scene- 15 dataset.

\subsubsection{Action Recognition Dataset}

In this section we also apply our proposed boosting with optimal AND/OR patterns method on the MSR Daily Activity 3D [10] dataset for action recognition problem.

MSR Daily Activity 3D [10] dataset is a daily activity dataset that includes the RGB frames, depth sequences and the tracked skeleton joint positions from Microsoft's Kinect camera. It contains in total 16 daily activities from 10 subjects, i.e., drink, eat, read book, call cellphone, write on a paper, use laptop, use vacuum cleaner, cheer up, sit still, toss paper, play game, lay down on sofa, walk, play guitar, stand up, sit down. Each subject performs an activity in two different poses: sitting on sofa and standing. The dataset contains totally 320 daily activity sequences. 


\begin{tabular}{|l|c|c|c|c|c|c|c|c|}
\hline Order & $\mathbf{1}$ & $\mathbf{2}$ & $\mathbf{3}$ & $\mathbf{4}$ & $\mathbf{5}$ & $\mathbf{6}$ & $\mathbf{7}$ & $\mathbf{8}$ \\
\hline Number & 11262 & 1047 & 1334 & 1011 & 758 & 522 & 60 & 6 \\
\hline
\end{tabular}

TABLE 4.5. The number of discovered patterns of different orders on the MSR Daily Activity $3 \mathrm{D}$ dataset.

In the experiment, we extract the action features provided by the actionlet ensemble work [10]. That is, at each frame we first extract the 3D joint relative position feature and the Local Occupancy Pattern (LOP) feature, which can not only represent the human motions but also the interactions between the objects and the human. After that, we use the Fourier Temporal Pyramid (FTP) to capture the temporal dynamics of the previously extracted frame-level features. The final extracted FTP features are translation-invariant to the human bodies and also robust to temporal misalignment and data noise. We conduct our experiments on the cross-subject test setting, i.e., samples of the subjects $1,2,3,4,5$ are used as training data and samples of the subjects $6,7,8,9,10$ are used as testing data, as in [10].

To validate the advantages of our optimal AND/OR pattern mining algorithm, we employ the same experimental setup as [10], except for the discriminative actionlet mining step. In the experiment, we run boosting algorithm for 1000 iterations to select the AND/OR patterns. The final recognition accuracy results are shown in Table 4.6. From the table we can see that, when only the $3 \mathrm{D}$ joint relative position features are employed, our pattern mining method improves the recognition accuracy from $74 \%$ to $76.25 \%$ compared with the pattern mining method used in [10]. If we only use the Local Occupancy Pattern (LOP) features as base features, we also observe a performance improvement from $61 \%$ to $62.5 \%$. We also find that on the Fourier Temporal Pyramid (FTP) features that are more robust to data noise and temporal misalignment [10], our optimal AND/OR pattern mining algorithm achieves the best recognition accuracy $86.88 \%$, which has superior performance than the state-of-the-art result $85.75 \%$ in [10]. The confusion matrix of the best result achieved is shown in Fig. 4.6. We also show the number of discovered patterns of different orders in Table 4.5.

In summarization, compared with the pattern mining method in the actionlet work [10] that heuristically discovered discriminative AND patterns for ensemble, our proposed method can 
simultaneously discover the optimal AND and OR patterns to be integrated in boosting algorithm. Thanks to the discovered optimal AND/OR patterns, our proposed method achieves superior action recognition performance on the MSR Daily Activity 3D dataset compared with the previous actionlet work [10].

\begin{tabular}{|l|c|}
\hline Algorithm & Accuracy (\%) \\
\hline \hline Dynamic temporal warping [113] & 54.0 \\
\hline Random occupancy pattern [43] & 64.0 \\
\hline \hline LOP features + SVM [10] & 43.0 \\
\hline joint features + SVM [10] & 68.0 \\
\hline FTP features + SVM [10] & 78.0 \\
\hline \hline LOP features + Actionlet ensemble [10] & 61.0 \\
\hline joint features + Actionlet ensemble [10] & 74.0 \\
\hline FTP features + Actionlet ensemble [10] & 85.75 \\
\hline \hline LOP features + Boost optimal AND/OR patterns (Ours) & 62.5 \\
\hline joint features + Boost optimal AND/OR patterns (Ours) & 76.25 \\
\hline FTP features + Boost optimal AND/OR patterns (Ours) & $\mathbf{8 6 . 8 8}$ \\
\hline
\end{tabular}

TABLE 4.6. Accuracy results of the MSR Daily Activity 3D dataset.

\subsection{Conclusion and Future Work}

We propose a novel branch-and-bound search based approach to efficiently mine the optimal AND/OR co-occurrence features with minimal weighted training error for boosting. Experimental results on benchmark datasets show that boosting with co-occurrence features provides very competitive results compared with other types of base classifiers such as decision stumps and decision trees. The proposed optimal AND/OR co-occurrence feature mining algorithm also achieves orders of magnitude speed up than previous two-round two-stage discriminative pattern mining algorithm. Besides UCI datasets, our method also achieves the state-of-the-art results on the Scene-15 recognition dataset and the MSR Daily Activity 3D dataset.

There are still many potential extensions to this work. For example, it will be interesting if we can apply the proposed method to discover AND/OR patterns in convolution neural networks to improve visual recognition performance. The AND/OR patterns can be composed by feature 


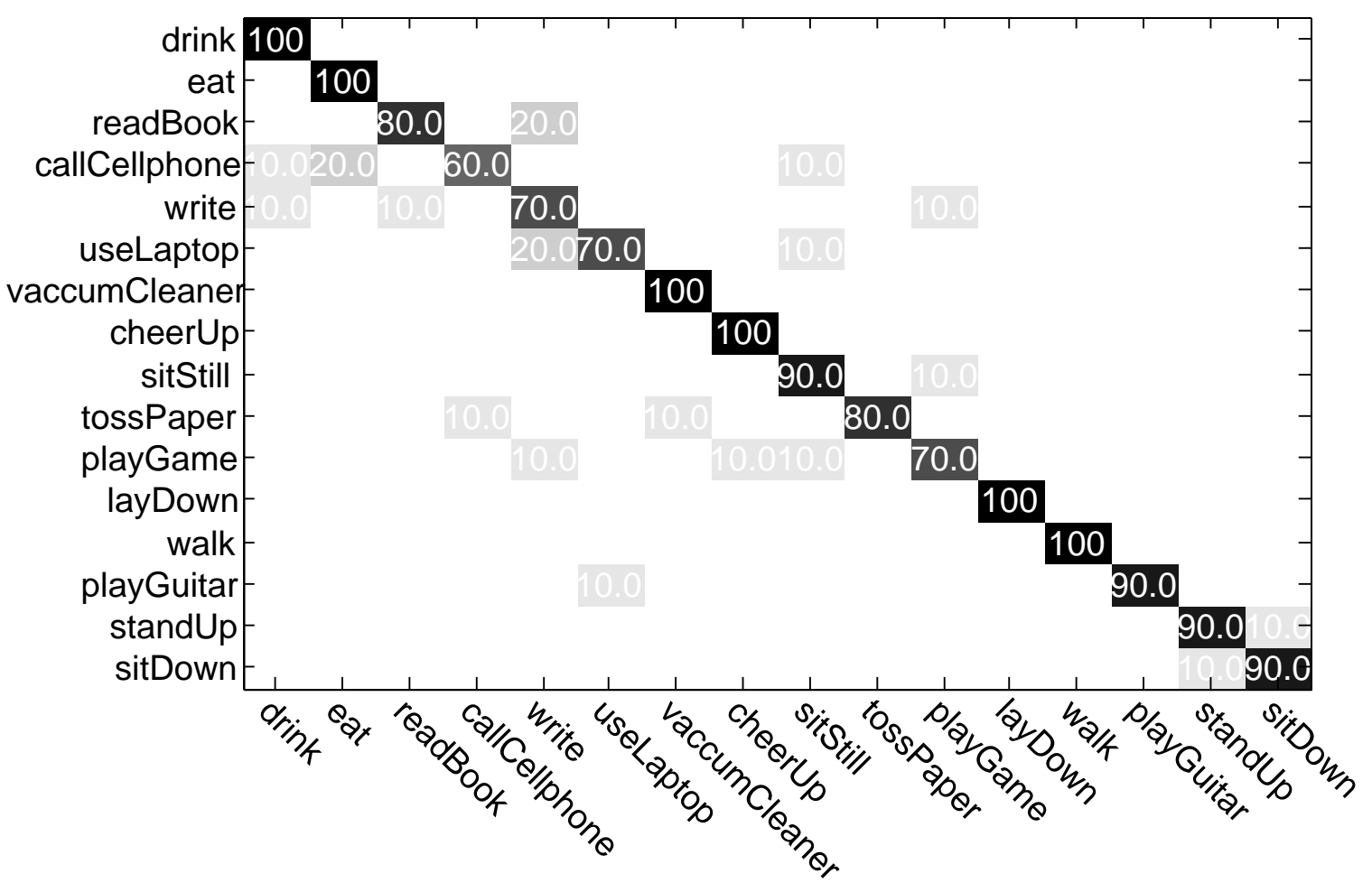

FIGURE 4.6. The confusion matrix of MSR Daily Activity 3D dataset.

map responses from the same neural network layer, and also from different neural network layers to take advantage of multi-scale feature maps. 


\section{Chapter 5}

\section{Learning Attentional AND/OR Skeleton}

\section{Patterns for 3D Action Recognition}

\subsection{Introduction}

Human action recognition has been one of the most important applications in computer vision. In the recent years, due to the development of depth sensors such as Microsoft Kinect and the successes of human body modeling algorithms, skeleton based action recognition has become more and more attractive [10, 114-116].

Although depth cameras can provide estimated human skeleton joints from 3D depth image data, it is still challenging to extract discriminative feature information from skeleton joints and raw depth image for human action recognition. Single individual 3D skeleton joint feature cannot be discriminative enough to distinguish different human actions since human skeleton joints are usually spatially correlated, as can be seen from Fig. 5.1. For example, for the action "high arm waving" shown in Fig. 5.1, left hand, left wrist, left elbow, left shoulder often move simultaneously in groups. Therefore ignoring the spatial co-occurrence of skeleton joints can result in inferior action recognition performance. 
However, how to efficiently discover high order discriminative co-occurrence patterns features still remains a challenging problem. The first challenge comes from the huge number of possibilities of composing high-order co-occurrence features. Given $N$ binary features, in total we can obtain a pool of $2^{N}$ (or $3^{N}$ if considering both positive and negative responses) cooccurrence features [3], from which it is a computational demanding task to seek the discriminative patterns. In practical tasks, the value of $N$ could be hundreds or even larger, and it is computationally intractable to perform an exhaustive search in the exponentially large number of candidates. To reduce the exhaustive search time, some existing approaches propose to search for fixed-order co-occurrence features [28, 81], or use the sequential forward selection algorithm $[89,96]$. However, these approaches cannot guarantee the optimality of the discovered co-occurrence features, hence resulting in less effective features. The actionlet work [10] has proposed a heuristic method to exhaustively search for high order skeleton patterns with high confidence and ambiguity scores, however it cannot guarantee the optimality of the discovered patterns and also cannot be affordable when the feature pool is of large size.

Besides, after the discriminative co-occurrence features are discovered, how to integrate them appropriately in spatial and temporal domain for human action recognition also needs to be studied. Due to the large intra-class variations of action categories, one single co-occurrence pattern is usually not enough to distinguish different action categories. In the actionlet work [10], the discovered skeleton patterns are combined in an ensemble via multiple kernel learning. Many recent attempts also propose to use Recurrent Neural Network (RNN) based temporal models, such as $[115,115-117]$. However, it is rather difficult to interpret internal representations learned by these works. Attention model $[86,118]$ can be used to capture where the model is focusing and make it more human-interpretable for human action recognition application.

In this chapter, we aim at efficiently mining discriminative high order AND/OR skeleton patterns from raw skeleton features in the spatial domain and also learning visual attentions of the mined skeleton patterns in the temporal domain, to exploit both spatial and temporal context information for not only boosting action recognition performance but also capturing the essentials of actions in the human-interpretable way by detecting visual attentions. In the spatial domain, 


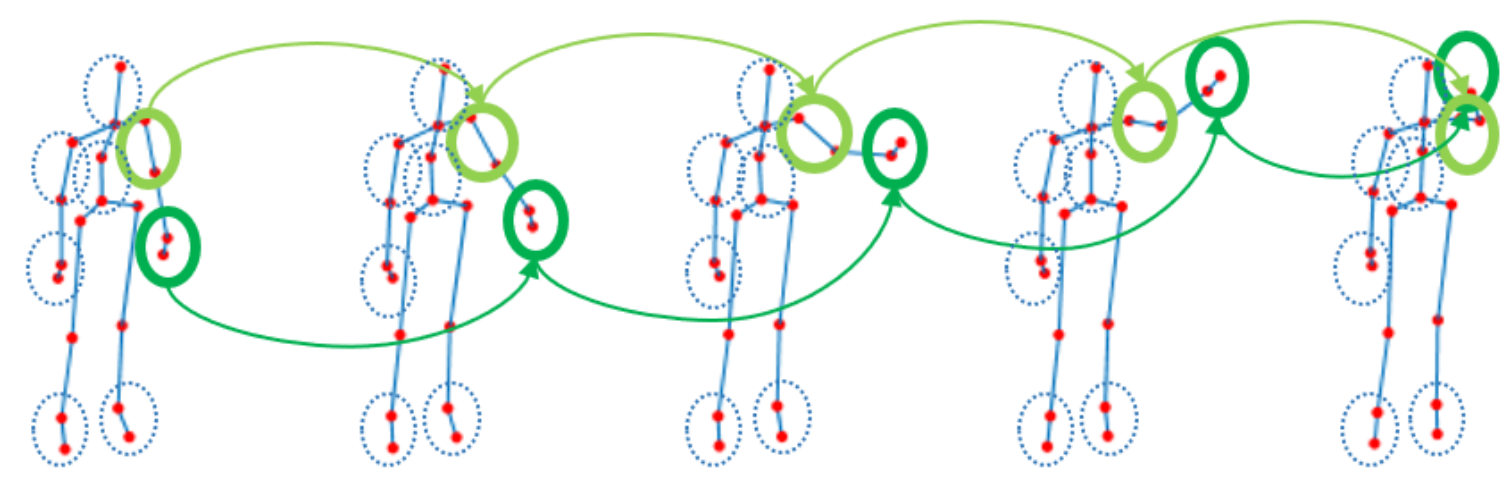

$\Longrightarrow$ Time

FIGURE 5.1. Illustration of high order patterns of skeleton joints in spatial domain and moving visual attentions in temporal domain for action category "high arm waving". In the figure, we can see two spatial co-occurrence patterns, one formed by left shoulder and left elbow showed in light blue circle, the other containing left wrist and left hand showed in deep blue circle, moving in time dimension. Since the other skeleton joints are almost stationary, it is clear that visual attentions in temporal dimension will follow the movements of the two spatial patterns for action category "high arm waving".

we propose to mine discriminative high order AND/OR patterns of skeleton joints from labeled multi-class dataset to incorporate the spatial context information of skeleton joints. To speed up the data mining process, we propose an efficient branch and bound search algorithm and significantly boost the pattern mining process by orders of magnitude. In the temporal domain, we propose to model visual attentions over time by selectively focusing on specific parts of the discovered skeletons that are relevant for the action recognition task at hand. By doing so, we can not only boost the action recognition performance, but also have better visualizations of what have been learned. Experiments on MSR Daily Activity, MSR 3D Action, SBU interaction and NTU-RGBD dataset for 3D action recognition show both the effectiveness and efficiency of our proposed methods.

The remainder of this chapter is organized as follows: In Sec. 5.2 we have a literature review. In Sec. 5.3 we introduce our proposed high order AND/OR pattern mining algorithm and the attention LSTM model. In Sec. 5.4 we conduct our experiments. In Sec.5.4.5 we have a conclusion. 


\subsection{Related Work}

Human action recognition using 3D skeleton joint features [10, 114-116, 119] has been popular in the recent years due to the development of depth sensors such as Microsoft Kinect[120].

In the literature, there have been many approaches to discover skeleton patterns for skeleton based action recognition. The random occupancy pattern (ROP) work [114] randomly sampled subvolumes in the $4 \mathrm{D}$ space $(\mathrm{x}, \mathrm{y}, \mathrm{z}, \mathrm{t})$ and then computed the histogram of cloud points that fall into the subvolume as the skeleton feature representation. After that, it also proposed to select the discriminative ROPs via feature selection algorithm and then trained an SVM classifier for action recognition. The bag of frequent local parts work in [121] employed frequent pattern mining algorithm to mine the most frequent and relevant (discriminative, representative and non-redundant) states of skeleton parts and then formed the bag of feature representation for action recognition. The actionlet ensemble work [10] introduced pairwise relative joint position feature, local occupancy pattern feature and Fourier temporal pyramid feature for skeleton feature representations. In addition to the skeleton features, they also proposed to mine conjunction patterns of skeleton features using a heuristic approach similar to Apriori algorithm [12]. After mining the patterns, they applied multiple kernel learning for action classification.

Unlike the above mentioned pattern discovery methods that used heuristic approaches to discover frequent or relevant patterns and thus cannot guarantee the optimal solution for classification, our proposed pattern mining algorithm can guarantee to directly mine the optimal AND/OR pattern with maximum information gain from multi-class dataset. What's more, previous methods often suffered from the time-consuming process when mining the frequent patterns, while on the contrary, our proposed pattern mining algorithm employs the branch and bound search mechanism and can be orders of magnitude faster than exhaustive search methods such as $[10,121]$.

There are also AND/OR graph (AOG) mining works in the literature of computer vision, however they are mainly applied in image/video data analysis. For example, [122] proposed an 
AND-OR grammar model based on image contents to parse human attributes, parts and poses. The work in [123] proposed to gradually grow a semantically graphical model on the pretrained CNN for the hierarchical understanding of images. The work in [124] proposed to mine maximal-size common subgraphs from a set of attributed relational graphs on images/videos. It is worth noting that our proposed AND/OR pattern mining algorithm mainly focuses on extracting discriminative and human-interpretable mid-level skeleton patterns to boost action recognition performance, while theirs focused more on parsing objects in the images/videos via AND-OR graph models.

There are many approaches in the literature of temporal modeling for skeleton based action recognition. The HBRNN work [115] constructed bidirectional RNNs upon 3D skeleton joints in a hierarchical fashion for action recognition. It pre-defined 5 groups for skeleton joints and trained separated RNNs. After that it concatenated the outputs of these BRNNs to form a new layer. This process is repeated for several times and finally a fully connected layer and a softmax layer are performed on the final representation to classify the actions. The cooccurrence LSTM work [119] applied deep LSTM model with co-occurrence regularization on the network weights to discover discriminative skeleton joints for action recognition. It also introduced an in-depth dropout technique to further improve the performance of the deep LSTM model. The ST-LSTM algorithm [116] proposed a tree structure based skeleton traversal method to explore the context relationship between skeleton joints in both spatial domain and temporal domain.

Different from these LSTM models that only aimed at improving the classification performance, our proposed attention LSTM model can selectively focus on specific parts of skeleton patterns that are relevant for action recognition task. By identifying the specific skeleton patterns that draw the visual attentions, our proposed model can capture the essentials of actions and thus help boost recognition performance in a human-interpretable way. 


\subsection{Proposed Method}

\subsubsection{Skeleton Features}

For a human action, 20 skeleton joints can be tracked using a real time skeleton tracker [120]. As observed in [10], relative skeleton positions can be more discriminative than absolute skeleton positions. Assume each skeleton is represented by $p_{i} \in R^{3}$, we compute the pairwise relative joint positions $p_{i, j}$ as the feature representation $\mathbf{r}_{i}$ for each skeleton joint $p_{i}$, as follows:

$$
\begin{array}{r}
p_{i, j}=p_{i}-p_{j} \\
\mathbf{r}_{i}=\left\{p_{i j} \mid i \neq j\right\}
\end{array}
$$

The relative joint position feature can represent human poses and motions, however it still lacks the context information. For example, for action category "playing guitar", local regions around hand and elbow skeleton joints will be filled with cloud points of guitar in depth image. These local cloud points plays an import role in distinguish "playing guitar" action from other actions such as "drink" and "eat". Therefore we also compute the local occupancy pattern (LOP) feature [10] around each skeleton joint to obtain the depth appearance of each skeleton joint.

For each skeleton joint $p_{i}$, its local region in the 3D cloud point space is partitioned into $N_{x} \times$ $N_{y} \times N_{z}$ spatial grids. The LOP feature is computed by counting the number of cloud points that fall into each spatial grid $n_{x, y, z}$ and forming a histogram of all the spatial grids $\mathbf{s}_{i}$, as follows:

$$
\begin{aligned}
n_{x, y, z} & =\sigma\left(\sum_{q \in b i n_{x, y, z}} I(q)\right) \\
\mathbf{s}_{i} & =\left\{n_{x, y, z} \mid \forall x, y, z\right\}
\end{aligned}
$$

where $I(q)$ is an indicator function determining whether a point $q$ is inside the spatial grid $\operatorname{bin}_{x, y, z}$, and $\sigma(\cdot)$ is the sigmoid function used for normalization. 
After extracting relative joint position feature $\mathbf{r}_{i}$, and LOP feature $\mathbf{s}_{i}$, we combine them to form the skeleton feature representation $\mathbf{x}_{i}$ for each skeleton joint $p_{i}$, as follows:

$$
\mathbf{x}_{i}=\left[\mathbf{r}_{i}^{T} ; \mathbf{s}_{i}^{T}\right]^{T}
$$

\subsubsection{AND/OR Pattern Mining}

It is clear that skeleton joints in the spatial domain are highly correlated and often move as groups in the temporal domain. For example, as shown in Fig. 4.1, left shoulder, left elbow, left wrist, left hand are shown to be more discriminative than other skeleton joints for action category "high arm waving". Therefore it is worth mining high order patterns of skeleton joints for action recognition.

How to mine discriminative patterns from multi-class labeled dataset is still a challenging problem. We propose to discover discriminative patterns via maximizing information gain of multiclass labeled dataset. Due to the huge amount of candidate high order patterns (up to $2^{N}$ conjunction candidates given $N$ raw binary features and $3^{N}$ conjunction/disjunction candidates), data mining algorithms are known to be very time-consuming. To speed up the pattern mining process, we derive an upper bound of the information gain criteria with respect to pattern frequency and propose an efficient branch and bound search method based on the derived upper bound.

In the following we will first introduce the pattern mining pipeline using the extracted skeleton feature from Sec. 5.3.1. After that, we will introduce our proposed branch and bound search approach to speed up the pattern mining process.

\subsubsection{Pattern Mining Overview}

We show our proposed AND/OR pattern mining overview in Fig. 5.2, which is similar to Sec. 4.3 in Chapter 4. It is worth noting that the criterion to mine the optimal AND/OR pattern in Chapter 4 is classification error, while in this chapter it is information gain. 
In the following we will briefly introduce the overview of mining optimal pattern with maximum information gain from labeled transaction dataset. An example dataset with labeled and weighted data samples is shown in step (a) of Fig. 5.2. Given the multi-class real-valued dataset, we first apply feature binarization to obtain the transaction dataset that represents data samples using discrete items, as shown in step (b) and (c) of Fig. 5.2. For feature binarization, we exhaustively search all the thresholds of each feature dimension and find the optimal one with maximal information gain.

After generating the transaction dataset, we apply an efficient branch and bound search method to discover the optimal pattern with maximal information gain in conjunction (AND) form or disjunction (OR) form, which will be further discussed in Sec. 5.3.2.4. According to De Morgan's Law, conjunction pattern and disjunction pattern can convert to each other. Our datadriven pattern mining algorithm will determine the form of the discovered optimal pattern from the labeled dataset directly. As shown in step (d) of Fig. 5.2, we discover a disjunction pattern from the example dataset.

Considering that single AND/OR pattern may not be discriminative enough for classification task, we mine multiple patterns recursively to form a decision tree, as shown in step (e) of Fig. 5.2. In order to reduce variance [125], we also apply bagging method to generate multiple decision trees.

Finally, after we generate multiple trees using discovered high order patterns, we can input each data sample $\mathbf{x}_{i}$ into the trees and obtain the histogram representations in the leaves as feature vectors $\mathrm{x}_{i}^{\prime}$ for the discovered high order AND/OR patterns, as follows:

$$
\mathbf{x}_{i}^{\prime}=\left\{\operatorname{hist}_{j}\left(\mathbf{x}_{i}\right) \mid \forall j\right\}
$$

where $\operatorname{hist}_{j}(\cdot)$ is the histogram in the leave of the $j$-th tree.

In the following we will discuss how to efficiently mine the optimal AND/OR pattern with maximal information gain from multi-class transaction dataset. 


\begin{tabular}{|c|c|c|c|c|c|c|}
\hline$y_{i}$ & $w_{i}$ & $x_{i}$ & \multirow{4}{*}{$\Rightarrow$} & $x_{i}$ & \multirow{4}{*}{$\Longrightarrow$} & $x_{i}$ \\
\hline 1 & 0.4 & $\{0.5,0.1 \ldots 0.2\}$ & & $\{1,0 \ldots 0\}$ & & $\left\{e_{1}, \overline{e_{2}} \ldots \overline{e_{T}}\right\}$ \\
\hline 1 & 0.2 & $\{0.7,0.2 \ldots 0.3\}$ & & $\{1,0 \ldots 1\}$ & & $\left\{e_{1}, \overline{e_{2}} \ldots e_{T}\right\}$ \\
\hline 2 & 0.3 & $\{0.6,0.4 \ldots 0.4\}$ & & $\{1,1 \ldots 1\}$ & & $\left\{e_{1}, e_{2} \ldots e_{T}\right\}$ \\
\hline 2 & 0.1 & $\{0.4,0.3 \ldots 0.1\}$ & & $\{0,0 \ldots 0\}$ & & $\left\{\overline{e_{1}}, \overline{e_{2}} \ldots \overline{e_{\mathrm{T}}}\right\}$ \\
\hline
\end{tabular}

(a) Multi-class read-valued dataset

(b) Feature binarization

(c) Transaction dataset

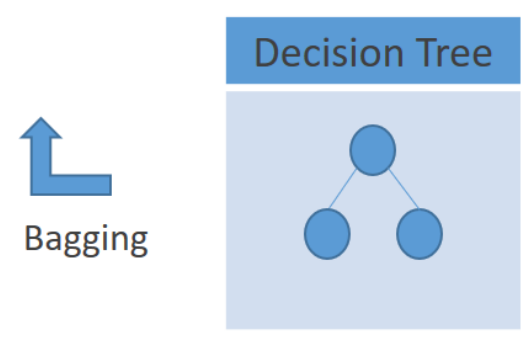

(e) Decision Tree

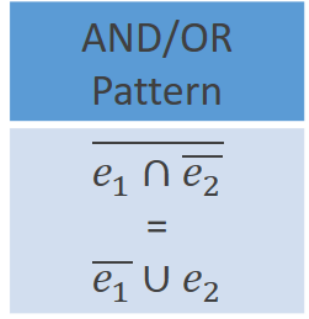

(d) AND/OR Pattern

FIGURE 5.2. Illustration of the AND/OR pattern mining pipeline.

\subsubsection{Information Gain for Pattern Mining}

Before introducing the proposed branch and bound search method for efficient pattern mining from multi-class transaction dataset, we first derive the upper bound of information gain with respect to pattern frequency.

Formally, assume we are given a pattern represented by a random variable $X \in\{0,1\}$ and dataset label is represented by $Y=\{1,2, \cdots K\}$, the information gain of the dataset can be computed as follows:

$$
I G(Y \mid X)=H(Y)-H(Y \mid X)
$$

where $H(Y)$ is the entropy and $H(Y \mid X)$ is the conditional entropy. Since $H(Y)$ is a constant given the dataset, in the following we only consider $-H(Y \mid X)$. 
We first define $\theta_{i}$ as the joint probability and $c_{i}$ as the marginal probability as follows:

$$
\begin{array}{r}
\theta_{i}=P(y=i, x=0) \\
c_{i}=P(y=i)
\end{array}
$$

We can compute the following probabilities based on $\theta_{i}$ and $c_{i}$ :

$$
\begin{aligned}
P(y=i, x=1) & =c_{i}-\theta_{i} \\
P(x=0) & =\sum_{i=1}^{K} \theta_{i} \\
P(x=1) & =1-\sum_{i=1}^{K} \theta_{i} \\
P(y=i \mid x=0) & =\theta_{i} / \sum_{i=1}^{K} \theta_{i} \\
P(y=i \mid x=1) & =\left(c_{i}-\theta_{i}\right) /\left(1-\sum_{i=1}^{K} \theta_{i}\right)
\end{aligned}
$$

We can then rewrite the function $-H(Y \mid X)$ as follows:

$$
\begin{aligned}
& -H(Y \mid X) \\
= & \sum_{x \in\{0,1\}} P(x) \sum_{y \in\{1, \cdots, K\}} P(y \mid x) \log P(y \mid x) \\
= & \sum_{j=0}^{1} P(x=j) \sum_{i=1}^{K} P(y=i \mid x=j) \log (P(y=i \mid x=j)) \\
= & \sum_{i=1}^{K} \theta_{i} \log \left(\frac{\theta_{i}}{\sum_{i=1}^{K} \theta_{i}}\right)+\sum_{i=1}^{K}\left(c_{i}-\theta_{i}\right) \log \left(\frac{c_{i}-\theta_{i}}{\sum_{i=1}^{K}\left(c_{i}-\theta_{i}\right)}\right) \\
= & \sum_{i=1}^{K} \theta_{i} \log \left(\theta_{i}\right)+\sum_{i=1}^{K}\left(c_{i}-\theta_{i}\right) \log \left(c_{i}-\theta_{i}\right) \\
= & \sum_{i=1}^{K} v\left(\theta_{i}, c_{i}\right)-v\left(\sum_{i=1}^{K} \theta_{i}, \sum_{i=1}^{K} c_{i}\right)
\end{aligned}
$$


where each $v\left(\theta_{i}, c_{i}\right)$ is defined as:

$$
v\left(\theta_{i}, c_{i}\right)=\theta_{i} \log \left(\theta_{i}\right)+\left(c_{i}-\theta_{i}\right) \log \left(c_{i}-\theta_{i}\right)
$$

As shown in Eq. 5.9, each $c_{i}$ is a constant given the dataset. Therefore from Eq. 5.16 we can see that $-H(Y \mid X)$ is a function of pattern frequencies in different classes $\left\{\theta_{i} \mid \forall i \in\{1,2, \cdots, K\}\right\}$.

\subsubsection{Information Gain Upper Bound}

We can compute the first and second derivatives $\frac{\partial v}{\partial \theta_{i}}$ and $\frac{\partial^{2} v}{\partial \theta_{i}^{2}}$ of $v\left(\theta_{i}, c_{i}\right)$ as follows:

$$
\begin{aligned}
\frac{\partial v}{\partial \theta_{i}} & =\log \left(\frac{\theta_{i}}{c_{i}-\theta_{i}}\right) \\
\frac{\partial^{2} v}{\partial \theta_{i}^{2}} & =\frac{1}{\ln 2}\left(\frac{1}{\theta_{i}}+\frac{1}{c_{i}-\theta_{i}}\right)
\end{aligned}
$$

From the definitions of pattern frequency $\theta_{i}$ and class weight $c_{i}$, we know that $\theta_{i}$ and $c_{i}$ subject to the constraints as follows:

$$
\begin{array}{r}
0 \leq \theta_{i} \leq c_{i} \leq 1 \\
\sum_{i} c_{i}=1
\end{array}
$$

From the constraint in 5.20, we know that $\frac{\partial^{2} v}{\partial \theta_{i}^{2}} \geq 0$ and therefore $v\left(\theta_{i}, c_{i}\right)$ is a convex function with respect to $\theta_{i}$. Due to the symmetry in function $v\left(\theta_{i}, c_{i}\right)$, we know that when $\theta_{i}$ reaches 0 or $c_{i}$, function $v\left(\theta_{i}, c_{i}\right)$ reaches its maxima $v\left(0, c_{i}\right)$, i.e.,

$$
\left.v\left(\theta_{i}, c_{i}\right)\right|_{\max }=v\left(0, c_{i}\right)
$$

By setting the first derivative $\frac{\partial v}{\partial \theta_{i}}$ to zero, we can obtain $\theta_{i}=0.5$. Since function $v\left(\theta_{i}, c_{i}\right)$ is a convex function with respect to $\theta_{i}$, we know that when $\theta_{i}$ reaches 0.5 , function $v\left(\theta_{i}, c_{i}\right)$ reaches 
its minima $v\left(0.5, c_{i}\right)$, as follows:

$$
\left.v\left(\theta_{i}, c_{i}\right)\right|_{\min }=v\left(0.5, c_{i}\right)
$$

Therefore the upper bound for information gain of a pattern $-\left.H(Y \mid X)\right|_{u b}$ can be represented as follows:

$$
\begin{aligned}
-H(Y \mid X) & <-\left.H(Y \mid X)\right|_{u b} \\
& =\sum_{i=1}^{K} v\left(0, c_{i}\right)-v\left(0.5, \sum_{i=1}^{K} c_{i}\right)
\end{aligned}
$$

\subsubsection{Branch and Bound Search}

Using the upper bound in Eq. 5.25, we propose a branch and bound search method to mine the optimal pattern using the frequent itemset mining fp-growth algorithm [13]. The basic idea is, while recursively processing the fp-tree, we can use global variables to keep track of the current best pattern and its information gain. Before processing the next conditional fp-tree, we can test the information gain upper bound of the root of the conditional fp-tree. If the upper bound is smaller than current best information gain, then we can safely skip the whole conditional fp-tree since there will not be any patterns generated from this conditional fp-tree that can have better information gain than the current best one. Due to space limit, we refer the readers to [13] for details of fp-growth algorithm.

\subsubsection{Attention Model for Skeleton Joints and Skeleton Patterns}

After obtaining high order skeleton patterns in the spatial domain, a natural question arises: how to fuse these mined skeleton patterns in the temporal domain for action recognition? As can be seen from Fig. 4.1, human actions can be characterized by the motion of skeleton joints or high order patterns of skeleton joints in the temporal domain. When comparing the movements of different skeleton joints or skeleton patterns, we can see some joints or patterns that 
draw visual attentions of human eyes contribute more than the other ones to the action label. Therefore it is an intuitive idea to model the motions of skeleton joints or skeleton patterns in temporal domain while at the same time keep tracks of the visual attentions for action recognition. In this section, we propose an LSTM model with soft attention to select discriminative skeleton joints or high order skeleton patterns over time for action recognition.

\subsubsection{Temporal Modeling with LSTM}

A typical LSTM node consists of an input gate $\mathbf{i}_{t}$, a forget gate $\mathbf{f}_{t}$, an output gate $\mathbf{o}_{t}$, an output state $\mathbf{h}_{t}$, and an internal cell state $\mathbf{c}_{t}[86,118,126]$. The transition equations of an LSTM node are shown as follows:

$$
\begin{aligned}
\left(\begin{array}{c}
\mathbf{i}_{t} \\
\mathbf{f}_{t} \\
\mathbf{o}_{t} \\
\mathbf{g}_{t}
\end{array}\right) & =\left(\begin{array}{c}
\sigma \\
\sigma \\
\sigma \\
\tanh
\end{array}\right) M\left(\begin{array}{c}
\mathbf{h}_{t-1} \\
\tilde{\mathbf{x}}_{t}
\end{array}\right) \\
\mathbf{c}_{t} & =\mathbf{f}_{t} \odot \mathbf{c}_{t-1}+i_{t} \odot \mathbf{g}_{t} \\
\mathbf{h}_{t} & =\mathbf{o}_{t} \odot \tanh \left(\mathbf{c}_{t}\right)
\end{aligned}
$$

where $\odot$ means element-wise production, $\sigma(\cdot)$ is the sigmoid activation and $\tilde{\mathbf{x}}_{t}$ is the input feature vector for the $t$-th frame and $\mathbf{g}_{t}$ is the modulated input. $M: R^{D+d} \rightarrow R^{4 d}$ is an affine transformation consisting of trainable model parameters, where $D$ is the number of dimensions of input feature vector $\tilde{\mathbf{x}}_{t}$ and $d$ is the number of LSTM cell state nodes. In the LSTM node, the input gate $\mathbf{i}_{t}$ determines how the modulated input $\mathbf{g}_{t}$ will update the internal cell state $\mathbf{c}_{t}$ at time step $t$. The forget gate will control how the previous memory cell state $\mathbf{c}_{t-1}$ will affect the current state $\mathbf{c}_{t}$. The output gate $\mathbf{o}_{t}$ determines how the internal memory cell state $\mathbf{c}_{t}$ will influence the outside nodes. 


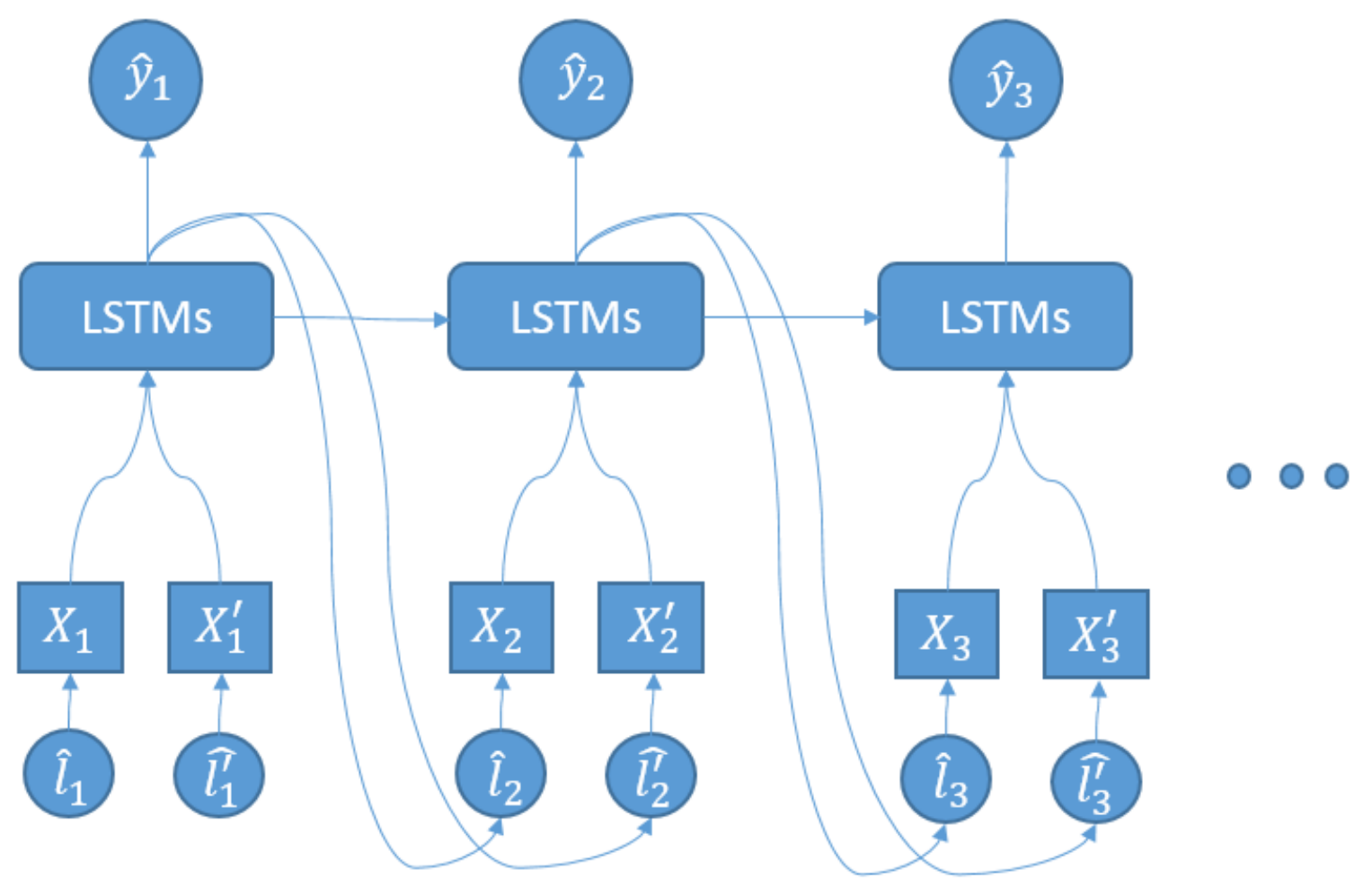

FIGURE 5.3. Illustration of attention LSTM model with joint features and high order AND/OR pattern features. In the figure, $X_{i}$ and $X^{\prime}{ }_{i}$ are the skeleton joint and skeleton pattern features for the $i^{\text {th }}$ frame respectively, $\hat{y}_{i}$ is the predicted action label, $\ell_{i}$ and $\ell_{i}^{\prime}$ are the predicted attention of skeleton joint and skeleton pattern respectively.

\subsubsection{Attention LSTM}

Formally, assume $K$ is the number of action classes, $N$ is the number of skeleton points, $N^{\prime}$ is the number of high order patterns, then at each time step $t$ we are given the skeleton joint features $X_{t}=\left\{\mathbf{x}_{t, i} \mid \forall i \in\{1, \cdots, N\}\right\}$ for all skeleton joints and high order skeleton pattern features $X_{t}^{\prime}=\left\{\mathbf{x}_{t, i}^{\prime} \mid \forall i \in\left\{1, \cdots, N^{\prime}\right\}\right.$ for all discovered patterns.

In addition to predict the action label using LSTM model, we are also interested in predicting which skeleton joints or high order patterns are the most discriminative components for action recognition. Therefore we predict not only action label probabilities $\hat{\mathbf{y}}_{t} \in[0,1]^{K}$, but also skeleton joint attention probabilities $\hat{\mathbf{l}}_{t} \in[0,1]^{N}$, and high order pattern attention probabilities 
$\hat{\mathbf{l}}_{t}^{\prime} \in[0,1]^{N^{\prime}}$, as follows:

$$
\begin{gathered}
\hat{\mathbf{y}}_{t, k}=p\left(Y_{t}=k \mid h_{t}\right)=\frac{\exp \left(A_{k}^{T} h_{t}\right)}{\sum_{k} \exp \left(A_{k}^{T} h_{t}\right)} \\
\hat{\mathbf{l}}_{t, j}=p\left(L_{t}=j \mid h_{t-1}\right)=\frac{\exp \left(B_{j}^{T} h_{t-1}\right)}{\sum_{j} \exp \left(B_{j}^{T} h_{t-1}\right)} \\
\hat{\mathbf{l}}_{t, i}^{\prime}=p\left(L_{t}^{\prime}=i \mid h_{t-1}\right)=\frac{\exp \left(C_{i}^{T} h_{t-1}\right)}{\sum_{i} \exp \left(C_{i}^{T} h_{t-1}\right)}
\end{gathered}
$$

where $A_{k}$ are the weights mapping to the $k$-th action class, $B_{j}$ to the $j$-th skeleton joint, $A_{i}$ to the $i$-th skeleton pattern, and $Y_{t}$ is a random variable which can take one of $K$ action classes, $L_{t}$ can take one of $N$ skeleton joints, $L_{t}^{\prime}$ one of $N^{\prime}$ skeleton patterns.

Following the soft attention model introduced in [86], we compute the input feature vector $\tilde{\mathbf{x}}_{t}$ by computing the expectation of skeleton joint features and skeleton pattern features as follows:

$$
\begin{aligned}
& \mathbf{x}_{t}=E_{p\left(L_{t} \mid h_{t-1}\right)}\left[X_{t}\right]=\sum_{j} \hat{\mathbf{l}}_{t, j} X_{t, j} \\
& \mathbf{x}_{t}^{\prime}=E_{p\left(L_{t}^{\prime} \mid h_{t-1}\right)}\left[X_{t}^{\prime}\right]=\sum_{i} \hat{\mathbf{l}}_{t, i}^{\prime} X_{t, i}^{\prime} \\
& \tilde{\mathbf{x}}_{t}=\left[\mathbf{x}_{t}^{T} ; \mathbf{x}_{t}^{\prime T}\right]^{T}
\end{aligned}
$$

Following the regularizations discussed by [118], we use the cross-entropy loss function to train the attention LSTM model together with $\ell_{2}$-norm regularizations of all model parameters. Additionally, we add regularizations on the attention probabilities learned for skeleton joints and skeleton patterns such that $\sum_{t=1}^{T} \hat{\mathbf{l}}_{t, j} \approx 1$ and $\sum_{t=1}^{T} \hat{\mathbf{l}}_{t, j}^{\prime} \approx 1$, i.e., we impose the constraints that each skeleton joint or skeleton pattern can get the chance of drawing visual attentions over time and thus can prevent some salient joint or pattern dominate the visual attentions all the time. 
The final loss function to train the attention LSTM model is given by:

$$
\begin{aligned}
\text { loss }= & -\sum_{t=1}^{T} \sum_{k=1}^{K} \mathbf{y}_{t, k} \log \hat{\mathbf{y}}_{t, i}+\lambda_{1} \sum_{j=1}^{N}\left(1-\sum_{t=1}^{T} \hat{\mathbf{l}}_{t, j}\right) \\
& +\lambda_{2} \sum_{i=1}^{N^{\prime}}\left(1-\sum_{t=1}^{T} \hat{\mathbf{l}}_{t, i}^{\prime}\right)+\gamma \sum_{i} \sum_{j} \phi_{i, j}^{2}
\end{aligned}
$$

where $\mathbf{y}_{t}$ is the one hot ground truth label vector, $\hat{\mathbf{y}}_{t}$ is the vector of class probabilities at frame $t$, and $T$ is the number of frames, $K$ is the number of classes, $\lambda_{1}$ and $\lambda_{2}$ are the attention penalty coefficient, $\gamma$ is the weight decay coefficient, and $\phi_{i, j}$ represents all the model parameters.

\subsection{Experiments}

In this section, we evaluate our proposed algorithms on MSR Action 3D, MSR Daily Activity 3D, and SBU Interaction datasets. In our experiments, for AND/OR pattern mining, we use up to 100 trees and each tree consists of no more than 8 nodes. For attention LSTM model training, we use 3-layer LSTM models with the number of hidden states and internal cell states set to $512,1024,2048$ and report the best performance. For the attention penalty parameter $\lambda_{1}$ and $\lambda_{2}$, we choose the best parameters from the range $[1,10]$. The model decay weight coefficient is set to $10^{-5}$ and dropout [127] is applied by setting parameter to 0.5 . We train the attention LSTM models up to 300 epochs to evaluate the performances, although it usually converges in 20 epochs. The solver to optimize the attention models is AdamSolver [128]. For all the datasets, we train and test attention LSTM models using 30-frame sliding window data samples with stride set to 1 . The final action label for the video is determined by the average of predictions of all sliding window data samples.

\subsubsection{MSR Action 3D Dataset}

MSR Action 3D dataset [129] is an action dataset of depth sequences captured by a depth camera similar to the Kinect device. It consists of 20 human action classes: high arm wave, 
horizontal arm wave, hammer, hand catch, forward punch, high throw, draw x, draw tick, draw circle, hand clap, two hand wave, side-boxing, bend, forward kick, side kick, jogging, tennis swing, tennis serve, golf swing, pick up throw. Each action was performed by 10 human subjects for 2 to 3 times. We follow the settings in [10] and use 284 sequences for training and 273 sequences for testing.

We report the recognition performances in Table 5.1. From the table we can see that our proposed AND/OR skeleton pattern mining method can significantly improve the recognition performance compared with baselines. For example, we achieve $84.2 \%$ accuracy using linear SVM classifier on the AND/OR pattern features, which significantly outperforms the accuracy $70.7 \%$ on LOP features and $66.7 \%$ on joints features. This result justifies the effectiveness our proposed AND/OR skeleton pattern mining algorithm for action recognition. When comparing the performances between SVM classifier and the proposed attention LSTM model, we find that our attention model can consistently improve from SVM classifier on various features. For example, when using LOP features, we observe $1.8 \%$ improvement from $70.7 \%$ to $72.5 \%$, when using skeleton joint features, we see an improvement of $3.6 \%$ from $66.7 \%$ to $70.3 \%$, and while using the attention LSTM model on the AND/OR skeleton pattern features, our performance goes to $87.9 \%$, which has an improvement of $3.7 \%$ over SVM classifier, and $2.9 \%$ improvement over baseline LSTM model. It is also worth noting that our proposed attention LSTM model can consistent outperform the baseline LSTM model on different skeleton features, e.g., when using LOP features, attention LSTM model achieves 72.5\% while baseline LSTM model only achieved $70.0 \%$, which is an improvement of $2.5 \%$. These results validate the advantages of our proposed attention LSTM model.

When combining attention model with AND/OR skeleton pattern features and the raw skeleton features, we achieve the best performance $91.2 \%$, which has $3.0 \%$ improvement over the previous related work in [10], which also mined high-order skeleton patterns. The result justifies the effectiveness of combining high order skeleton pattern mining and visual attention discovery of our proposed methods. 


\begin{tabular}{l|c}
\hline Algorithm & Accuracy (\%) \\
\hline HFRNN [130] & 42.5 \\
Dynamic temporal warping[131] & 54.0 \\
Hidden Markov model [132] & 63.0 \\
Action graph on bag of 3D points [133] & 74.7 \\
Histogram of 3D joints [134] & 78.9 \\
Random occupancy pattern [114] & 86.2 \\
Eigenjoints [135] & 82.3 \\
Sequence of most informative joints [136] & 47.1 \\
Actionlet [10] & 88.2 \\
\hline SVM on LOP features & 70.7 \\
SVM on joint features & 66.7 \\
SVM on AND/OR patterns & 84.2 \\
\hline LSTM on LOP features & 70.0 \\
LSTM on joint features & 67.8 \\
LSTM on AND/OR patterns & 85.0 \\
\hline Attention LSTM on LOP features & 72.5 \\
Attention LSTM on joint features & 70.3 \\
Attention LSTM on AND/OR patterns & 87.9 \\
Attention LSTM on fusion features & $\mathbf{9 1 . 2}$ \\
\hline
\end{tabular}

TABLE 5.1. Accuracy results on MSR Action 3D dataset.

\subsubsection{MSR Daily Activity 3D Dataset}

MSR Daily Activity 3D [10] dataset is a daily activity dataset that includes the RGB frames, depth sequences and the tracked skeleton joint positions from Microsoft's Kinect camera. It contains in total 16 daily activities from 10 subjects, i.e., drink, eat, read book, call cellphone, write on a paper, use laptop, use vacuum cleaner, cheer up, sit still, toss paper, play game, lay down on sofa, walk, play guitar, stand up, sit down. Each subject performs an activity in two different poses: sitting on sofa and standing. The dataset consists of in total 320 indoor daily activity sequences. In the experiment, we conduct our experiments on the cross-subject test setting, i.e., samples of the subjects $1,2,3,4,5$ are used as training data and samples of the subjects $6,7,8,9,10$ are used as testing data, as in [10].

The recognition accuracy results are shown in Table 5.2. Our proposed attention LSTM model with both discovered AND/OR pattern features and raw skeleton features achieve $88.8 \%$, which has $2.8 \%$ improvement compared with previous actionlet ensemble work [10]. 
In this experiment, we also show the running time comparisons between our proposed branch and bound search method and the exhaustive search method in Fig. 5.4. The x-axis in Fig. 5.4 is the number of input features for pattern mining, and y-axis shows the time spent on mining the optimal AND/OR pattern from labeled dataset. From the figure we can see that, our proposed branch and bound search method can be orders of magnitude faster than the exhaustive search method. For example, our method is around 60 times faster than the exhaustive search method when the input feature dimension is 400 . The running time comparison justifies the efficientness of our proposed AND/OR pattern mining method.

One of the advantages of our AND/OR pattern mining algorithm is that we can visualize which skeleton patterns are the most discriminative for action recognition. In this experiment, we also plot some example AND/OR patterns discovered from MSR Daily Activity 3D dataset in Fig. 5.5.

Thanks to the use of attention model, we can also illustrate where the learned model is focusing and interpret the essentials of human actions by visualization. In Fig. 5.6 we select the most relevant skeleton patterns for action "cheer up" from MSR Daily Activity 3D dataset.

\begin{tabular}{l|c}
\hline Algorithm & Accuracy (\%) \\
\hline Dynamic temporal warping [131] & 54.0 \\
Random occupancy pattern [114] & 64.0 \\
SVM on LOP features [10] & 43.0 \\
SVM on joint features [10] & 68.0 \\
SVM on FTP features [10] & 78.0 \\
Actionlet on LOP features [10] & 61.0 \\
Actionlet on joint features [10] & 74.0 \\
Actionlet on FTP features [10] & 86.0 \\
\hline Attention LSTM on AND/OR patterns & 84.4 \\
Attention LSTM on fusion features & $\mathbf{8 8 . 8}$ \\
\hline
\end{tabular}

TABLE 5.2. Accuracy results of MSR Daily Activity 3D dataset.

\subsubsection{SBU Interaction Dataset}

SBU Interaction dataset [137] contains 8 classes of two-person interactions that are captured with Microsoft Kinect device: approaching, departing, pushing, kicking, punching, exchanging 


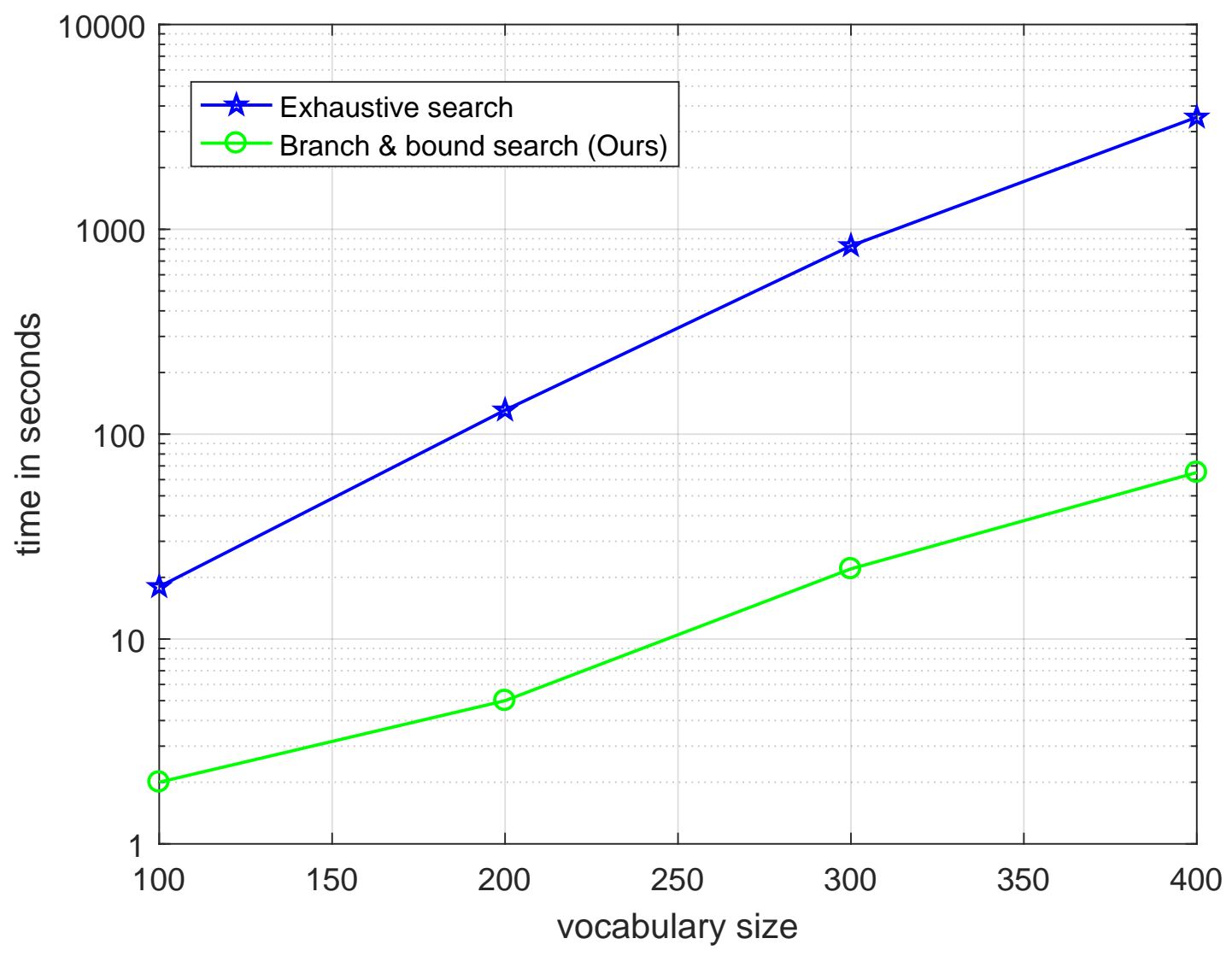

FIGURE 5.4. Running time comparisons between proposed branch and bound search and exhaustive search of high order AND/OR patterns.

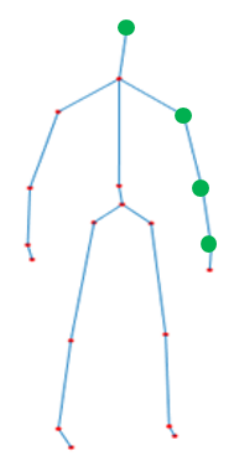

(1)

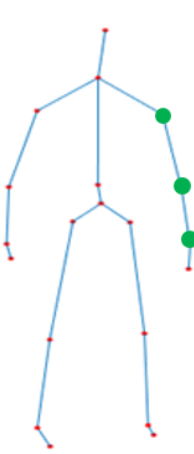

(2)

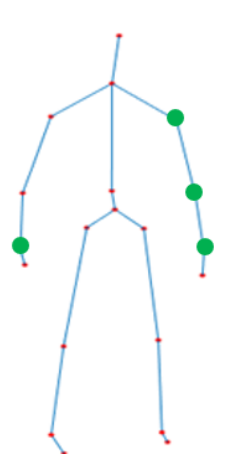

(3)

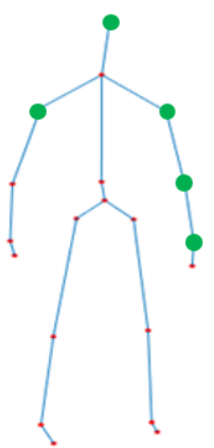

(4)

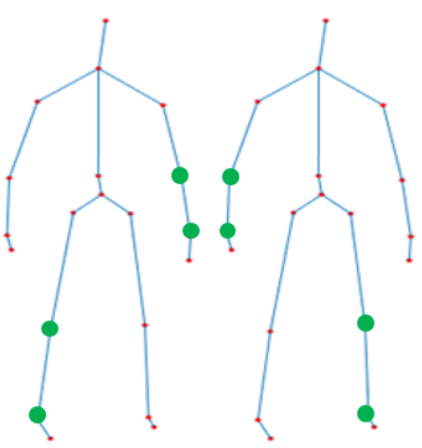

$(5)$

(6)

FIGURE 5.5. Illustration of discovered high order skeleton patterns (in conjunction form) composed by skeleton joints. In the figure, red dots represent raw skeleton joints and green dots represent the selected skeleton joints that form the high order skeleton patterns. (1) and (2) are from action "drink", (3) and (4) are from action "eat", (5)(6) are from action "walk". 


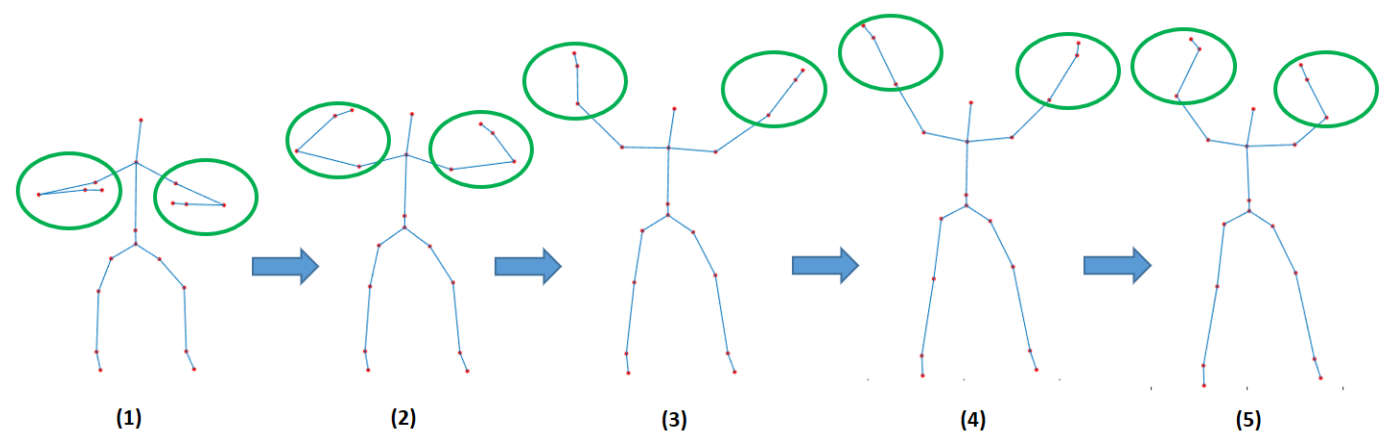

FIGURE 5.6. Illustration of selected visual attentions of high order skeleton patterns for action "cheer up" in 5 frames. We select two skeleton patterns for each frame, based on top two highest coefficients as used in Eq. 5.33, and plot them in green circles. We can see the selected visual attentions over time are quite stable, although there is a change from frame (2) to frame

objects, hugging, and shaking hands. The dataset consists of 282 skeleton sequences in 6822 frames. Each skeleton has 15 joints. This is a more challenging dataset due to the low accuracy of skeleton joints caused by the two-person interactions.

\begin{tabular}{l|c}
\hline Algorithm & Accuracy (\%) \\
\hline Temporal dynamics [138] & 80.3 \\
Interactive contrast mining [139] & 86.9 \\
CHARM [140] & 83.9 \\
HBRNN [115] & 80.4 \\
Co-occurrence LSTM [119] & 90.4 \\
Deep LSTM [119] & 86.0 \\
ST-LSTM (Joint Chain) [116] & 84.7 \\
ST-LSTM (Tree) [116] & 88.6 \\
ST-LSTM (Tree) + Trust Gate [116] & 93.3 \\
\hline Attention LSTM on AND/OR patterns & 86.1 \\
Attention LSTM on fusion features & 91.5 \\
\hline
\end{tabular}

TABLE 5.3. Accuracy results on SBU Interaction dataset.

In this experiment, we compare our proposed methods with more relevant state-of-the-art LSTM models such as co-occurrence LSTM [119] and ST-LSTM [116]. The recognition results are reported in Table 5.3. As can be seen from the table, our attention LSTM model with discovered AND/OR patterns can achieve $86.1 \%$, which has $1.4 \%$ improvement over the ST-LSTM using Joint Chain [116]. We also find that when using both skeleton pattern features and skeleton joint features, our method achieves $91.5 \%$ accuracy, which is $1.1 \%$ better compared with 
the co-occurrence LSTM work [119]. Although our final performance is not as good as the best performance $93.3 \%$ in ST-LSTM [116], it is worth mentioning that they had applied more complex tree structure in the LSTM model while we only use the simple chain structure.

\subsubsection{NTU RGBD Dataset}

NTU-RGBD dataset [141] is a large scale dataset for 3D action recognition. There are around 56 thousand sequences with 60 action classes, which are performed by 40 subjects. In the dataset, each skeleton has 25 skeleton joints. We follow the experiment protocol in [141] in the experiments. There are two settings for the evaluation, one is cross-subject (half subjects for training and half for testing) and the other is cross-view (2/3 views for training and $1 / 3$ views for testing). We report the results in Table 5.4, from which we can see that our methods can outperform the baseline method [141] by $2.3 \%$ under cross-subject setting and $4.6 \%$ under cross-view setting. Although our proposed method does not outperform the ST-LSTM model with trust gate method [116], it is worth noting that our LSTM model is of simple chain structure in temporal domain while [116] uses more complex tree structure to model both spatial and temporal context information.

\begin{tabular}{l|c|c}
\hline Algorithm & Cross-Subject (\%) & Cross-View (\%) \\
\hline Lie group [142] & 50.1 & 52.8 \\
HBRNN [115] & 59.1 & 64.0 \\
Part-Aware LSTM [141] & 62.9 & 70.3 \\
ST-LSTM (Tree) + Trust Gate [116] & $\mathbf{6 9 . 2}$ & $\mathbf{7 7 . 7}$ \\
\hline Attention LSTM on AND/OR patterns & 63.8 & 72.5 \\
Attention LSTM on fusion features & 65.2 & 74.9 \\
\hline
\end{tabular}

TABLE 5.4. Accuracy results on NTU-RGBD dataset.

\subsubsection{Conclusion and Future Work}

Human actions can be characterized by motions of skeleton joints or groups of skeleton joints. We propose to learn visual attentions of high order AND/OR skeleton patterns to exploit both 
spatial and temporal context information for action recognition. In the spatial domain, our proposed method can efficiently mine discriminative high order AND/OR patterns of skeleton joints by branch and bound search. In the temporal domain, our proposed method can learn the visual attentions over time by focusing selectively on parts of skeletons that are relevant for the action recognition task at hand. Experiments on MSR Action 3D, MSR Daily Activity 3D, SBU interaction and NTU-RGBD datasets justify both effectiveness and efficientness of our proposed methods.

There are still many potential extensions to this work. For example, as shown in the experiments, the ST-LSTM model [116] that utilizes tree structure to exploit both spatial and temporal context information achieved promising performances for 3D action recognition, it would be interesting to borrow such tree structure in our attention model to further improve action recognition performance and also discover skeleton patterns of our interests.

Another extension of this work would be trying to integrate the spatial pattern mining process into the temporal attention model by end-to-end learning.

It would also be interesting if we can apply the attention mechanism in the spatial domain considering that the attention mechanism is only applied in temporal domain in our current method. 



\section{Chapter 6}

\section{Conclusion and Future Work}

\subsection{Conclusion}

We have explored the problem of supervised visual pattern discovery for image and video data analysis. We have focused on (1) supervised discovery of mid-level visual phrases from local spatial context aware and multi-feature fused visual primitives; (2) supervised discovery of class-specific spatial layout patterns for scene recognition; (3) supervised discovery of optimal high order AND/OR patterns of individual features.

To justify the advantages of supervised discovery of spatial context aware visual pattern from image data, we have shown in Chapter 2 that local spatial neighborhood context plays an important role in helping construct representative mid-level visual phrases based on low-level visual primitives for applications such as texture pattern discovery, scene image clustering. Specifically, we build a hierarchical architecture to discover visual patterns, i.e., the mid-level spatial context aware and multi-feature fused visual phrases, and utilize back propagation technique to learn the sparse codes and dictionary for the visual patterns by leveraging weakly-supervised image label information.

In Chapter 3 we show how to discover visual patterns of class-specific spatial layouts for each scene category from labeled scene recognition dataset. Specifically, we propose to jointly learn 
the weights of different spatial layouts and image classifiers by casting a max-margin optimization problem. Experiments show that the discovered class-specific spatial layouts can better represent scene images and help improve scene recognition performances compared to previous methods that either use class-generic spatial layouts or use pre-defined spatial layouts.

To illustrate the effectiveness and efficiency of supervised mining AND/OR co-occurrence patterns from labeled transaction dataset, we have demonstrated in Chapter 4 and 5, both in theory and practice, effective and efficient solutions with clear performance gains to mine cooccurrence patterns of individual features from weighted and labeled dataset. Specifically, we propose a branch-and-bound search algorithm to efficiently mine the optimal co-occurrence pattern from weighted and labeled dataset. The discovered co-occurrence patterns can help achieve superior performance than algorithm using raw features directly.

\subsection{Future Work}

The approaches proposed in this thesis can be improved in many ways. We describes some attempts as follows.

\subsubsection{Discovering Visual Patterns from Convolution Neural Networks}

In Chapter 4, our proposed pattern mining algorithm is applied on human defined visual features, however it will be more interesting to discover visual patterns from deep learning features. As proved by many recent works, deep learning features learned from large scale dataset can significantly outperform human defined features. For example, convolution neural network $(\mathrm{CNN})$ methods have been very popular for visual recognition in recent years due to the stateof-the-art performances [51, 53, 54, 68]. As discussed in [143], feature maps in different CNN layers have different semantic meanings, e.g., some responds to edge/corners, some captures similar textures etc. Therefore as a natural extension of our work in Chapter 4, it would be interesting to discover co-occurrence patterns from multi-layer $\mathrm{CNN}$ feature maps for visual 
recognition. The conjunction and disjunction of different feature maps from different CNN layers can help capture more semantic features for visual recognition.

\subsubsection{Discovering Class-specific Temporal Layout Patterns for Action Recog- nition}

As shown in Fig. 3.1 in Chapter 3, different scene categories have large inter-class variations of spatial layouts, and even the same scene category also have large intra-class variations of spatial layouts. The similar observations go to human actions. Different action categories can have different performing speeds and thus different temporal layouts. Also within the same action category, different human subjects can carry out different performing speeds. Therefore it is worth to exploit how to discover class-specific visual patterns of temporal layouts for action recognition, as an extension of our work in Chapter 3.

\subsubsection{Learning Attention LSTM Model with Tree Structure}

In Chapter 5, as shown in Fig. 5.3, we propose our LSTM model in linear chain structure. However linear chain structure can only exploit temporal context information. As discussed in [116], LSTM model based on tree structure can exploit both spatial context and temporal context information and has been proved to outperform LSTM model with linear chain structure. Therefore as an extension of our work in Chapter 5, we can develop our attention LSTM model based on tree structure to take advantage of both spatial context and temporal context information to improve action recognition performance. 



\section{Author's Publications}

- Junwu Weng, Chaoqun Weng, and Junsong Yuan, Spatio-Temporal Naive-Bayes NearestNeighbor (ST-NBNN) for Skeleton-Based Action Recognition, in Proc. IEEE Conf. on Computer Vision Pattern Recognition (CVPR'17), 2017.

- Chaoqun Weng, Hongxing Wang, Junsong Yuan, and Xudong Jiang. Discovering ClassSpecific Spatial Layouts for Scene Recognition. Signal Processing Letters (SPL), 2016.

- Hongxing Wang, Yoshinobu Kawahara, Chaoqun Weng, Junsong Yuan. Representative Selection with Structured Sparsity. Pattern recognition (PR), 2016.

- Michael Hoy, Chaoqun Weng, Junsong Yuan, and Justin Dauwels. Bayesian Tracking of Multiple Objects with Vision and Radar, in Intl. Conf. on Control, Automation, Robotics and Vision (ICARCV), 2016.

- Michael Hoy, Chaoqun Weng, Junsong Yuan, and Justin Dauwels. Object Detection and Tracking with Multiple Sensor Modalities using Random Finite Set Filtering, in workshop of Intl. Conf. on Autonomous Agents and Multiagent Systems (AAMAS), 2016.

- Chaoqun Weng, Hongxing Wang, and Junsong Yuan. Efficient Mining of Optimal AND/OR Patterns for Visual Recognition, IEEE Trans. on Multimedia (TMM), 2015.

- Hongxing Wang, Chaoqun Weng, Junsong Yuan. Multi-feature Spectral Clustering with Minimax Optimization, in Proc. IEEE Conf. on Computer Vision Pattern Recognition (CVPR), 2014. 
- Chaoqun Weng, Yuning Jiang, and Junsong Yuan. Direct Mining Co-occurrence Features for Visual Recognition: A Branch and Bound Method, in Proc. IEEE Conf. on Multimedia Expo (ICME), 2013 (oral, acceptance rate [15\%]).

- Chaoqun Weng, Hongxing Wang, and Junsong Yuan. Hierarchical Sparse Coding based on Spatial Pooling and Multi-feature Fusion, in Proc. IEEE Conf. on Multimedia Expo (ICME), 2013.

- Chaoqun Weng, Hongxing Wang, and Junsong Yuan. Learning Weighted Geometric Pooling for Image Classification, in Proc. IEEE Conf. on Image Processing (ICIP), 2013.

- Chaoqun Weng, Gang Hua, and Junsong Yuan. Multiple Instance Boosting with Global Smoothness Regularization, in Intl. Conf. on Information, Communication and Signal Processing (ICICS), 2011. 


\section{Bibliography}

[1] D. Lowe, "Distinctive image features from scale-invariant keypoints," International Journal of Computer Vision (IJCV), 2004.

[2] J. Winn, A. Criminisi, and T. Minka, “Object categorization by learned universal visual dictionary," in IEEE International Conference on Computer Vision (ICCV), 2005.

[3] J. Yuan, M. Yang, and Y. Wu, "Mining discriminative co-occurrence patterns for visual recognition," in IEEE Conference on Computer Vision and Pattern Recognition (CVPR), 2011.

[4] J. Yuan and Y. Wu, "Spatial random partition for common visual pattern discovery," in 2007 IEEE 11th International Conference on Computer Vision. IEEE, 2007, pp. 1-8.

[5] H. Liu and S. Yan, "Common visual pattern discovery via spatially coherent correspondences," in Computer Vision and Pattern Recognition (CVPR), 2010 IEEE Conference on. IEEE, 2010, pp. 1609-1616.

[6] H. Xie, K. Gao, Y. Zhang, J. Li, and H. Ren, "Common visual pattern discovery via graph matching," in Proceedings of the 19th ACM international conference on multimedia. ACM, 2011, pp. 1385-1388.

[7] G. Zhao and J. Yuan, "Mining and cropping common objects from images," in Proceedings of the 18th ACM International Conference on Multimedia, ser. MM '10. New York, NY, USA: ACM, 2010, pp. 975-978. [Online]. Available: http://doi.acm.org/10.1145/1873951.1874127 
[8] S. Nowozin, K. Tsuda, T. Uno, T. Kudo, and G. BakIr, "Weighted substructure mining for image analysis," in 2007 IEEE Conference on Computer Vision and Pattern Recognition. IEEE, 2007, pp. 1-8.

[9] S. Nowozin, G. Bakir, and K. Tsuda, "Discriminative subsequence mining for action classification," in 2007 IEEE 11th International Conference on Computer Vision. IEEE, 2007, pp. 1-8.

[10] J. Wang, Z. Liu, Y. Wu, and J. Yuan, "Learning actionlet ensemble for 3d human action recognition," IEEE Transactions on Pattern Analysis and Machine Intelligence (TPAMI), 2014.

[11] P. Wang, W. Li, P. Ogunbona, Z. Gao, and H. Zhang, "Mining mid-level features for action recognition based on effective skeleton representation," in Digital lmage Computing: Techniques and Applications (DlCTA), 2014 International Conference on. IEEE, 2014, pp. 1-8.

[12] R. Agrawal and R. Srikant, "Fast algorithms for mining association rules in large databases," in International Conference on Very Large Data Bases (VLDB), 1994.

[13] J. Han, J. Pei, and Y. Yin, "Mining frequent patterns without candidate generation," in ACM SIGMOD Record, 2000.

[14] H. Cheng, X. Yan, J. Han, and C.-W. Hsu, "Discriminative frequent pattern analysis for effective classification," in 2007 IEEE 23rd International Conference on Data Engineering. IEEE, 2007, pp. 716-725.

[15] H. Cheng, X. Yan, J. Han, and S. Y. Philip, "Direct discriminative pattern mining for effective classification," in 2008 IEEE 24th International Conference on Data Engineering. IEEE, 2008, pp. 169-178.

[16] J. Feng, B. Ni, Q. Tian, and S. Yan, "Geometric lp-norm feature pooling for image classification," in IEEE Conference on Computer Vision and Pattern Recognition (CVPR), 2011. 
[17] Y. Zhang, Z. Jia, and T. Chen, "Image retrieval with geometry-preserving visual phrases," in IEEE Conference on Computer Vision and Pattern Recognition (CVPR), 2011.

[18] Y. Lu, L. Zhang, Q. Tian, and W. Ma, "What are the high-level concepts with smal1 semantic gaps?" in IEEE Conference on Computer Vision and Pattern Recognition (CVPR), 2008.

[19] A. Kumar, P. Rai, and H. Daume, "Co-regularized multi-view spectral clustering," in Advances in neural information processing systems, 2011, pp. 1413-1421.

[20] X. Cai, F. Nie, H. Huang, and F. Kamangar, "Heterogeneous image feature integration via multi-modal spectral clustering," in Computer Vision and Pattern Recognition (CVPR), 2011 IEEE Conference on. IEEE, 2011, pp. 1977-1984.

[21] H. Wang, C. Weng, and J. Yuan, "Multi-feature spectral clustering with minimax optimization," in Proceedings of the IEEE Conference on Computer Vision and Pattern Recognition, 2014, pp. 4106-4113.

[22] S. Lazebnik, C. Schmid, and J. Ponce, "Beyond bags of features: Spatial pyramid matching for recognizing natural scene categories," in IEEE Conference on Computer Vision and Pattern Recognition (CVPR), 2006.

[23] J. Yang, K. Yu, Y. Gong, and T. Huang, "Linear spatial pyramid matching using sparse coding for image classification," in IEEE Conference on Computer Vision and Pattern Recognition (CVPR), 2009.

[24] K. He, X. Zhang, S. Ren, and J. Sun, "Spatial pyramid pooling in deep convolutional networks for visual recognition," in European Conference on Computer Vision (ECCV), 2014, pp. 346-361.

[25] J. Sivic and A. Zisserman, "Video google: A text retrieval approach to object matching in videos," in IEEE International Conference on Computer Vision (ICCV), 2003. 
[26] J. Yuan, Y. Wu, and M. Yang, "Discovery of collocation patterns: from visual words to visual phrases," in IEEE Conference on Computer Vision and Pattern Recognition (CVPR), 2007.

[27] Y. Boureau, F. Bach, Y. LeCun, and J. Ponce, "Learning mid-level features for recognition,” in IEEE Conference on Computer Vision and Pattern Recognition (CVPR), 2010.

[28] D. Liu, G. Hua, P. Viola, and T. Chen, "Integrated feature selection and higher-order spatial feature extraction for object categorization," in IEEE Conference on Computer Vision and Pattern Recognition (CVPR), 2008.

[29] M. A. Sadeghi and A. Farhadi, "Recognition using visual phrases." in IEEE Conference on Computer Vision and Pattern Recognition (CVPR), 2011.

[30] S. Zhang, Q. Tian, G. Hua, Q. Huang, and W. Gao, "Generating descriptive visual word$\mathrm{s}$ and visual phrases for large-scale image applications," IEEE Transactions on Image Processing (TIP), vol. 20, no. 9, pp. 2664-2677, 2011.

[31] S. Zhang, Q. Tian, Q. Huang, W. Gao, and Y. Rui, "Multi-order visual phrase for scalable partial-duplicate visual search," Multimedia Systems, vol. 21, no. 2, pp. 229-241, 2014.

[32] J. Wang, J. Yang, K. Yu, F. Lv, T. Huang, and Y. Gong, "Locality-constrained linear coding for image classification," in IEEE Conference on Computer Vision and Pattern Recognition (CVPR), 2010.

[33] F. Perronnin, J. Snchez, and T. Mensink, "Improving the fisher kernel for large-scale image classification," in European Conference on Computer Vision (ECCV), 2010.

[34] X.-C. Lian, Z. Li, B.-L. Lu, and L. Zhang, "Max-margin dictionary learning for multiclass image categorization," in European Conference on Computer Vision (ECCV), 2010.

[35] L. Yang, R. Jin, R. Sukthankar, and F. Jurie, "Unifying discriminative visual codebook generation with classifier training for object category recognition," in IEEE Conference on Computer Vision and Pattern Recognition (CVPR), 2008. 
[36] Z. Jiang, Z. Lin, and L. Davis, "Learning a discriminative dictionary for sparse coding via label consistent k-svd," in IEEE Conference on Computer Vision and Pattern Recognition (CVPR), 2011.

[37] Z. Jiang, G. Zhang, and L. S. Davis, "Submodular dictionary learning for sparse coding," in IEEE Conference on Computer Vision and Pattern Recognition (CVPR), 2012.

[38] F. Zhu and L. Shao, "Enhancing action recognition by cross-domain dictionary learning," in British Machine Vision Conference (BMVC), 2013.

[39] V. Sydorov, M. Sakurada, and C. H. Lampert, "Deep fisher kernels - end to end learning of the fisher kernel gmm parameters," in IEEE Conference on Computer Vision and Pattern Recognition (CVPR), 2014.

[40] J. Yuan and Y. Wu, "Context-aware clustering," in IEEE Conference on Computer Vision and Pattern Recognition (CVPR), 2008.

[41] H. Wang, J. Yuan, and Y.-P. Tan, "Combining feature context and spatial context for image pattern discovery," in The IEEE International Conference on Data Mining series (ICDM), 2011.

[42] H. Wang, J. Yuan, and Y. Wu, "Context-aware discovery of visual co-occurrence patterns," IEEE Transactions on Image Processing (TIP), vol. 23, no. 4, pp. 1805-1819, 2014.

[43] J. Wang, J. Yuan, Z. Chen, and Y. Wu, "Spatial locality-aware sparse coding and dictionary learning," in The Asian Conference on Machine Learning (ACML), 2012.

[44] S. Zhang, Q. Tian, G. Hua, Q. Huang, and S. Li, "Descriptive visual words and visual phrases for image applications," in ACM Multimedia, 2009.

[45] P. V. Gehler and S. Nowozin, "On feature combination for multiclass object classification." in IEEE International Conference on Computer Vision (ICCV), 2009. 
[46] H. Ma, J. Zhu, M.-T. Lyu, and I. King, "Bridging the semantic gap between image contents and tags," IEEE Transactions on Multimedia (TMM), vol. 12, no. 5, pp. 462473, 2010.

[47] L. Xie, Q. Tian, M. Wang, and B. Zhang, "Spatial pooling of heterogeneous features for image classification," IEEE Transactions on Image Processing (TIP), vol. 23, no. 5, pp. 1994-2008, 2014.

[48] Y. Yang, J. Song, Z. Huang, Z. Ma, N. Sebe, and A. G. Hauptmann, "Multi-feature fusion via hierarchical regression for multimedia analysis." IEEE Transactions on Multimedia (TMM), vol. 15, no. 3, pp. 572-581, 2013.

[49] G. E. Hinton, S. Osindero, and Y. W. Teh, "A fast learning algorithm for deep belief nets," Neural Computation, vol. 18, pp. 1527-1554, 2006.

[50] H. Lee, R. B. Grosse, R. Ranganath, and A. Y. Ng, "Convolutional deep belief networks for scalable unsupervised learning of hierarchical representations." in The International Conference on Machine Learning (ICML), 2009.

[51] A. Krizhevsky, I. Sutskever, and G. E. Hinton, "Imagenet classification with deep convolutional neural networks," in Advances in neural information processing systems (NIPS), 2012, pp. 1097-1105.

[52] K. Simonyan and A. Zisserman, "Very deep convolutional networks for large-scale image recognition," CoRR, vol. abs/1409.1556, 2014.

[53] B. Zhou, A. Lapedriza, J. Xiao, A. Torralba, and A. Oliva, "Learning deep features for scene recognition using places database," in Advances in neural information processing systems (NIPS), 2014, pp. 487-495.

[54] K. He, X. Zhang, S. Ren, and J. Sun, “Deep residual learning for image recognition,” in IEEE Conference on Computer Vision and Pattern Recognition (CVPR), 2016. 
[55] K. Chatfield, K. Simonyan, A. Vedaldi, and A. Zisserman, "Return of the devil in the details: Delving deep into convolutional nets." in British Machine Vision Conference (BMVC), 2014.

[56] K. Yu, Y. Lin, and J. Lafferty, "Learning image representations from the pixel level via hierarchical sparse coding," in IEEE Conference on Computer Vision and Pattern Recognition (CVPR), 2011.

[57] H. Lee, A. Battle, R. Raina, and A. Ng, "Efficient sparse coding algorithms," in Advances in neural information processing systems (NIPS), 2006.

[58] D. M. Bradley and J. Bagnell, "Differentiable sparse coding," in Advances in neural information processing systems (NIPS), 2008.

[59] T. Malisiewicz and A. Efros, "Improving spatial support for objects via multiple segmentations," in British Machine Vision Conference (BMVC), 2007.

[60] Y. Lee and K. Grauman, "Object-graphs for context-aware visual category discovery," IEEE Transactions on Pattern Analysis and Machine Intelligence (TPAMI), 2012.

[61] A. Bosch, A. Zisserman, and X. Munoz, "Representing shape with a spatial pyramid kernel," in The International Conference on Image and Video Retrieval (CIVR), 2007.

[62] Y. Yang, H. T. Shen, Z. Ma, Z. Huang, and X. Zhou, "L2,1-norm regularized discriminative feature selection for unsupervised learning," in International Joint Conference on Artificial Intelligence (IJCAI), 2011.

[63] A. Oliva and A. Torralba, "Modeling the shape of the scene: A holistic representation of the spatial envelope," International Journal of Computer Vision (IJCV), 2001.

[64] J. van Gemert, J. Geusebroek, C. Veenman, and A. Smeulders, "Kernel codebooks for scene categorization,” in European Conference on Computer Vision (ECCV), 2008.

[65] L. Liu, L. Wang, and X. Liu, "In defense of soft-assignment coding," in IEEE International Conference on Computer Vision (ICCV), 2011. 
[66] L.-J. Li, H. Su, E. P. Xing, and F.-F. Li, “Object bank: A high-level image representation for scene classification and semantic feature sparsification." in Advances in neural information processing systems (NIPS), 2010.

[67] L. Bo, X. Ren, and D. Fox, "Kernel descriptors for visual recognition," in Advances in neural information processing systems (NIPS), 2010.

[68] S. Ren, K. He, R. Girshick, and J. Sun, "Faster r-cnn: Towards real-time object detection with region proposal networks," in Advances in neural information processing systems (NIPS), 2015, pp. 91-99.

[69] Y. Jiang, J. Yuan, and G. Yu, "Randomized spatial partition for scene recognition," in European Conference on Computer Vision (ECCV), 2012.

[70] J. Ren, X. Jiang, J. Yuan, and G. Wang, “Optimizing lbp structure for visual recognition using binary quadratic programming," IEEE Signal Processing Letters, vol. 21, no. 11, pp. 1346-1350, 2014.

[71] Z. Zuo and G. Wang, "Learning discriminative hierarchical features for object recognition,” IEEE Signal Processing Letters, vol. 21, no. 9, pp. 1159-1163, Sept 2014.

[72] B. Shuai, Z. Zuo, and G. Wang, "Quaddirectional 2d-recurrent neural networks for image labeling," IEEE Signal Processing Letters, vol. 22, no. 11, pp. 1990-1994, Nov 2015.

[73] D. Tao, X. Li, X. Wu, W. Hu, and S. Maybank, “Supervised tensor learning," Knowledge and Information Systems, 2007.

[74] J. Wu and J. M. Rehg, "Centrist: A visual descriptor for scene categorization," IEEE Transactions on Pattern Analysis and Machine Intelligence (TPAMI), 2011.

[75] R.-E. Fan, K.-W. Chang, C.-J. Hsieh, X.-R. Wang, and C.-J. Lin, "Liblinear: A library for large linear classification," Journal of machine learning research, vol. 9, no. Aug, pp. 1871-1874, 2008. 
[76] C. Cortes and V. Vapnik, "Support-vector networks," Machine learning, vol. 20, no. 3, pp. 273-297, 1995.

[77] D. L. Donoho, "For most large underdetermined systems of linear equations the minimal 11-norm solution is also the sparsest solution," Communications on pure and applied mathematics, vol. 59, no. 6, pp. 797-829, 2006.

[78] K. Crammer and Y. Singer, "On the algorithmic implementation of multiclass kernelbased vector machines," J. Mach. Learn. Res., vol. 2, pp. 265-292, Mar. 2002.

[79] C.-W. Hsu and C.-J. Lin, "A comparison of methods for multiclass support vector machines," IEEE Transactions on Neural Networks, vol. 13, no. 2, pp. 415-425, 2002.

[80] K.-B. Duan and S. S. Keerthi, "Which is the best multiclass svm method? an empirical study," in International Workshop on Multiple Classifier Systems, 2005, pp. 278-285.

[81] Y. Yang and S. Newsam, "Spatial pyramid co-occurrence for image classification," in IEEE International Conference on Computer Vision (ICCV), 2011.

[82] A. Quattoni and A. Torralba, "Recognizing indoor scenes," in IEEE Conference on Computer Vision and Pattern Recognition (CVPR), 2009, pp. 413-420.

[83] J. Sun and J. Ponce, "Learning discriminative part detectors for image classification and cosegmentation," in IEEE International Conference on Computer Vision (ICCV), 2013, pp. 3400-3407.

[84] M. Juneja, A. Vedaldi, C. Jawahar, and A. Zisserman, "Blocks that shout: Distinctive parts for scene classification," in IEEE Conference on Computer Vision and Pattern Recognition (CVPR'13), 2013, pp. 923-930.

[85] C. Doersch, A. Gupta, and A. A. Efros, "Mid-level visual element discovery as discriminative mode seeking," in Advances in neural information processing systems (NIPS), 2013, pp. 494-502. 
[86] W. Zaremba, I. Sutskever, and O. Vinyals, "Recurrent neural network regularization," arXiv preprint arXiv:1409.2329, 2014.

[87] A. Farhadi, I. Endres, D. Hoiem, and D. Forsyth, "Describing objects by their attributes," in IEEE Conference on Computer Vision and Pattern Recognition (CVPR), 2009.

[88] P. Dollar, Z. Tu, H. Tao, and S. Belongie, "Feature mining for image classification," in IEEE Conference on Computer Vision and Pattern Recognition (CVPR), 2007.

[89] T. Mita, T. Kaneko, B. Stenger, and O. Hori, "Discriminative feature co-occurrence selection for object detection," IEEE Transactions on Pattern Analysis and Machine Intelligence (TPAMI), 2008.

[90] S. Ito and S. Kubota, "Object classification using heterogeneous co-occurrence features," in European Conference on Computer Vision (ECCV), 2010.

[91] J. Yuan, J. Luo, and Y. Wu, "Mining compositional features from gps and visual cues for event recognition in photo collections," IEEE Transactions on Multimedia (TMM), 2010.

[92] S. Tsai, L. Cao, F. Tang, and T. Huang, "Compositional object pattern: A new model for album event recognition," in ACM Multimedia, 2011.

[93] B. Fernando, E. Fromont, and T. Tuytelaars, "Mining mid-level features for image classification," International Journal of Computer Vision (IJCV), 2014.

[94] B. C. Winn Voravuthikunchai and F. Jurie, "Histograms of pattern sets for image classification and object recognition," in IEEE Conference on Computer Vision and Pattern Recognition (CVPR), 2014.

[95] P. K. Novak, N. Lavrač, and G. I. Webb, "Supervised descriptive rule discovery: A unifying survey of contrast set, emerging pattern and subgroup mining," The Journal of Machine Learning Research (JMLR), 2009. 
[96] A. Torralba, K. P. Murphy, and W. T. Freeman, "Sharing visual features for multiclass and multiview object detection," IEEE Transactions on Pattern Analysis and Machine Intelligence (TPAMI), 2007.

[97] G. Wang and D. Forsyth, "Joint learning of visual attributes, object classes and visual saliency," in IEEE International Conference on Computer Vision (ICCV), 2009.

[98] J. Yuan, J. Luo, and Y. Wu, "Mining compositional features for boosting," in IEEE Conference on Computer Vision and Pattern Recognition (CVPR), 2008.

[99] R. E. Schapire and Y. Singer, "Improved boosting algorithms using confidence-rated predictions," Machine learning, 1999.

[100] Y. Freund and R. E. Schapire, "A decision-theoretic generalization of on-line learning and an application to boosting," Journal of Computer and System Sciences, 1997.

[101] B. Kégl and R. Busa-Fekete, "Boosting products of base classifiers," in The International Conference on Machine Learning (ICML), 2009.

[102] O. Danielsson, S. Carlsson, and J. Sullivan, "Automatic learning and extraction of multilocal features," in IEEE International Conference on Computer Vision (ICCV), 2009.

[103] B. Yao and F. Li, "Grouplet: A structured image representation for recognizing human and object interactions," in IEEE Conference on Computer Vision and Pattern Recognition (CVPR), 2010.

[104] Y. Jiang, J. Meng, and J. Yuan, "Randomized visual phrase for object search," in IEEE Conference on Computer Vision and Pattern Recognition (CVPR), 2012.

[105] S. Zhang, Q. Huang, G. Hua, S. Jiang, W. Gao, and Q. Tian, "Building contextual visual vocabulary for large-scale image applications," in ACM Multimedia, 2010.

[106] S. Nowozin, K. Tsuda, T. Uno, T. Kudo, and G. BakIr, "Weighted substructure mining for image analysis," in IEEE Conference on Computer Vision and Pattern Recognition (CVPR), 2007. 
[107] S. Nowozin, G. Bakir, and K. Tsuda, "Discriminative subsequence mining for action classification," in IEEE International Conference on Computer Vision (ICCV), 2007.

[108] T. Ahonen, A. Hadid, and M. Pietikinen, "Face recognition with local binary patterns," in European Conference on Computer Vision (ECCV), 2004.

[109] N. Dalal and B. Triggs, "Histograms of oriented gradients for human detection," in IEEE Conference on Computer Vision and Pattern Recognition (CVPR), 2005.

[110] K. Yu, T. Zhang, and Y. Gong, "Nonlinear learning using local coordinate coding," in Advances in neural information processing systems (NIPS), 2009.

[111] J. Li and M. Shah, "Scene modeling using co-clustering," in IEEE International Conference on Computer Vision (ICCV), 2007.

[112] A. Bosc, A. Zisserman, and X. Munoz, "Scene classification using a hybrid generative/discriminative approach," IEEE Transactions on Pattern Analysis and Machine Intelligence (TPAMI), 2008.

[113] M. Müller and T. Röder, "Motion templates for automatic classification and retrieval of motion capture data," in ACM SIGGRAPH, 2006.

[114] J. Wang, Z. Liu, J. Chorowski, Z. Chen, and Y. Wu, "Robust 3d action recognition with random occupancy patterns," in Computer vision-ECCV 2012, 2012, pp. 872-885.

[115] Y. Du, W. Wang, and L. Wang, "Hierarchical recurrent neural network for skeleton based action recognition," in 2015 IEEE Conference on Computer Vision and Pattern Recognition (CVPR), 2015, pp. 1110-1118.

[116] J. Liu, A. Shahroudy, D. Xu, and G. Wang, "Spatio-temporal LSTM with trust gates for 3d human action recognition," 2016.

[117] V. Veeriah, N. Zhuang, and G.-J. Qi, "Differential recurrent neural networks for action recognition," in Proceedings of the IEEE International Conference on Computer Vision, 2015, pp. 4041-4049. 
[118] K. Xu, J. Ba, R. Kiros, K. Cho, A. Courville, R. Salakhutdinov, R. S. Zemel, and Y. Bengio, "Show, attend and tell: Neural image caption generation with visual attention," 2015.

[119] W. Zhu, C. Lan, J. Xing, W. Zeng, Y. Li, L. Shen, and X. Xie, "Co-occurrence feature learning for skeleton based action recognition using regularized deep LSTM networks," 2016.

[120] J. Shotton, T. Sharp, A. Kipman, A. Fitzgibbon, M. Finocchio, A. Blake, M. Cook, and R. Moore, "Real-time human pose recognition in parts from single depth images," Communications of the ACM, vol. 56, no. 1, pp. 116-124, 2013.

[121] P. Wang, W. Li, P. Ogunbona, Z. Gao, and H. Zhang, "Mining mid-level features for action recognition based on effective skeleton representation," CoRR, vol. abs/1409.4014, 2014.

[122] S. Park, B. X. Nie, and S. Zhu, "Attribute and-or grammar for joint parsing of human attributes, part and pose," CoRR, vol. abs/1605.02112, 2016.

[123] Y. N. W. Quanshi Zhang, Ruiming Cao and S.-C. Zhu, "Growing interpretable part graphs on convnets via multi-shot learning," in The AAAI Conference on Artificial Intelligence (AAAI), 2017.

[124] Q. Zhang, Y. Nian Wu, and S.-C. Zhu, "Mining and-or graphs for graph matching and object discovery," in IEEE International Conference on Computer Vision (ICCV), 2015, pp. 55-63.

[125] L. Breiman, “Random forests," Machine learning, vol. 45, no. 1, pp. 5-32, 2001.

[126] A. Graves, "Supervised sequence labelling," in Supervised Sequence Labelling with Recurrent Neural Networks. Springer, 2012, pp. 5-13.

[127] N. Srivastava, G. E. Hinton, A. Krizhevsky, I. Sutskever, and R. Salakhutdinov, "Dropout: a simple way to prevent neural networks from overfitting." Journal of Machine Learning Research, vol. 15, no. 1, pp. 1929-1958, 2014. 
[128] J. Ba, V. Mnih, and K. Kavukcuoglu, "Multiple object recognition with visual attention," International Conference on Learning Representations (ICLR), 2015.

[129] W. Li, Z. Zhang, and Z. Liu, "Action recognition based on a bag of 3d points," in 2010 IEEE Computer Society Conference on Computer Vision and Pattern RecognitionWorkshops. IEEE, 2010, pp. 9-14.

[130] J. Martens and I. Sutskever, "Learning recurrent neural networks with hessian-free optimization," in International Conference on Machine Learning (ICML), 2011, pp. 10331040.

[131] M. Müller and T. Röder, "Motion templates for automatic classification and retrieval of motion capture data," in ACM SIGGRAPH/Eurographics Symposium on Computer Animation, ser. SCA ’06, 2006, pp. 137-146.

[132] F. Lv and R. Nevatia, "Recognition and segmentation of 3-d human action using hmm and multi-class adaboost," in European Conference on Computer Vision, ser. ECCV, 2006, pp. 359-372.

[133] W. Li, Z. Zhang, and Z. Liu, "Action recognition based on a bag of 3d points," in IEEE Computer Society Conference on Computer Vision and Pattern Recognition-Workshops. IEEE, 2010, pp. 9-14.

[134] L. Xia, C.-C. Chen, and J. Aggarwal, "View invariant human action recognition using histograms of 3d joints," in IEEE Computer Society Conference on Computer Vision and Pattern Recognition Workshops, 2012, pp. 20-27.

[135] X. Yang and Y. L. Tian, "Eigenjoints-based action recognition using naive-bayesnearest-neighbor," in IEEE Computer Society Conference on Computer Vision and Pattern Recognition Workshops, 2012, pp. 14-19.

[136] F. Ofli, R. Chaudhry, G. Kurillo, R. Vidal, and R. Bajcsy, "Sequence of the most informative joints (smij): A new representation for human skeletal action recognition," 
Journal of Visual Communication and Image Representation, vol. 25, no. 1, pp. 24-38, 2014.

[137] K. Yun, J. Honorio, D. Chattopadhyay, T. L. Berg, and D. Samaras, “Two-person interaction detection using body-pose features and multiple instance learning," in Computer Vision and Pattern Recognition Workshops (CVPRW), 2012 IEEE Computer Society Conference on. IEEE, 2012.

[138] Y. Du, Y. Fu, and L. Wang, "Representation learning of temporal dynamics for skeletonbased action recognition," IEEE Transactions on Image Processing, vol. 25, no. 7, pp. 3010-3022, 2016.

[139] Y. G. C. H. Ji, Y., "Interactive body part contrast mining for human interaction recognition," in ICMEW, 2014.

[140] W. Li, L. Wen, M. C. Chuah, and S. Lyu, "Category-blind human action recognition: A practical recognition system," in 2015 IEEE International Conference on Computer Vision (ICCV), 2015, pp. 4444-4452.

[141] T.-T. N. G. W. Amir Shahroudy, Jun Liu, "Ntu rgb+d: A large scale dataset for 3d human activity analysis," in IEEE Conference on Computer Vision and Pattern Recognition (CVPR), 2016.

[142] R. Vemulapalli, F. Arrate, and R. Chellappa, "Human action recognition by representing 3d skeletons as points in a lie group," in 2014 IEEE Conference on Computer Vision and Pattern Recognition, 2014, pp. 588-595.

[143] M. D. Zeiler and R. Fergus, "Visualizing and understanding convolutional networks," in European Conference on Computer Vision (ECCV). Springer, 2014, pp. 818-833. 